\title{
PRINCIPALIZATION ALGORITHM VIA CLASS GROUP STRUCTURE
}

\author{
DANIEL C. MAYER
}

\begin{abstract}
For an algebraic number field $K$ with 3-class group $\mathrm{Cl}_{3}(K)$ of type $(3,3)$, the structure of the 3-class groups $\mathrm{Cl}_{3}\left(N_{i}\right)$ of the four unramified cyclic cubic extension fields $N_{i}$, $1 \leq i \leq 4$, of $K$ is calculated with the aid of presentations for the metabelian Galois group $\mathrm{G}_{3}^{2}(K)=\mathrm{Gal}\left(\mathrm{F}_{3}^{2}(K) \mid K\right)$ of the second Hilbert 3-class field $\mathrm{F}_{3}^{2}(K)$ of $K$. In the case of a quadratic base field $K=\mathbb{Q}(\sqrt{D})$ it is shown that the structure of the 3-class groups of the four $S_{3}$-fields $N_{1}, \ldots, N_{4}$ frequently determines the type of principalization of the 3-class group of $K$ in $N_{1}, \ldots, N_{4}$. This provides an alternative to the classical principalization algorithm by Scholz and Taussky. The new algorithm, which is easily automatizable and executes very quickly, is implemented in PARI/GP and is applied to all 4596 quadratic fields $K$ with 3-class group of type $(3,3)$ and discriminant $-10^{6}<D<10^{7}$ to obtain extensive statistics of their principalization types and the distribution of their second 3-class groups $\mathrm{G}_{3}^{2}(K)$ on various coclass trees of the coclass graphs $\mathcal{G}(3, r), 1 \leq r \leq 6$, in the sense of Eick, Leedham-Green, and Newman.
\end{abstract}

\section{INTRODUCTION}

The principal ideal theorem, which has been conjectured by Hilbert in 1898 [19, p. 14], states that each ideal of a number field $K$ becomes principal when it is extended to the Hilbert class field $\mathrm{F}^{1}(K)$ of $K$, that is the maximal abelian unramified extension field of $K$. Inspired by the Artin-Furtwängler proof [2, 19] of the principal ideal theorem, Scholz and Taussky investigated the principalization in intermediate fields $K<N<\mathrm{F}_{3}^{1}(K)$ between a base field $K$ with 3-class group of type $(3,3)$ and its Hilbert 3-class field $\mathrm{F}_{3}^{1}(K)$. They developed an algorithm for computing the principalization of $K$ in its four unramified cyclic cubic extension fields $N_{1}, \ldots, N_{4}$ for the case of a complex quadratic base field $K$ [40]. This algorithm is probabilistic, since it decides whether an ideal $\mathfrak{a}$ of $K$ becomes principal in $N_{i}$, for some $1 \leq i \leq 4$, by testing local cubic residue characters of a principal ideal cube $(\alpha)=\mathfrak{a}^{3}$, associated with the ideal $\mathfrak{a}$, and of a fundamental unit $\varepsilon_{i}$ of the non-Galois cubic subfield $L_{i}$ of the complex $S_{3}$-field $N_{i}$ with respect to a series of rational test primes $\left(p_{\ell}\right)_{\ell \geq 1}$ and terminating when a critical test prime occurs [28, Algorithm, Step 8, p. 81]. An upper bound for the minimal critical test prime $p_{\ell_{0}}$ cannot be given effectively. $p_{\ell_{0}}$ can only be estimated by means of Chebotarëv's density theorem [40, 22, and thus causes uncertainty.

An entirely different approach to the principalization problem will be presented in this article. It is based on a purely group theoretical connection between the structure of the abelianizations $M_{i} / M_{i}^{\prime}$ of the four maximal normal subgroups $M_{i}$ of an arbitrary metabelian 3-group $G$ with abelianization $G / G^{\prime}$ of type $(3,3)$ and the kernels $\operatorname{ker}\left(\mathrm{T}_{i}\right)$ of the transfers $\mathrm{T}_{i}: G / G^{\prime} \longrightarrow M_{i} / M_{i}^{\prime}$, $1 \leq i \leq 4$. By the Artin reciprocity law of class field theory [1], a corresponding number theoretical connection is established between the structure of the 3-class groups $\mathrm{Cl}_{3}\left(N_{i}\right)$ of the four unramified cyclic cubic extension fields $N_{i}$ of an arbitrary algebraic number field $K$ with 3-class group $\mathrm{Cl}_{3}(K)$ of type $(3,3)$ and the principalization kernels $\operatorname{ker}\left(\mathrm{j}_{N_{i} \mid K}\right)$ of the class extension homomorphisms $\mathrm{j}_{N_{i} \mid K}: \mathrm{Cl}_{3}(K) \longrightarrow \mathrm{Cl}_{3}\left(N_{i}\right), 1 \leq i \leq 4$. The correspondence is obtained by applying the group theoretical statements about $G$ and $M_{1}, \ldots, M_{4}$ to the second 3-class group $\mathrm{G}_{3}^{2}(K)=\operatorname{Gal}\left(\mathrm{F}_{3}^{2}(K) \mid K\right)$ of $K$ [30, that is the Galois group of the second Hilbert 3-class field $\mathrm{F}_{3}^{2}(K)=\mathrm{F}_{3}^{1}\left(\mathrm{~F}_{3}^{1}(K)\right)$ of $K$,

Date: April 26, 2013.

2000 Mathematics Subject Classification. Primary 11R29, 11R11, 11R16, 11R20; Secondary $20 \mathrm{D} 15$.

Key words and phrases. 3-class groups, principalization of 3-classes, quadratic fields, $S_{3}$-fields, metabelian 3groups, coclass graphs.

Research supported by the Austrian Science Fund, Grant Nr. J0497-PHY. 
and its maximal subgroups $\operatorname{Gal}\left(\mathrm{F}_{3}^{2}(K) \mid N_{i}\right), 1 \leq i \leq 4$. We call the family $\tau(G)=\left(M_{i} / M_{i}^{\prime}\right)_{1 \leq i \leq 4}$ the transfer target type (TTT) of $G$ and the family $\varkappa(G)=\left(\operatorname{ker}\left(\mathrm{T}_{i}\right)\right)_{1 \leq i \leq 4}$ the transfer kernel type (TKT) of $G$ (briefly called transfer type in [30, § 2.2, p. 476]).

We begin by comparing the four little two-stage towers $K<\mathrm{F}_{3}^{1}(K)<\mathrm{F}_{3}^{1}\left(N_{i}\right), 1 \leq i \leq 4$, and the big two-stage tower $K<\mathrm{F}_{3}^{1}(K)<\mathrm{F}_{3}^{2}(K)$ of 3-class fields in $\S$ 2. Based on these relationships, $\S 3$ is devoted to proving that the 3-class groups $\mathrm{Cl}_{3}\left(N_{i}\right), 1 \leq i \leq 4$, have the structure of nearly homocyclic abelian 3-groups of 3-rank two, provided the index of nilpotency of the second 3-class group $G=\mathrm{G}_{3}^{2}(K)=\operatorname{Gal}\left(\mathrm{F}_{3}^{2}(K) \mid K\right)$ is not too small. The structure of the remaining 3-class groups, which are partially of 3-rank three as predicted at the beginning of $\S$ 田 is determined in $\S 4.1$ if $G$ is of coclass 1 . The central results of this paper concern groups $G$ of $\operatorname{coclass} \operatorname{cc}(G) \geq 2$ and are developed successively, beginning in $\S 4.2$ on the general method of proof, for sporadic groups of coclass 2 with bicyclic centre in $\S 4.3$ and with cyclic centre in $\S 4.4$, for groups of coclass 2 on coclass trees [16] in $\S$ 4.5, and finally, for all groups of coclass $\operatorname{cc}(G) \geq 3$ in $\S 4.6$.

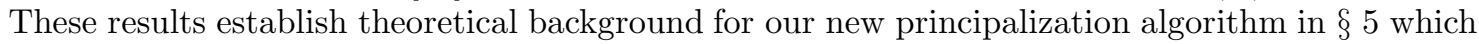
is based on the invariant $\varepsilon=\varepsilon(K)$, the number of elementary abelian 3-groups of type $(3,3,3)$ among the 3 -class groups $\mathrm{Cl}_{3}\left(N_{i}\right), 1 \leq i \leq 4$. In view of future generalization 34 to base fields $K$ with $\mathrm{Cl}_{3}(K)$ of type $(9,3)$, we can also define $\varepsilon$ as the number of 3 -class groups of elevated 3 -rank at least three. As opposed to Scholz and Taussky's classical algorithm for determining the 3 -principalization over complex quadratic fields [40, $\S 1$, pp. 20-31], which is also described in [28, Algorithm, pp. 80-83] and in [11, § 3, pp. 9-29, and Appendix A, pp. 96-113], and is extended to the $p$-principalization with an odd prime $p$ over arbitrary quadratic fields in $[22, \S 1$, pp. $3-6$, and $\S 3-4$, pp. 12-24], the new 3-principalization algorithm for arbitrary quadratic fields is easily automatizable and executes very quickly. In $\S 6$ an extensive application of our principalization algorithm via class group structure is presented.

\section{LitTle AND BIG TWO-STAGE TOWERS OF 3-CLASS FIELDS}

Let $K$ be an algebraic number field with 3 -class group $\mathrm{Cl}_{3}(K)$ of type $(3,3)$. By class field theory [32, Cor. 3.1, p. 838], there exist four unramified cyclic cubic extension fields $N_{1}, \ldots, N_{4}$ of $K$ within the first Hilbert 3-class field $\mathrm{F}_{3}^{1}(K)$ of $K$. Consequently, the first Hilbert 3-class field $\mathrm{F}_{3}^{1}\left(N_{i}\right)$ of $N_{i}$ is an intermediate field between $\mathrm{F}_{3}^{1}(K)$ and the second Hilbert 3-class field $\mathrm{F}_{3}^{2}(K)$ of $K$, for each $1 \leq i \leq 4$.

By $\Gamma_{i}=\operatorname{Gal}\left(\mathrm{F}_{3}^{1}\left(N_{i}\right) \mid K\right)$ we denote the Galois groups of the four little two-stage towers of $K$, $K<\mathrm{F}_{3}^{1}(K) \leq \mathrm{F}_{3}^{1}\left(N_{i}\right)$, where $1 \leq i \leq 4$, and by $G=\operatorname{Gal}\left(\mathrm{F}_{3}^{2}(K) \mid K\right)$ the Galois group of the big two-stage tower of $K, K<\mathrm{F}_{3}^{1}(K) \leq \mathrm{F}_{3}^{2}(K)$.

Proposition 2.1. $G$ and $\Gamma_{i}$ are metabelian 3-groups with abelianizations $G / G^{\prime}$ and $\Gamma_{i} / \Gamma_{i}^{\prime}$ of type $(3,3)$. They are non-abelian, except for a single-stage tower $\mathrm{F}_{3}^{2}(K)=\mathrm{F}_{3}^{1}\left(N_{i}\right)=\mathrm{F}_{3}^{1}(K)$.

$G$ contains four maximal normal subgroups $M_{1}, \ldots, M_{4}$ and each $\Gamma_{i}$ contains an abelian maximal subgroup $A_{i}=\operatorname{Gal}\left(\mathrm{F}_{3}^{1}\left(N_{i}\right) \mid N_{i}\right)$ isomorphic to the 3 -class group $\mathrm{Cl}_{3}\left(N_{i}\right)$ of $N_{i}$, for $1 \leq i \leq 4$.

The connection between $\Gamma_{i}$ and $G$, resp. between $A_{i}$ and $M_{i}$, for $1 \leq i \leq 4$, is given by

$$
\begin{aligned}
\Gamma_{i} & \simeq G / M_{i}^{\prime}, \\
A_{i} & \simeq M_{i} / M_{i}^{\prime} .
\end{aligned}
$$

Proof. Since $\mathrm{F}_{3}^{1}(K)$ is the maximal abelian unramified extension field of $K$ with a power of 3 as relative degree, the Galois groups $G=\operatorname{Gal}\left(\mathrm{F}_{3}^{2}(K) \mid K\right)$ and $\Gamma_{i}=\operatorname{Gal}\left(\mathrm{F}_{3}^{1}\left(N_{i}\right) \mid K\right), 1 \leq i \leq 4$, are non-abelian, except in the degenerate case $\mathrm{F}_{3}^{2}(K)=\mathrm{F}_{3}^{1}\left(N_{i}\right)=\mathrm{F}_{3}^{1}(K)$. That $\Gamma_{i}$ is also non-abelian when $G$ is non-abelian follows from the relation $\Gamma_{i} \simeq G / M_{i}^{\prime}$, which is shown at the end of this proof, together with Cor. 3.1 and Cor. 3.2 in [30, pp. 476 and 480], where it is proved that $M_{i}^{\prime}$ is contained in the third member of the lower central series of $G$ and thus strictly smaller than $G^{\prime}$, for each $1 \leq i \leq 4$. The abelian commutator subgroups of $G$ and $\Gamma_{i}$ are given by

$$
G^{\prime}=\operatorname{Gal}\left(\mathrm{F}_{3}^{2}(K) \mid \mathrm{F}_{3}^{1}(K)\right) \simeq \mathrm{Cl}_{3}\left(\mathrm{~F}_{3}^{1}(K)\right)
$$

and

$$
\Gamma_{i}^{\prime}=\operatorname{Gal}\left(\mathrm{F}_{3}^{1}\left(N_{i}\right) \mid \mathrm{F}_{3}^{1}(K)\right)<A_{i}=\operatorname{Gal}\left(\mathrm{F}_{3}^{1}\left(N_{i}\right) \mid N_{i}\right) \simeq \mathrm{Cl}_{3}\left(N_{i}\right),
$$


by the Artin reciprocity law 1 . Hence, $G$ and $\Gamma_{i}$ are metabelian 3 -groups with abelianizations $G / G^{\prime}$ and $\Gamma_{i} / \Gamma_{i}^{\prime}$ isomorphic to $\operatorname{Gal}\left(\mathrm{F}_{3}^{1}(K) \mid K\right) \simeq \mathrm{Cl}_{3}(K)$ and thus of type $(3,3)$.

Since the four maximal normal subgroups $M_{1}, \ldots, M_{4}$ of $G$ are associated with the extensions $N_{1}, \ldots, N_{4}$ by $M_{i}=\operatorname{Gal}\left(\mathrm{F}_{3}^{2}(K) \mid N_{i}\right)$, their commutator subgroups are given by

$$
M_{i}^{\prime}=\operatorname{Gal}\left(\mathrm{F}_{3}^{2}(K) \mid \mathrm{F}_{3}^{1}\left(N_{i}\right)\right)
$$

and their abelianizations by

$$
M_{i} / M_{i}^{\prime}=\operatorname{Gal}\left(\mathrm{F}_{3}^{2}(K) \mid N_{i}\right) / \operatorname{Gal}\left(\mathrm{F}_{3}^{2}(K) \mid \mathrm{F}_{3}^{1}\left(N_{i}\right)\right) \simeq A_{i}=\operatorname{Gal}\left(\mathrm{F}_{3}^{1}\left(N_{i}\right) \mid N_{i}\right) \simeq \mathrm{Cl}_{3}\left(N_{i}\right) .
$$

Since $M_{i}^{\prime}$ is a characteristic subgroup of $M_{i}$, it is a normal subgroup of $G$ and we have the relation

$$
G / M_{i}^{\prime}=\operatorname{Gal}\left(\mathrm{F}_{3}^{2}(K) \mid K\right) / \operatorname{Gal}\left(\mathrm{F}_{3}^{2}(K) \mid \mathrm{F}_{3}^{1}\left(N_{i}\right)\right) \simeq \operatorname{Gal}\left(\mathrm{F}_{3}^{1}\left(N_{i}\right) \mid K\right)=\Gamma_{i} .
$$

In the following $\S$, we determine the standard structure of the abelian maximal normal subgroups $A_{i} \simeq \mathrm{Cl}_{3}\left(N_{i}\right)$ of $\Gamma_{i}, 1 \leq i \leq 4$, for a given second 3-class group $G$ of $K$.

\section{NeARly homocyclic 3-Class groups of 3-RANK TWO}

The concept of a nearly homocyclic abelian $p$-group with an arbitrary prime $p \geq 2$ appears in [9, Thm. 3.4, p. 68] (see our Appendix) and is treated systematically in [36, § 2.4]. For our purpose, it suffices to consider the special case $p=3$.

Definition 3.1. By the nearly homocyclic abelian 3-group $\mathrm{A}(3, n)$ of order $3^{n}$, for an integer $n \geq 2$, we understand the abelian group of type $\left(3^{q+r}, 3^{q}\right)$, where $n=2 q+r$ with integers $q \geq 1$ and $0 \leq r<2$. Additionally, including two degenerate cases, we define that $\mathrm{A}(3,1)$ denotes the cyclic group $C_{3}$ of order 3 and $\mathrm{A}(3,0)$ the trivial group 1.

The application of Blackburn's well-known Theorem 3.4 in [9, p. 68] to the Galois groups $\Gamma_{i}=\operatorname{Gal}\left(\mathrm{F}_{3}^{1}\left(N_{i}\right) \mid K\right)$ of the four little two-stage towers $K<\mathrm{F}_{3}^{1}(K) \leq \mathrm{F}_{3}^{1}\left(N_{i}\right)$ with abelian maximal normal subgroups $A_{i}=\operatorname{Gal}\left(\mathrm{F}_{3}^{1}\left(N_{i}\right) \mid N_{i}\right) \simeq \mathrm{Cl}_{3}\left(N_{i}\right)$ will show that, in general, the 3-class groups $\mathrm{Cl}_{3}\left(N_{i}\right), 1 \leq i \leq 4$, are nearly homocyclic abelian 3 -groups $\mathrm{A}(3, u+v)$ of type $\left(3^{u}, 3^{v}\right)$ with $1 \leq v \leq u \leq v+1$. The phrase 'in general' is made precise in the following theorems, where we distinguish second 3-class groups $G=\operatorname{Gal}\left(\mathrm{F}_{3}^{2}(K) \mid K\right)$ of coclass cc $(G)=1 \mathrm{in} \S 3.1$ and of coclass $\operatorname{cc}(G) \geq 2$ in $\S 3.2$, and use concepts and notation of our papers [30, 31, as recalled in the sequel.

3.1. Second 3-class groups $G$ of coclass $\operatorname{cc}(G)=1$. Let $G$ be a metabelian 3-group of order $|G|=3^{n}$ and nilpotency class $\operatorname{cl}(G)=m-1$, where $n=m \geq 3$. Then $G$ is of $\operatorname{coclass} \operatorname{cc}(G)=$ $n-\operatorname{cl}(G)=1$ and the commutator factor group $G / G^{\prime}$ of $G$ is of type $(3,3)$, 935. The lower central series of $G$ is defined recursively by $\gamma_{1}(G)=G$ and $\gamma_{j}(G)=\left[\gamma_{j-1}(G), G\right]$ for $j \geq 2$. In particular, $\gamma_{2}(G)=[G, G]=G^{\prime}$ denotes the commutator subgroup.

The centralizer $\chi_{2}(G)=\left\{g \in G \mid[g, u] \in \gamma_{4}(G)\right.$ for all $\left.u \in \gamma_{2}(G)\right\}$ of the two-step factor group $\gamma_{2}(G) / \gamma_{4}(G)$, that is,

$$
\chi_{2}(G) / \gamma_{4}(G)=\text { Centralizer }_{G / \gamma_{4}(G)}\left(\gamma_{2}(G) / \gamma_{4}(G)\right),
$$

is the biggest subgroup of $G$ such that $\left[\chi_{2}(G), \gamma_{2}(G)\right] \leq \gamma_{4}(G)$. It is characteristic, contains the commutator group $\gamma_{2}(G)$, and coincides with $G$ if and only if $m=3$. Let the isomorphism invariant $k=k(G)$, the defect of commutativity of $G$, be defined by

$$
\left[\chi_{2}(G), \gamma_{2}(G)\right]=\gamma_{m-k}(G),
$$

where $k=0$ for $3 \leq m \leq 4$, and $0 \leq k \leq 1$ for $m \geq 5$, according to Miech [35. p. 331].

Suppose that generators of $G=\langle x, y\rangle$ are selected such that $x \in G \backslash \chi_{2}(G)$, if $m \geq 4$, and $y \in \chi_{2}(G) \backslash \gamma_{2}(G)$. We define the main commutator $s_{2}=[y, x] \in \gamma_{2}(G)$ and the higher commutators $s_{j}=\left[s_{j-1}, x\right]=s_{j-1}^{x-1} \in \gamma_{j}(G)$ for $j \geq 3$. Then $G$ satisfies two relations for third powers of the generators $x$ and $y$ of $G$,

$$
x^{3}=s_{m-1}^{w} \quad \text { and } \quad y^{3} s_{2}^{3} s_{3}=s_{m-1}^{z} \quad \text { with exponents } \quad-1 \leq w, z \leq 1,
$$


according to Miech [35, Thm. 2, (3), p. 332]. Blackburn uses the notation $\delta=w$ and $\gamma=z$ for these relational parameters [9, (36)-(37), p. 84].

Additionally, the group $G$ satisfies relations for third powers of higher commutators,

$$
s_{j+1}^{3} s_{j+2}^{3} s_{j+3}=1 \quad \text { for } 1 \leq j \leq m-2,
$$

and the main commutator relation of Miech [35, Thm. 2, (2), p. 332],

$$
\left[y, s_{2}\right]=s_{m-1}^{a} \in\left[\chi_{2}(G), \gamma_{2}(G)\right]=\gamma_{m-k}(G),
$$

with exponent $-1 \leq a \leq 1$. Blackburn uses the notation $\beta=a$ [9, (33), p. 82].

By $G_{a}^{m}(z, w)$ we denote the representative of an isomorphism class of metabelian 3-groups $G$ of coclass $\operatorname{cc}(G)=1$ and of order $|G|=3^{m}$, which satisfies the relations (3) and (2) with a fixed system of parameters $a, w$, and $z$. Obviously, the defect is $k=0$ if and only if $a=0$.

The maximal normal subgroups $M_{i}$ of $G$ contain the commutator group $\gamma_{2}(G)$ of $G$ as a normal subgroup of index 3 and thus are of the shape $M_{i}=\left\langle g_{i}, \gamma_{2}(G)\right\rangle$. We define a fixed order by $g_{1}=y$, $g_{2}=x, g_{3}=x y$ and $g_{4}=x y^{-1}$. The commutator subgroups $\gamma_{2}\left(M_{i}\right)$ are of the general form $\gamma_{2}\left(M_{i}\right)=\left\langle s_{2}, \ldots, s_{m-1}\right\rangle^{g_{i}-1}$, according to [30, Cor. 3.1, p. 476], and in particular

$$
\begin{aligned}
\gamma_{2}\left(M_{1}\right) & = \begin{cases}1, & \text { if } k=0 \\
\gamma_{m-1}(G), & \text { if } k=1\end{cases} \\
\gamma_{2}\left(M_{i}\right) & =\gamma_{3}(G) \text { for } 2 \leq i \leq 4
\end{aligned}
$$

Theorem 3.1. (Transfer target type $\tau(G)$ for groups $G$ with $\operatorname{cc}(G)=1, \operatorname{cl}(G) \geq 5$ )

The structure of the 3-class groups $\mathrm{Cl}_{3}\left(N_{i}\right)$ of the four unramified cyclic cubic extension fields $N_{1}, \ldots, N_{4}$ of an arbitrary base field $K$ having a 3 -class group $\mathrm{Cl}_{3}(K)$ of type $(3,3)$ and a second 3 -class group $G=\operatorname{Gal}\left(\mathrm{F}_{3}^{2}(K) \mid K\right)$ of coclass $\operatorname{cc}(G)=1$, order $|G|=3^{n}$, and class $\operatorname{cl}(G)=m-1$, where $m=n \geq 3$, is given by the following nearly homocyclic abelian 3-groups.

$$
\begin{aligned}
& \mathrm{Cl}_{3}\left(N_{1}\right) \simeq \begin{cases}\mathrm{A}(3, m-1), & \text { if }\left[\chi_{2}(G), \gamma_{2}(G)\right]=1, k=0, m \geq 5, \\
\mathrm{~A}(3, m-2), & \text { if }\left[\chi_{2}(G), \gamma_{2}(G)\right]=\gamma_{m-1}(G), k=1, m \geq 6,\end{cases} \\
& \mathrm{Cl}_{3}\left(N_{i}\right) \simeq \mathrm{A}(3,2) \text { for } 2 \leq i \leq 4, \text { if } m \geq 4 .
\end{aligned}
$$

Proof. The metabelian 3-groups $\Gamma_{i}=\operatorname{Gal}\left(\mathrm{F}_{3}^{1}\left(N_{i}\right) \mid K\right)$, having an abelian maximal subgroup $A_{i} \simeq$ $\mathrm{Cl}_{3}\left(N_{i}\right)$, are of coclass $\operatorname{cc}(G)=1$, according to Heider and Schmithals [22, Kor., p. 9] or also to 30. Cor. 3.1, p. 476, and Cor. 3.2, p. 480], since we are dealing with 3-groups here. This is the crucial condition for the applicability of Blackburn's Theorem 3.4 [9, p. 68] (see our Appendix). It is also the reason why we need the connection between $G$ and $\Gamma_{1}, \ldots, \Gamma_{4}$.

We begin by investigating the distinguished extension $N_{1}$.

Suppose first that $\left[\chi_{2}(G), \gamma_{2}(G)\right]=1$, that is $k=0$. Then $\gamma_{2}\left(M_{1}\right)=1$, by [30, Cor. 3.1], the group $\Gamma_{1}$ is isomorphic to $G$, by formula (1), and has the order $\left|\Gamma_{1}\right|=3^{m}$. For $m \geq 5$, the abelian normal subgroup $A_{1}$ of $\Gamma_{1}$ is isomorphic to $\mathrm{A}(3, m-1)$, according to Blackburn [9, Thm. 3.4], where the lower bound $p+2=5$ for the index of nilpotency $m$ is due to the specialisation $p=3$.

Next we consider the case $\left[\chi_{2}(G), \gamma_{2}(G)\right]=\gamma_{m-1}(G)$, that is $k=1$. Then $\gamma_{2}\left(M_{1}\right)=\gamma_{m-1}(G)$, by [30. Cor. 3.1], the group $\Gamma_{1}$ is isomorphic to $G / \gamma_{m-1}(G)$, by formula (11), and is therefore the immediate predecessor of $G$ [36, p. 182] on the coclass graph $\mathcal{G}(3,1)$ in Figure 1, thus being isomorphic to the mainline group $G_{0}^{m-1}(0,0)$ and of order $\left|\Gamma_{1}\right|=3^{m-1}$. For $m \geq 6$, it follows that $A_{1} \simeq \mathrm{A}(3, m-2)$, according to 9 , Thm. 3.4].

However, Blackburn's result cannot be applied to the other three extensions $N_{i}$ with $2 \leq i \leq 4$, since the three isomorphic groups $\Gamma_{i} \simeq G / \gamma_{3}(G)$ [30, Cor. 3.1] are of order $\left|\Gamma_{i}\right|=3^{\mu}$ with exponent $\mu=3<5$. Therefore we must determine the structure of the abelian normal subgroup $A_{i}$ of $\Gamma_{i}$ by the following consideration. In the case $m \geq 4$, the group $\gamma_{3}(G)>1$ is non-trivial. Since $\Gamma_{i} \simeq G / \gamma_{3}(G)$ is a predecessor of $G$ [36, p. 182] on the coclass graph $\mathcal{G}(3,1)$ in Figure 1] it can only be isomorphic to the extra special 3-group $G_{0}^{3}(0,0)$ of exponent 3 on the mainline, whose four maximal normal subgroups are all abelian of type $(3,3)$ and are thus isomorphic to $\mathrm{A}(3,2)$. 
The vertices of the coclass graph $\mathcal{G}(3,1)$ in Figure 1 represent all isomorphism classes of finite 3 -groups $G$ with coclass cc $(G)=1$. Two vertices are connected by a directed edge $H \rightarrow G$ if $G$ is isomorphic to the last lower central quotient $H / \gamma_{c}(H)$ where $c=\operatorname{cl}(H)$ denotes the nilpotency class of $H$, and $|H|=3|G|$, i. e. $\gamma_{c}(H)$ is cyclic of order 3 . The graph $\mathcal{G}(3,1)$ has also been drawn in [4, Fig. 4.3, p. 63], [26, pp. 194-195], [36, p. 189 f.], [12, p. 46], [14, § 9].

The two top vertices (contour squares) are abelian. $C_{9}$ is isolated and $C_{3} \times C_{3}$ is the root of the unique coclass tree $\mathcal{T}\left(C_{3} \times C_{3}\right)$ of $\mathcal{G}(3,1)$. All other vertices (full discs) are metabelian, according to Blackburn [10, Thm. 6, p. 26]. Groups with defect $k=0$ are represented by bigger discs than those with $k=1$. Numbers in angles denote the identifiers of groups in the SmallGroup library [8] and in GAP 4.4 [20, where we omit the orders, which are given on the left hand scale.

FIgURE 1. Root $C_{3} \times C_{3}$ and branches $\mathcal{B}(j), 2 \leq j \leq 7$, on the coclass graph $\mathcal{G}(3,1)$

Order $3^{m}$

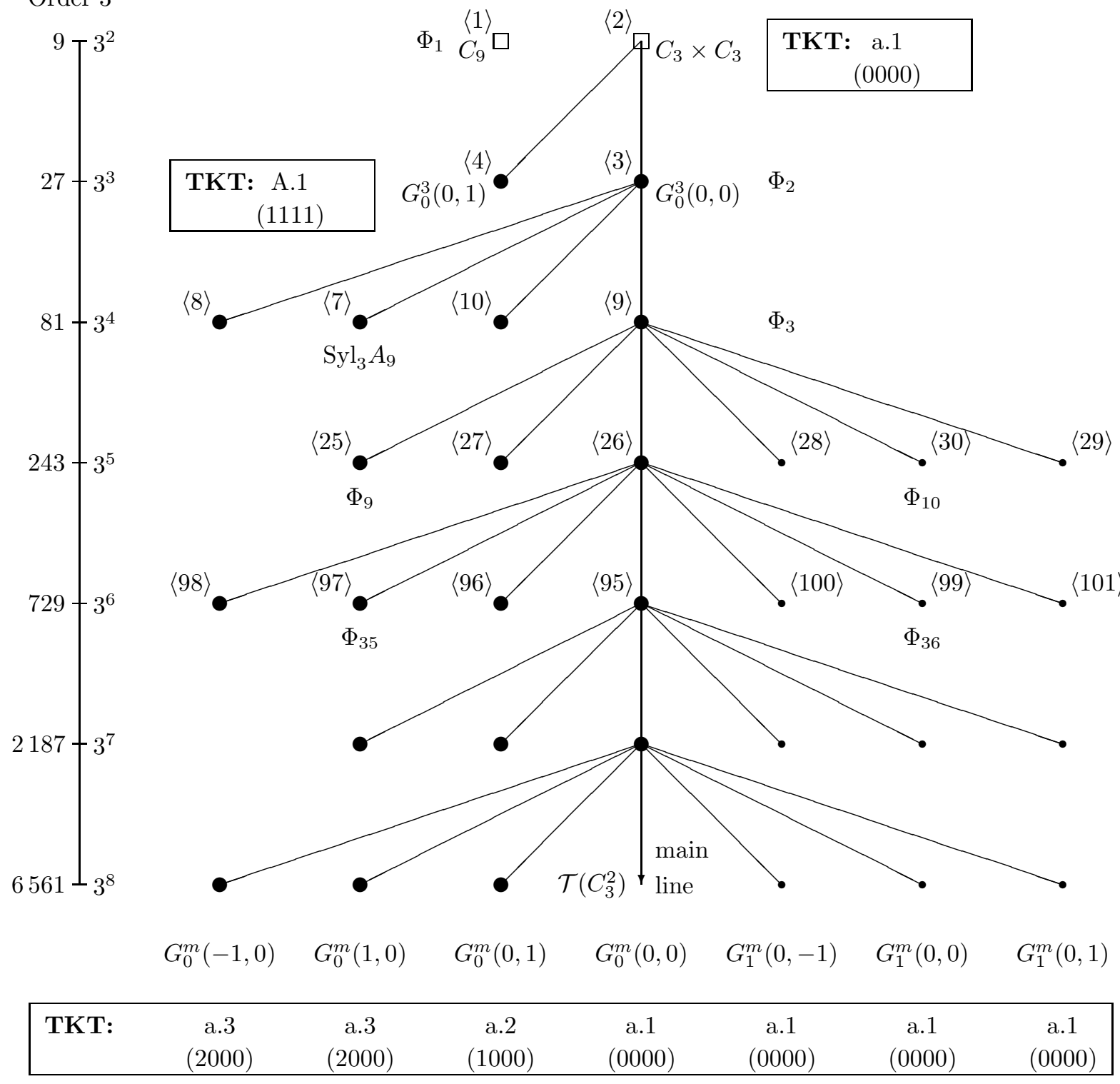

The symbols $\Phi_{s}$ denote isoclinism families $[21,13,23$. The principalization types or transfer kernel types, briefly TKT, 31, Thm. 2.5, p. 478, and Tbl. 6-7, pp. 492-493] in the bottom 
rectangle concern all vertices located vertically above, except indicated otherwise. Periodicity of length 2 [15, p. 275], $\mathcal{B}(j) \simeq \mathcal{B}(j+2)$ for $j \geq 4$, sets in with branch $\mathcal{B}(4)$, having root of order $3^{4}$.

For $G$ of coclass $\operatorname{cc}(G)=1$, it remains to investigate the structure of the following 3-class groups:

- $\mathrm{Cl}_{3}\left(N_{1}\right)$ of order $3^{m-1}=3^{3}$ for $m=4$ (where $\left[\chi_{2}(G), \gamma_{2}(G)\right]=1, k=0$ ),

- $\mathrm{Cl}_{3}\left(N_{1}\right)$ of order $3^{m-2}=3^{3}$ for $m=5$ and $\left[\chi_{2}(G), \gamma_{2}(G)\right]=\gamma_{4}(G)>1, k=1$,

- all four $\mathrm{Cl}_{3}\left(N_{i}\right)$ with $1 \leq i \leq 4$ of order $3^{2}$ for $m=3$ (where $k=0$ ).

3.2. Second 3-class groups $G$ of coclass $\operatorname{cc}(G) \geq 2$. Metabelian 3-groups $G$ of $\operatorname{coclass} \operatorname{cc}(G) \geq$ 2 must have at least one bicyclic factor $\gamma_{3}(G) / \gamma_{4}(G)$. Similarly as in $\S 2$ of [30], we declare an isomorphism invariant $e=e(G)$ of $G$ by $e+1=\min \left\{3 \leq j \leq m|1 \leq| \gamma_{j}(G) / \gamma_{j+1}(G) \mid \leq 3\right\}$. This invariant $2 \leq e \leq m-1$ characterizes the first cyclic factor $\gamma_{e+1}(G) / \gamma_{e+2}(G)$ of the lower central series of $G$, except $\gamma_{2}(G) / \gamma_{3}(G)$, which is always cyclic. We can calculate $e$ from the 3 -exponent $n$ of the order $|G|=3^{n}$ and the class $\operatorname{cl}(G)=m-1$, resp. the index $m$ of nilpotency, of $G$ by the formula $e=n-m+2$. Since the coclass of $G$ is given by $\operatorname{cc}(G)=n-\operatorname{cl}(G)=n-m+1$, we have the relation $e=\operatorname{cc}(G)+1$.

For a group $G$ of coclass $\operatorname{cc}(G) \geq 2$ we need a generalization of the group $\chi_{2}(G)$. Denoting by $m$ the index of nilpotency of $G$, we let $\chi_{j}(G)$ with $2 \leq j \leq m-1$ be the centralizers of two-step factor groups $\gamma_{j}(G) / \gamma_{j+2}(G)$ of the lower central series, that is, the biggest subgroups of $G$ with the property $\left[\chi_{j}(G), \gamma_{j}(G)\right] \leq \gamma_{j+2}(G)$. They form an ascending chain of characteristic subgroups of $G, \gamma_{2}(G) \leq \chi_{2}(G) \leq \ldots \leq \chi_{m-2}(G)<\chi_{m-1}(G)=G$, which contain the commutator subgroup $\gamma_{2}(G)$, and $\chi_{j}(G)$ coincides with $G$ if and only if $j \geq m-1$. Similarly as in $\S 2$ of [30, we characterize the smallest two-step centralizer different from the derived subgroup by an isomorphism invariant $s=s(G)=\min \left\{2 \leq j \leq m-1 \mid \chi_{j}(G)>\gamma_{2}(G)\right\}$.

The following assumptions for a metabelian 3-group $G$ of $\operatorname{coclass} \operatorname{cc}(G) \geq 2$ with abelianization $G / \gamma_{2}(G)$ of type $(3,3)$ can always be satisfied, according to [36. Satz 3.4.5, p. 94] (see our appendix).

Let $G$ be a metabelian 3-group of coclass $\operatorname{cc}(G) \geq 2$ with abelianization $G / \gamma_{2}(G)$ of type $(3,3)$. Assume that $G$ has order $|G|=3^{n}$, class $\operatorname{cl}(G)=m-1$, and invariant $e=n-m+2 \geq 3$, where $4 \leq m<n \leq 2 m-3$. Let generators of $G=\langle x, y\rangle$ be selected such that $\gamma_{3}(G)=\left\langle y^{3}, x^{3}, \gamma_{4}(G)\right\rangle$, $x \in G \backslash \chi_{s}(G)$ if $s<m-1$, and $y \in \chi_{s}(G) \backslash \gamma_{2}(G)$. Suppose that a fixed order of the four maximal normal subgroups of $G$ is defined by $M_{i}=\left\langle g_{i}, \gamma_{2}(G)\right\rangle$ with $g_{1}=y, g_{2}=x, g_{3}=x y$, and $g_{4}=x y^{-1}$. Let the main commutator of $G$ be declared by $s_{2}=t_{2}=[y, x] \in \gamma_{2}(G)$ and higher commutators recursively by $s_{j}=\left[s_{j-1}, x\right], t_{j}=\left[t_{j-1}, y\right] \in \gamma_{j}(G)$ for $j \geq 3$. Starting with the powers $\sigma_{3}=y^{3}, \tau_{3}=x^{3} \in \gamma_{3}(G)$, let $\sigma_{j}=\left[\sigma_{j-1}, x\right], \tau_{j}=\left[\tau_{j-1}, y\right] \in \gamma_{j}(G)$ for $j \geq 4$. With exponents $-1 \leq \alpha, \beta, \gamma, \delta, \rho \leq 1$ as parameters, let the following relations be satisfied

$$
s_{2}^{3}=\sigma_{4} \sigma_{m-1}^{-\rho \beta} \tau_{4}^{-1}, \quad s_{3} \sigma_{3} \sigma_{4}=\sigma_{m-2}^{\rho \beta} \sigma_{m-1}^{\gamma} \tau_{e}^{\delta}, \quad t_{3}^{-1} \tau_{3} \tau_{4}=\sigma_{m-2}^{\rho \delta} \sigma_{m-1}^{\alpha} \tau_{e}^{\beta}, \quad \tau_{e+1}=\sigma_{m-1}^{-\rho} .
$$

Finally, let $\left[\chi_{s}(G), \gamma_{e}(G)\right]=\gamma_{m-k}(G)$ with the defect of commutativity $0 \leq k=k(G) \leq 1$ of $G$. Then, the defect is $k=0$ if and only if $\rho=0$.

By $G_{\rho}^{m, n}(\alpha, \beta, \gamma, \delta)$ we denote the representative of an isomorphism class of metabelian 3-groups $G$ with $G / \gamma_{2}(G)$ of type $(3,3)$, of coclass $\operatorname{cc}(G)=n-m+1 \geq 2$, class $\operatorname{cl}(G)=m-1$, and order $|G|=3^{n}$, which satisfies the relations (7) with a fixed system of parameters $(\alpha, \beta, \gamma, \delta, \rho)$.

Theorem 3.2. (Incomplete TTT $\left(\tau_{1}(G), \tau_{2}(G)\right)$ for groups $G$ with $\operatorname{cc}(G) \geq 3, \operatorname{cl}(G) \geq 5$ )

The structure of the 3 -class groups $\mathrm{Cl}_{3}\left(N_{i}\right)$ of the four unramified cyclic cubic extension fields $N_{1}, \ldots, N_{4}$ of an arbitrary base field $K$ having a 3 -class group $\mathrm{Cl}_{3}(K)$ of type $(3,3)$ and a second 3 -class group $G=\operatorname{Gal}\left(\mathrm{F}_{3}^{2}(K) \mid K\right)$ of coclass $\operatorname{cc}(G) \geq 2$, order $|G|=3^{n}$, class $\operatorname{cl}(G)=m-1$, and invariant $e=n-m+2 \geq 3$, where $4 \leq m<n \leq 2 m-3$, is given by the following nearly homocyclic abelian 3-groups. 


$$
\begin{aligned}
& \mathrm{Cl}_{3}\left(N_{1}\right) \simeq\left\{\begin{array}{l}
\mathrm{A}(3, m-1), \quad \text { if }\left[\chi_{s}(G), \gamma_{e}(G)\right]=1, k=0, m \geq 5, \\
\mathrm{~A}(3, m-2), \quad \text { if }\left[\chi_{s}(G), \gamma_{e}(G)\right]=\gamma_{m-1}(G), k=1, m \geq 6,
\end{array}\right. \\
& \mathrm{Cl}_{3}\left(N_{2}\right) \simeq \mathrm{A}(3, e) \text { for } e \geq 4, \\
& \mathrm{Cl}_{3}\left(N_{i}\right) \simeq \mathrm{A}(3,3) \text { for } 3 \leq i \leq 4, \text { if } \Gamma_{i} \not G_{0}^{4}(1,0) \simeq \mathrm{Syl}_{3} A_{9} .
\end{aligned}
$$

Proof. For each $1 \leq i \leq 4$, equation (1) specifies the order of the Galois group of the $i$ th little two-stage tower as $\left|\Gamma_{i}\right|=|G| /\left|\gamma_{2}\left(M_{i}\right)\right|$, where $|G|=3^{n}$ by assumption. According to [30, Cor. 3.2 , p. 480], the orders of the commutator subgroups of the $M_{i}$ are

$$
\left|\gamma_{2}\left(M_{i}\right)\right|= \begin{cases}3^{e-2} & \text { for } i=1,\left[\chi_{s}(G), \gamma_{e}(G)\right]=1, k=0, \\ 3^{e-1} & \text { for } i=1,\left[\chi_{s}(G), \gamma_{e}(G)\right]=\gamma_{m-1}(G), k=1, \\ 3^{m-3} & \text { for } i=2, \\ 3^{n-4} & \text { for } 3 \leq i \leq 4\end{cases}
$$

Using the relation $e=n-m+2$, we obtain

$$
\left|\Gamma_{i}\right|= \begin{cases}3^{m} & \text { for } i=1,\left[\chi_{s}(G), \gamma_{e}(G)\right]=1 \\ 3^{m-1} & \text { for } i=1, \quad\left[\chi_{s}(G), \gamma_{e}(G)\right]=\gamma_{m-1}(G), \\ 3^{e+1} & \text { for } i=2 \\ 3^{4} & \text { for } 3 \leq i \leq 4\end{cases}
$$

Since $\Gamma_{i}$, having an abelian maximal subgroup $A_{i} \simeq \mathrm{Cl}_{3}\left(N_{i}\right)$, is a metabelian 3-group of coclass $\operatorname{cc}(G)=1$ by [22, Kor., p. 9] or also by [30, Cor. 3.1-3.2, pp. 476 and 480], the structure of the abelian maximal normal subgroup $A_{i}$ of $\Gamma_{i}$ is given by

$$
\begin{aligned}
& A_{1} \simeq\left\{\begin{array}{l}
\mathrm{A}(3, m-1), \quad \text { if }\left[\chi_{s}(G), \gamma_{e}(G)\right]=1, k=0, m \geq 5, \\
\mathrm{~A}(3, m-2), \quad \text { if }\left[\chi_{s}(G), \gamma_{e}(G)\right]=\gamma_{m-1}(G), k=1, m-1 \geq 5,
\end{array}\right. \\
& A_{2} \simeq \mathrm{A}(3, e), \text { for } e+1 \geq 5, \\
& A_{i} \simeq \mathrm{A}(3,3) \text { for } 3 \leq i \leq 4, \text { if } \Gamma_{i} \not G_{0}^{4}(1,0) \simeq \operatorname{Syl}_{3} A_{9},
\end{aligned}
$$

according to [9, Thm. 3.4], for $1 \leq i \leq 2$, and by an immediate analysis of the four isomorphism classes of metabelian 3 -groups of order $3^{m}$ with index of nilpotency $m=4$, for $3 \leq i \leq 4$. Representatives for these four isomorphism classes are three groups $G_{0}^{4}(0,0), G_{0}^{4}(0,1), G_{0}^{4}(-1,0)$, whose abelian maximal normal subgroup is nearly homocyclic of type $(9,3)$, and the exceptional group $G_{0}^{4}(1,0) \simeq \operatorname{Syl}_{3} A_{9}$, which has the elementary abelian 3 -group of type $(3,3,3)$ as its abelian maximal normal subgroup. See the level of order $3^{4}=81$, i. e. the stem of $\Phi_{3}$, in Figure 1

For $G$ of coclass $\operatorname{cc}(G) \geq 2$, it remains to investigate the structure of the following 3-class groups:

- $\mathrm{Cl}_{3}\left(N_{1}\right)$ of order $3^{m-k-1}=3^{3}$ for small values of the index of nilpotency $m$, namely $m=4$ (where $\left[\chi_{s}(G), \gamma_{e}(G)\right]=1, k=0$ ), and $m=5$ if $\left[\chi_{s}(G), \gamma_{e}(G)\right]=\gamma_{m-1}(G), k=1$,

- $\mathrm{Cl}_{3}\left(N_{2}\right)$ of order $3^{e}=3^{3}$ for groups $G$ of coclass cc $(G)=2$ (where $e=3$ ),

- $\mathrm{Cl}_{3}\left(N_{i}\right), 3 \leq i \leq 4$, of order $3^{3}$ without restrictions for the parameters $m \geq 4$ and $e \geq 3$.

TABLE 1. 3-class groups to be investigated for certain parameters $m, n, e, k$ of $G$

\begin{tabular}{|c|c|c|c|cccc|}
\hline$m$ & $n$ & $e$ & $k$ & $\mathrm{Cl}_{3}\left(N_{1}\right)$ & $\mathrm{Cl}_{3}\left(N_{2}\right)$ & $\mathrm{Cl}_{3}\left(N_{3}\right)$ & $\mathrm{Cl}_{3}\left(N_{4}\right)$ \\
\hline 4 & 5 & 3 & 0 & $\times$ & $\times$ & $\times$ & $\times$ \\
5 & 6 & 3 & 1 & $\times$ & $\times$ & $\times$ & $\times$ \\
\hline 5 & 6 & 3 & 0 & & $\times$ & $\times$ & $\times$ \\
$\geq 6$ & $\geq 7$ & 3 & $\geq 0$ & & $\times$ & $\times$ & $\times$ \\
\hline 5 & 7 & 4 & 0 & & & $\times$ & $\times$ \\
$\geq 6$ & $\geq 8$ & $\geq 4$ & $\geq 0$ & & & $\times$ & $\times$ \\
\hline
\end{tabular}


In Table 1] we give an overview of all systems $(m, n, e, k)$ of parameters of the second 3-class group $G$ of coclass cc $(G) \geq 2$, for which the 3 -class groups $\mathrm{Cl}_{3}\left(N_{i}\right), 1 \leq i \leq 4$, marked by the symbol $\times$ have to be analyzed.

\section{Searching For 3-Class groups of 3-RAnK three}

Several authors, namely Scholz [39, p. 218], Kisilevsky [24, Thm. 3, p. 205], Heider and Schmithals [22, Satz 7, p. 11], and Brink [11, pp. 51-52], have pointed out the theoretical possibility that the elementary abelian 3-group of order 27 can occur as the 3-class group $\mathrm{Cl}_{3}(N)$ of an unramified cyclic cubic extension field $N$ of a base field $K$ with 3 -class group $\mathrm{Cl}_{3}(K)$ of type $(3,3)$. The most explicit result among these statements is due to Heider and Schmithals. They prove that the occurrence of an elementary abelian 3-class group $\mathrm{Cl}_{3}(N)$ of order 27 is restricted to extensions $N \mid K$ satisfying condition (B) in the sense of Taussky [42, p. 435], that is, having a partial principalization without fixed point [31, $\S 2.2$, p. 476].

Since the nearly homocyclic abelian 3-groups $\mathrm{A}(3, n)$ with $n \geq 2$, which generally occur as $\mathrm{Cl}_{3}(N)$, according to the theorems 3.1 and 3.2, are of 3-rank two, the elementary abelian 3-group of type $(3,3,3)$ is the unique possiblity for $\mathrm{Cl}_{3}(N)$ to be of 3-rank three. In contrast, many other possibilities arise when $\mathrm{Cl}_{3}(K)$ is of type $(9,3)[34$.

Unfortunately, it was impossible to find a numerical example, let alone a general criterion, for the occurrence of 3 -class groups of type $(3,3,3)$ in the bibliography, up to now. At first it was completely unknown, whether 3-class groups of type $(3,3,3)$ exist at all, if they appear sporadically or stochastically, or if their occurrence is ruled by deterministic laws.

In the present paper we systematically analyze this question by means of presentations for the second 3-class group $G=\operatorname{Gal}\left(\mathrm{F}_{3}^{2}(K) \mid K\right)$, which have been given by Blackburn [9, pp. 82-84] in the case of coclass $\operatorname{cc}(G)=1$ and by Nebelung [36, Satz 3.4.5, p. 94] (see our appendix) in the case of coclass $\operatorname{cc}(G) \geq 2$. We arrive at the surprising result that the transfer target type (TTT) $\tau(K)$, resp. the number $\varepsilon=\varepsilon(K)$ of elementary abelian 3-groups of type $(3,3,3)$ among the 3-class groups $\mathrm{Cl}_{3}\left(N_{i}\right), 1 \leq i \leq 4$, is connected with the transfer kernel type (TKT) $\varkappa(K)$, that is the principalization type of 3 -classes of $K$ in $N_{i}, 1 \leq i \leq 4$, by strict rules.

These connections offer new algorithmic possibilities for computing the principalization type of 3-classes of a quadratic base field $K=\mathbb{Q}(\sqrt{D})$ with 3-class group of type $(3,3)$, independently from the classical algorithm for complex quadratic fields by Scholz and Taussky [40] and its modification for real quadratic fields by Heider and Schmithals 22 . The new algorithm is based on determining the structure of the 3-class groups $\mathrm{Cl}_{3}(N)$ of the unramified cyclic cubic extensions $N \mid K$, that is, of four $S_{3}$-fields $N_{1}, \ldots, N_{4}$ of absolute degree six, and is described in $\S 5$.

With the aid of an implementation of this algorithm in the number theoretical computer algebra system PARI/GP 38, we have computed the principalization type of 3-classes of all 4596 quadratic base fields $K$ with 3 -class group of type $(3,3)$ and discriminant $-10^{6}<D<10^{7}$. The resulting extensive statistics of principalization types and second 3-class groups $G=\operatorname{Gal}\left(\mathrm{F}_{3}^{2}(K) \mid K\right)$ will be presented in $\S 6$

As in $\S$ 3, we distinguish second 3-class groups $G=\operatorname{Gal}_{3}\left(\mathrm{~F}_{3}^{2}(K) \mid K\right)$ of $\operatorname{coclass} \operatorname{cc}(G)=1$ in $\S 4.1$ and of coclass $\operatorname{cc}(G) \geq 2$ in $\S 4.2$.

\subsection{Second 3-class groups $G$ of coclass $\operatorname{cc}(G)=1$.}

Theorem 4.1. (Transfer target type $\tau(G)$ for groups $G$ with $\operatorname{cc}(G)=1, \operatorname{cl}(G) \leq 4$ )

The structure of the 3 -class groups $\mathrm{Cl}_{3}\left(N_{i}\right)$ of the four unramified cyclic cubic extensions $N_{i}$ $(1 \leq i \leq 4)$ of an arbitrary base field $K$ with 3 -class group $\mathrm{Cl}_{3}(K)$ of type $(3,3)$ and second 3 -class group $G=\operatorname{Gal}\left(\mathrm{F}_{3}^{2}(K) \mid K\right)$ of coclass $\operatorname{cc}(G)=1$ and order $|G|=3^{m}$ ist given by the following array for small indices of nilpotency $3 \leq m=\operatorname{cl}(G)+1 \leq 5$.

In the case $m=3$, it is supposed that the generating element $y$ of the maximal normal subgroup $M_{1}=\operatorname{Gal}\left(\mathrm{F}_{3}^{2}(K) \mid N_{1}\right)=\left\langle y, s_{2}\right\rangle$ of $G$ has order 3 . 


$$
\begin{aligned}
& \mathrm{Cl}_{3}\left(N_{1}\right) \simeq \begin{cases}\mathrm{A}(3,2), & \text { if } m=3, \\
\mathrm{~A}(3,3), & \text { if } m=4, G \nsucceq G_{0}^{4}(1,0) \simeq \mathrm{Syl}_{3} A_{9}, \\
C_{3} \times C_{3} \times C_{3}, & \text { if } m=4, G \simeq G_{0}^{4}(1,0) \simeq \mathrm{Syl}_{3} A_{9}, \\
\mathrm{~A}(3,3), & \text { if } k=1, m=5,\end{cases} \\
& \mathrm{Cl}_{3}\left(N_{i}\right) \simeq\left\{\begin{array}{ll}
\mathrm{A}(3,2), & \text { if } G \simeq G_{0}^{3}(0,0), \\
C_{9}, & \text { if } G \simeq G_{0}^{3}(0,1),
\end{array} \text { for } 2 \leq i \leq 4, \text { if } m=3 .\right.
\end{aligned}
$$

Proof. We make use of the well-known properties of the six isomorphism classes of metabelian 3 -groups $G_{a}^{m}(z, w)$ of coclass cc $(G)=1$ with the smallest indices of nilpotency $3 \leq m \leq 4$.

We start with the distinguished extension $N_{1}$.

First, let $\left[\chi_{2}(G), \gamma_{2}(G)\right]=1$, that is $k=0$. Then the group $\Gamma_{1}$ is isomorphic to $G$ and is of order $\left|\Gamma_{1}\right|=3^{m}$, according to equation (11) and [30, Cor. 3.1, p. 476].

For $m=4$, the abelian maximal normal subgroup $A_{1} \simeq M_{1}$ is either nearly homocyclic of type $(9,3)$ and thus isomorphic to $\mathrm{A}(3,3)$, if $G$ belongs to one of the three isomorphism classes $G_{0}^{4}(0,0), G_{0}^{4}(0,1), G_{0}^{4}(-1,0)$, or elementary abelian of type $(3,3,3)$, if $G$ is isomorphic to $G_{0}^{4}(1,0)$, the 3-Sylow subgroup $\mathrm{Syl}_{3} A_{9}$ of the alternating group of degree 9. See the stem of $\Phi_{3}$ in Figure 1

However, for $m=3$ the structure of the abelian maximal normal subgroup $A_{1} \simeq M_{1}$ can only be nearly homocyclic of type $(3,3)$ and thus isomorphic to $\mathrm{A}(3,2)$, in the case of the extra special group $G \simeq G_{0}^{3}(0,0)$ of exponent 3 with four abelian maximal normal subgroups of type $(3,3)$, as well as in the case of the extra special group $G \simeq G_{0}^{3}(0,1)$ of exponent 9 with one abelian maximal normal subgroup $A_{1} \simeq M_{1}=\left\langle y, s_{2}\right\rangle$ of type $(3,3)$, which is distinguished by our choice of the generating element $y$, and three further cyclic maximal normal subgroups of order 9 . See the level of order $3^{3}=27$, i. e. the stem of $\Phi_{2}$, in Figure 1

Now we consider $\left[\chi_{2}(G), \gamma_{2}(G)\right]=\gamma_{m-1}(G)$, that is $k=1$, with $m=5$ and thus $\gamma_{4}(G)>1$. According to equation (11) and [30. Cor. 3.1, p. 476], the group $\Gamma_{1} \simeq G / \gamma_{4}(G)$ is the immediate predecessor of $G$ [36, p. 182] on the coclass graph $\mathcal{G}(3,1)$. Thus it is isomorphic to $G_{0}^{4}(0,0)$ having the unique abelian maximal normal subgroup $A_{1} \simeq \mathrm{A}(3,3)$. See the vertex $\langle 81,9\rangle$ in Figure 1.

For the other three extensions $N_{i}$ with $2 \leq i \leq 4$, all groups $\Gamma_{i} \simeq G / \gamma_{3}(G) \simeq G$ [30, Cor. 3.1, p. 476] coincide with $G$, since $\gamma_{3}(G)=1$ is trivial for $m=3$. The structure of the abelian maximal normal subgroup $A_{i} \simeq M_{i}$ is either of type $(3,3)$ and thus isomorphic to $\mathrm{A}(3,2)$, if $G$ is isomorphic to $G_{0}^{3}(0,0)$, or cyclic of order 9 , if $G$ is isomorphic to $G_{0}^{3}(0,1)$, taking into account that $M_{i}=\left\langle x y^{i-2}, s_{2}\right\rangle=\left\langle x y^{i-2}\right\rangle$ with elements $x y^{i-2}$ of order 9 whose third power coincides with $s_{2}$. See the vertices $\langle 27,3\rangle$ and $\langle 27,4\rangle$ in Figure 1.

Corollary 4.1.1. (TTT $\tau(G)$ of $C_{3} \times C_{3}$ and stem groups $G$ in isoclinism families $\Phi_{2}, \Phi_{3}, \Phi_{10}$ ) Table Q gives the structure of 3 -class groups $\mathrm{Cl}_{3}\left(N_{i}\right), 1 \leq i \leq 4$, and invariant $\varepsilon=\#\{1 \leq i \leq 4 \mid$ $\left.\mathrm{Cl}_{3}\left(N_{i}\right) \simeq(3,3,3)\right\}$, for 3-groups $G \in \mathcal{G}(3,1)$ of small nilpotency class $1 \leq \mathrm{cl}(G)=m-1 \leq 4$ in dependence on the principalization or transfer kernel type (TKT) $\varkappa$ [31, Thm. 2.4-2.5, p. 478].

TABLE 2. TTT $\tau(G)$ for $G$ of coclass $\operatorname{cc}(G)=1$ and index of nilpotency $2 \leq m \leq 5$

\begin{tabular}{|cc|cc|c|cccc|c|}
\hline$m$ & $k$ & Type & $\varkappa$ & $\mathrm{Cl}_{3}\left(\mathrm{~F}_{3}^{1}(K)\right)$ & $\mathrm{Cl}_{3}\left(N_{1}\right)$ & $\mathrm{Cl}_{3}\left(N_{2}\right)$ & $\mathrm{Cl}_{3}\left(N_{3}\right)$ & $\mathrm{Cl}_{3}\left(N_{4}\right)$ & $\varepsilon$ \\
\hline 2 & 0 & $\mathrm{a} .1$ & $(0000)$ & 1 & $(3)$ & $(3)$ & $(3)$ & $(3)$ & 0 \\
\hline 3 & 0 & a.1 & $(0000)$ & $(3)$ & $(3,3)$ & $(3,3)$ & $(3,3)$ & $(3,3)$ & 0 \\
3 & 0 & A.1 & $(1111)$ & $(3)$ & $(3,3)$ & $(9)$ & $(9)$ & $(9)$ & 0 \\
\hline 4 & 0 & a.1 & $(0000)$ & $(3,3)$ & $(9,3)$ & $(3,3)$ & $(3,3)$ & $(3,3)$ & 0 \\
4 & 0 & a.2 & $(1000)$ & $(3,3)$ & $(9,3)$ & $(3,3)$ & $(3,3)$ & $(3,3)$ & 0 \\
4 & 0 & a.3 & $(2000)$ & $(3,3)$ & $(9,3)$ & $(3,3)$ & $(3,3)$ & $(3,3)$ & 0 \\
4 & 0 & a.3* & $(2000)$ & $(3,3)$ & $(3,3,3)$ & $(3,3)$ & $(3,3)$ & $(3,3)$ & 1 \\
\hline 5 & 1 & a.1 & $(0000)$ & $(9,3)$ & $(9,3)$ & $(3,3)$ & $(3,3)$ & $(3,3)$ & 0 \\
\hline
\end{tabular}


Example 4.1. The first occurrences of second 3-class groups $G=\operatorname{Gal}\left(\mathrm{F}_{3}^{2}(K) \mid K\right)$ of $\operatorname{coclass} \operatorname{cc}(G)=$ 1 with invariants $m=n=4, e=2$ for real quadratic fields $K=\mathbb{Q}(\sqrt{D})$ with discriminant $0<D<10^{7}$ and 3 -class group of type $(3,3)$ turned out to be the following. The smallest discriminant $D$ with TKT a.3, resp. a.2, is 32009 , resp. 72329 , according to [22, Tbl. 7, p. 24]. The smallest discriminant $D$ with TKT a.3*, is 142 097, known from [29, Part IV]. However, its special feature $\varepsilon=1$, resp. $\mathrm{Cl}_{3}\left(N_{1}\right) \simeq C_{3} \times C_{3} \times C_{3}$, was unknown up to 2009 .

Conjecture 4.1. For quadratic fields, the TKT a.1 cannot occur with a second 3-class group of defect $k=0, i$. e., an infinitely capable vertex located on the mainline of coclass graph $\mathcal{G}(3,1)$.

4.2. Second 3-class groups $G$ of coclass $\operatorname{cc}(G) \geq 2$. Suppose the order of a 3-class group $\mathrm{Cl}_{3}\left(N_{i}\right) \simeq M_{i} / \gamma_{2}\left(M_{i}\right)$ with $1 \leq i \leq 4$ has turned out to be 27 . The basic idea for deciding whether this 3 -class group is elementary abelian of type $(3,3,3)$ or nearly homocyclic of type $(9,3)$ consists in estimating the order of the cosets $\bar{v}=v \gamma_{2}\left(M_{i}\right)$ of the generators $v \in$ $\left\{g_{i}, s_{2}, \sigma_{3}, \ldots, \sigma_{m-1}, \tau_{3}, \ldots, \tau_{e}\right\}$ of $M_{i}=\left\langle g_{i}, \gamma_{2}(G)\right\rangle$ [30, Thm. 3.3, Proof, pp. 478-479] with respect to the commutator subgroup $\gamma_{2}\left(M_{i}\right)$. If all these orders are bounded from above by 3 , then we have an elementary abelian 3 -class group of type $(3,3,3)$, otherwise a nearly homocyclic 3 -class group of type $(9,3)$.

To reduce the investigation to the most important generator $g_{i}$, we first summarize general facts concerning the columns of Table 1 , that is, the four 3-class groups $\mathrm{Cl}_{3}\left(N_{i}\right), 1 \leq i \leq 4$, in the following three $\S \S 4.2 .14 .2 .3$

4.2.1. The distinguished 3-class group $\mathrm{Cl}_{3}\left(N_{1}\right) \simeq M_{1} / \gamma_{2}\left(M_{1}\right)$. According to 30, Cor. 3.2, p. 480], the maximal subgroup $M_{1}<G=\langle x, y\rangle$ with generator $y$, distinguished by the conditions $\gamma_{3}(G)=\left\langle y^{3}, x^{3}, \gamma_{4}(G)\right\rangle$ and $y \in \chi_{s}(G) \backslash \gamma_{2}(G)$, has the following properties.

$$
\begin{aligned}
M_{1} & =\left\langle y, \gamma_{2}(G)\right\rangle=\left\langle y, s_{2}, \sigma_{3}, \ldots, \sigma_{m-1}, \tau_{3}, \ldots, \tau_{e}\right\rangle, \\
\gamma_{2}\left(M_{1}\right) & =\left\langle t_{3}, \tau_{4}, \ldots, \tau_{e+1}\right\rangle .
\end{aligned}
$$

Since $\tau_{4}, \ldots, \tau_{e} \in \gamma_{2}\left(M_{1}\right)$ for $e \geq 4$, the order of the cosets $\overline{\tau_{4}}, \ldots, \overline{\tau_{e}}$ equals 1 .

The relation $\tau_{3}^{3} \tau_{4}^{3} \tau_{5}=1$ for third powers [30, Thm. 3.3, Proof, p. 478] implies $\tau_{3}^{3}=\tau_{4}^{-3} \tau_{5}^{-1} \in$ $\gamma_{2}\left(M_{1}\right)$ and $\operatorname{ord}\left(\overline{\tau_{3}}\right) \leq 3$, for any $e \geq 3$.

For the order of the cosets $\overline{s_{2}}, \overline{\sigma_{3}}, \ldots, \overline{\sigma_{m-1}}$, we cannot ensure the upper bound 3 , in general. However, in the two cases $m=4, n=5, \rho=0$ and $m=5, n=6, \rho= \pm 1$ to be investigated, according to Table 1, this estimate is possible. For $m=4, n=5, \rho=0$, we have the nilpotency relation $\sigma_{4}=\sigma_{m}=1, \sigma_{3}^{3}=\sigma_{4}^{-3} \sigma_{5}^{-1}=1$, and $s_{2}^{3}=\sigma_{4} \tau_{4}^{-1}=1$ by (7), since $e=3, \rho=0$, and thus $\tau_{4}=\tau_{e+1}=1$. For $m=5, n=6, \rho= \pm 1$, we have the nilpotency relation $\sigma_{5}=\sigma_{m}=1$, $\sigma_{4}^{3}=\sigma_{5}^{-3} \sigma_{6}^{-1}=1, \sigma_{3}^{3}=\sigma_{4}^{-3} \sigma_{5}^{-1}=1$, and $s_{2}^{3}=\sigma_{4} \sigma_{m-1}^{-\rho \beta} \tau_{4}^{-1}=\sigma_{4}^{1-\rho(\beta-1)}$ by (7), where $\sigma_{4}=$ $\sigma_{m-1}=\tau_{e+1}^{-\rho}=\tau_{4}^{-\rho} \in \gamma_{2}\left(M_{1}\right)$.

Consequently, we must only determine the order of the coset of the generator $y$ with third power $y^{3}=\sigma_{3}$.

4.2.2. The distinguished 3-class group $\mathrm{Cl}_{3}\left(N_{2}\right) \simeq M_{2} / \gamma_{2}\left(M_{2}\right)$. By [30, Cor. 3.2, p. 480], the maximal subgroup $M_{2}<G=\langle x, y\rangle$ with generator $x$, distinguished by the conditions $\gamma_{3}(G)=$ $\left\langle y^{3}, x^{3}, \gamma_{4}(G)\right\rangle$ and $x \in G \backslash \chi_{s}(G)$ if $s<m-1$, has the following properties.

$$
\begin{aligned}
M_{2} & =\left\langle x, \gamma_{2}(G)\right\rangle=\left\langle x, s_{2}, \sigma_{3}, \ldots, \sigma_{m-1}, \tau_{3}, \ldots, \tau_{e}\right\rangle, \\
\gamma_{2}\left(M_{2}\right) & =\left\langle s_{3}, \sigma_{4}, \ldots, \sigma_{m-1}\right\rangle .
\end{aligned}
$$

Since $\sigma_{4}, \ldots, \sigma_{m-1} \in \gamma_{2}\left(M_{2}\right)$ for $m \geq 5$, the order of the cosets $\overline{\sigma_{4}}, \ldots, \overline{\sigma_{m-1}}$ equals 1 . The relation $\sigma_{3}^{3} \sigma_{4}^{3} \sigma_{5}=1$ for third powers [30, Thm. 3.3, Proof, p. 478] implies $\sigma_{3}^{3}=\sigma_{4}^{-3} \sigma_{5}^{-1} \in$ $\gamma_{2}\left(M_{1}\right)$ and $\operatorname{ord}\left(\overline{\sigma_{3}}\right) \leq 3$, for any $m \geq 4$.

Since only the case $e=3$ is to be investigated, according to Table 1 , we have $\tau_{3}^{3}=\tau_{4}^{-3}=\tau_{e+1}^{-3}=$ $\sigma_{m-1}^{3 \rho} \in \gamma_{2}\left(M_{2}\right)$ by (7), because $\rho=0$ for $m=4$ and $\sigma_{m-1} \in \gamma_{2}\left(M_{2}\right)$ for $m \geq 5$. 
Finally, we have $s_{2}^{3}=\sigma_{4} \sigma_{m-1}^{-\rho \beta} \tau_{4}^{-1}=\sigma_{4} \sigma_{m-1}^{-\rho(\beta-1)} \in \gamma_{2}\left(M_{2}\right)$, since $\sigma_{4} \in \gamma_{2}\left(M_{2}\right)$ for $m \geq 4, \rho=0$ for $m=4$, and $\sigma_{m-1} \in \gamma_{2}\left(M_{2}\right)$ for $m \geq 5$, whence ord $\left(\overline{s_{2}}\right) \leq 3$.

Therefore, it only remains to investigate the order of the coset of the generator $x$ with third power $x^{3}=\tau_{3}$.

4.2.3. The other 3 -class groups $\mathrm{Cl}_{3}\left(N_{i}\right) \simeq M_{i} / \gamma_{2}\left(M_{i}\right), 3 \leq i \leq 4$. According to 30, Cor. 3.2, p. 480], the maximal subgroups $M_{i}<G=\langle x, y\rangle$ with $3 \leq i \leq 4$ with generators $x y$ and $x y^{-1}$, having third powers $(x y)^{3},\left(x y^{-1}\right)^{3} \in \zeta_{1+k}(G)$ in the first or second centre of $G$ [36, Lem. 3.4.11, p. 105], have the following properties.

$$
\begin{aligned}
M_{3} & =\left\langle x y, \gamma_{2}(G)\right\rangle=\left\langle x y, s_{2}, \sigma_{3}, \ldots, \sigma_{m-1}, \tau_{3}, \ldots, \tau_{e}\right\rangle, \\
\gamma_{2}\left(M_{3}\right) & =\left\langle s_{3} t_{3}, \gamma_{4}(G)\right\rangle \\
M_{4} & =\left\langle x y^{-1}, \gamma_{2}(G)\right\rangle=\left\langle x y^{-1}, s_{2}, \sigma_{3}, \ldots, \sigma_{m-1}, \tau_{3}, \ldots, \tau_{e}\right\rangle, \\
\gamma_{2}\left(M_{4}\right) & =\left\langle s_{3} t_{3}^{-1}, \gamma_{4}(G)\right\rangle, \\
\gamma_{4}(G) & =\left\langle\sigma_{4}, \ldots, \sigma_{m-1}, \tau_{4}, \ldots, \tau_{e}\right\rangle .
\end{aligned}
$$

Since $\sigma_{4}, \ldots, \sigma_{m-1}, \tau_{4}, \ldots, \tau_{e} \in \gamma_{4}(G)<\gamma_{2}\left(M_{i}\right)$ for $e \geq 4, m \geq 5$, the order of the cosets $\overline{\sigma_{4}}, \ldots, \overline{\sigma_{m-1}}$ and $\overline{\tau_{4}}, \ldots, \overline{\tau_{e}}$ equals 1 .

Due to the relations $\sigma_{3}^{3} \sigma_{4}^{3} \sigma_{5}=1$ and $\tau_{3}^{3} \tau_{4}^{3} \tau_{5}=1$ for third powers [30, Thm. 3.3, Proof, p. 478], we have ord $\left(\overline{\sigma_{3}}\right) \leq 3$ and $\operatorname{ord}\left(\overline{\tau_{3}}\right) \leq 3$.

Finally, we have $s_{2}^{3}=\sigma_{4} \sigma_{m-1}^{-\rho \beta} \tau_{4}^{-1} \in \gamma_{2}\left(M_{i}\right)$ by (7), since $\sigma_{4}, \tau_{4} \in \gamma_{2}\left(M_{i}\right)$ for $m \geq 4, e \geq 3, \rho=0$ for $m=4$ and $\sigma_{m-1} \in \gamma_{2}\left(M_{i}\right)$ for $m \geq 5$, whence ord $\left(\overline{s_{2}}\right) \leq 3$.

Thus, it only remains to determine the order of the coset of the generator $x y$, resp. $x y^{-1}$, for $i=3$, resp. $i=4$. We summarize the results of $\S \S 4.2 .14 .2 .3$ in Lemma 4.1.

Lemma 4.1. For each of the four 3-class groups $\mathrm{Cl}_{3}\left(N_{i}\right) \simeq M_{i} / \gamma_{2}\left(M_{i}\right), 1 \leq i \leq 4$, the decision whether $\mathrm{Cl}_{3}\left(N_{i}\right)$ is of type $(9,3)$ or of type $(3,3,3)$, in the case of 3 -class number $\mathrm{h}_{3}\left(N_{i}\right)=3^{3}$, exclusively depends on the order of the generator $g_{i}$ of $M_{i}=\left\langle g_{i}, \gamma_{2}(G)\right\rangle$ with respect to the commutator subgroup $\gamma_{2}\left(M_{i}\right)$.

The order of all the generators $s_{2}, \sigma_{3}, \ldots, \sigma_{m-1}, \tau_{3}, \ldots, \tau_{e}$ of $\gamma_{2}(G)$ with respect to $\gamma_{2}\left(M_{i}\right)$ is uniformly bounded from above by 3 .

After the preliminaries in the last three sections we come to the details of the rows of Table 1 in the following four sections.

To get an adequate view of $\S \S 4.34 .5$ it is useful to visualize that part of coclass graph $\mathcal{G}(3,2)$ which consists of 3 -groups $G$ of coclass $\operatorname{cc}(G)=2$ with abelianization $G / \gamma_{2}(G)$ of type $(3,3)$ and small order $|G|=3^{n}$ in Figure2. The groups $C_{3} \times C_{3}$ and $G_{0}^{3}(0,0)$ form the top of the mainline of $\mathcal{G}(3,1)$ in Figure 1. The edges of depth 2 neither belong to $\mathcal{G}(3,1)$ nor to $\mathcal{G}(3,2)$. The top of $\mathcal{G}(3,2)$ at the level of order $3^{5}=243$ consists of two isolated vertices $\langle 5\rangle,\langle 7\rangle$, two roots $\langle 9\rangle,\langle 4\rangle$ of finite trees, a root $\langle 3\rangle$ of an infinite tree, and two roots $\langle 6\rangle,\langle 8\rangle$ of coclass trees. Only the mainlines of infinite trees are shown. Vertices denoted by contour circles are metabelian [36, p. 189 ff.]. Groups with defect $k=0$ are represented by bigger circles than those with $k=1$. Vertices denoted by small contour squares are non-metabelian [4, Fig. 4.6-4.7, p. 74]. The symbol $n *$ denotes a batch of $n$ siblings below a common parent. Numbers in angles denote the identifiers of groups in the SmallGroup library [8] and in GAP 4.4 [20, where we omit the orders, which are given on the left hand scale. The symbols $\Phi_{s}$ denote isoclinism families [21, 13, 23]. The principalization or transfer kernel types, briefly TKT, 31, Tbl. 6-7, p. 492-493] in rectangles concern the vertices located vertically above.

4.3. Groups $G$ of coclass $\operatorname{cc}(G)=2$ with bicyclic centre and $m=4, n=5$. This section corresponds to the first row of Table 1. Here we must investigate the abelianizations of all four maximal subgroups $M_{i}, 1 \leq i \leq 4$. These 7 groups $\langle 243, i\rangle, 3 \leq i \leq 9$, form the stem of Hall's isoclinism family $\Phi_{6}$ [21, p. 139], 23, 4.1, p. 618, and 4.5 (6), pp. 620-621], 6, pp. 182-183] and satisfy the following special relations, by (77): 
FIGURE 2. Sporadic groups and roots of coclass trees on the coclass graph $\mathcal{G}(3,2)$

Order $3^{n}$

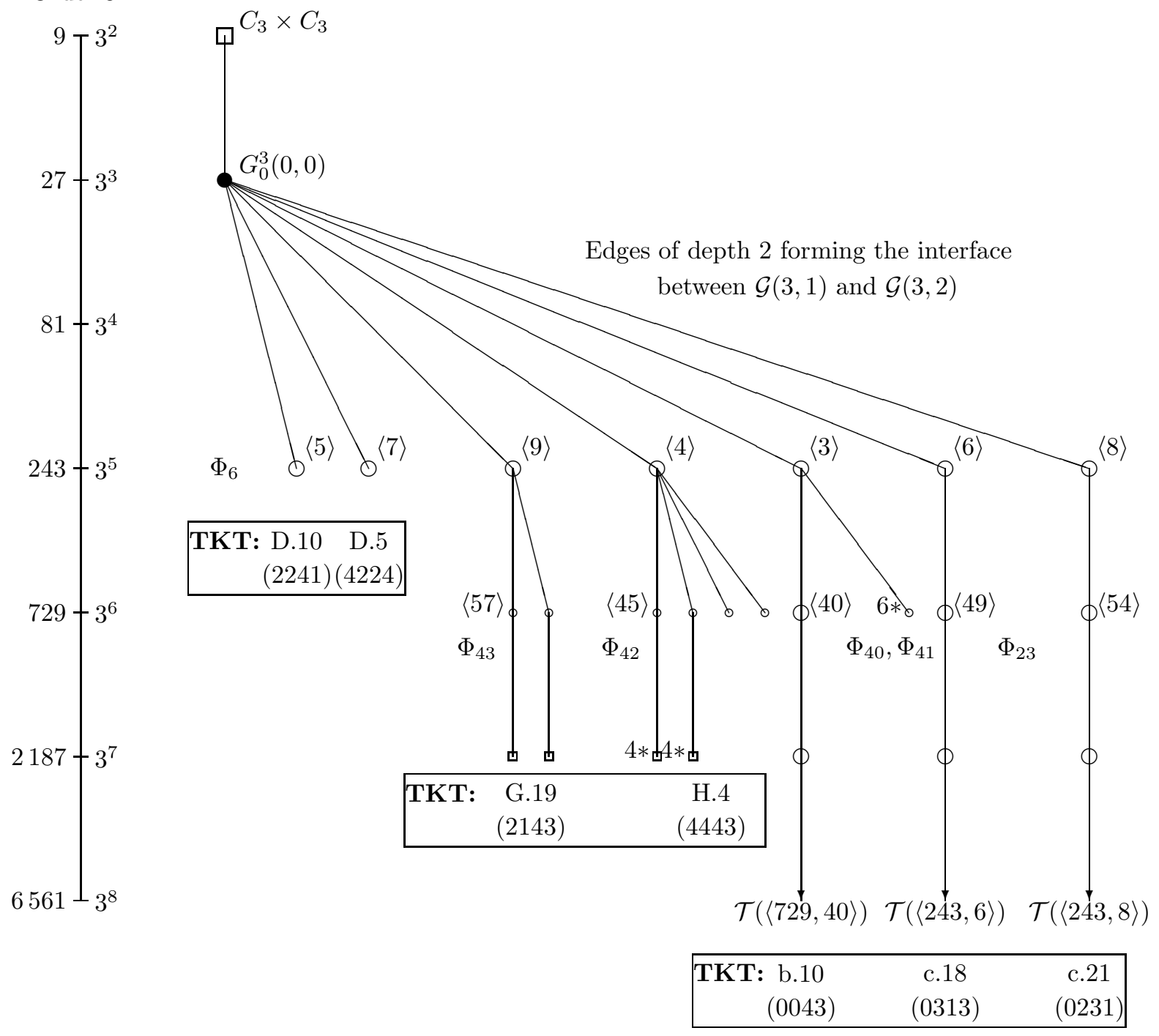

$$
\begin{aligned}
n & =5=2 m-3 \\
s=e & =n-m+2=3=m-1, \\
{\left[\chi_{s}(G), \gamma_{e}(G)\right] } & =\left[G, \gamma_{3}(G)\right]=\gamma_{4}(G)=1, k=0, \rho=0, \\
\sigma_{4} & =1, \tau_{4}=1 \\
\sigma_{3}^{3} & =1, \tau_{3}^{3}=1 \\
s_{2}^{3} & =1 \\
\gamma_{2}(G) & =\left\langle s_{2}\right\rangle \times\left\langle\sigma_{3}\right\rangle \times\left\langle\tau_{3}\right\rangle \text { of type }(3,3,3), \\
\gamma_{3}(G) & =\left\langle\sigma_{3}\right\rangle \times\left\langle\tau_{3}\right\rangle=\zeta_{1}(G) \text { of type }(3,3) .
\end{aligned}
$$

The commutator subgroups of the maximal normal subgroups are given by [30, Cor. 3.2, p. 480] and (7): 


$$
\begin{aligned}
& \gamma_{2}\left(M_{1}\right)=\left\langle t_{3}\right\rangle=\left\langle\sigma_{3}^{-\alpha} \tau_{3}^{1-\beta}\right\rangle, \\
& \gamma_{2}\left(M_{2}\right)=\left\langle s_{3}\right\rangle=\left\langle\sigma_{3}^{\gamma-1} \tau_{3}^{\delta}\right\rangle \\
& \gamma_{2}\left(M_{3}\right)=\left\langle s_{3} t_{3}\right\rangle=\left\langle\sigma_{3}^{\gamma-\alpha-1} \tau_{3}^{\delta-\beta+1}\right\rangle \\
& \gamma_{2}\left(M_{4}\right)=\left\langle s_{3} t_{3}^{-1}\right\rangle=\left\langle\sigma_{3}^{\alpha+\gamma-1} \tau_{3}^{\beta+\delta-1}\right\rangle .
\end{aligned}
$$

TABle 3. Parameters, third powers, and generators for $m=4, n=5, e=3, k=0$

\begin{tabular}{|l|crrr|cccc|cccc|}
\hline Type & $\alpha$ & $\beta$ & $\gamma$ & $\delta$ & $y^{3}$ & $x^{3}$ & $(x y)^{3}$ & $\left(x y^{-1}\right)^{3}$ & $t_{3}$ & $s_{3}$ & $s_{3} t_{3}$ & $s_{3} t_{3}^{-1}$ \\
\hline D.10 & 0 & 0 & -1 & 1 & $\sigma_{3}$ & $\tau_{3}$ & $\sigma_{3}^{-1} \tau_{3}$ & $\sigma_{3} \tau_{3}^{-1}$ & $\tau_{3}$ & $\sigma_{3}^{-2} \tau_{3}$ & $\sigma_{3}^{-2} \tau_{3}^{2}$ & $\sigma_{3}^{-2}$ \\
D.5 & 1 & 1 & -1 & 1 & $\sigma_{3}$ & $\tau_{3}$ & $\tau_{3}^{2}$ & $\sigma_{3}^{2}$ & $\sigma_{3}^{-1}$ & $\sigma_{3}^{-2} \tau_{3}$ & $\tau_{3}$ & $\sigma_{3}^{-1} \tau_{3}$ \\
G.19 & 0 & -1 & -1 & 0 & $\sigma_{3}$ & $\tau_{3}$ & $\sigma_{3}^{-1} \tau_{3}^{-1}$ & $\sigma_{3} \tau_{3}^{-1}$ & $\tau_{3}^{2}$ & $\sigma_{3}^{-2}$ & $\sigma_{3}^{-2} \tau_{3}^{2}$ & $\sigma_{3}^{-2} \tau_{3}^{-2}$ \\
H.4 & 1 & 1 & 1 & 1 & $\sigma_{3}$ & $\tau_{3}$ & $\sigma_{3}^{2} \tau_{3}^{2}$ & 1 & $\sigma_{3}^{-1}$ & $\tau_{3}$ & $\sigma_{3}^{-1} \tau_{3}$ & $\sigma_{3} \tau_{3}$ \\
\hline b.10 & 0 & 0 & 0 & 0 & $\sigma_{3}$ & $\tau_{3}$ & 1 & 1 & $\tau_{3}$ & $\sigma_{3}^{-1}$ & $\sigma_{3}^{-1} \tau_{3}$ & $\sigma_{3}^{-1} \tau_{3}^{-1}$ \\
c.18 & 0 & -1 & 0 & 1 & $\sigma_{3}$ & $\tau_{3}$ & 1 & $\tau_{3}^{-2}$ & $\tau_{3}^{2}$ & $\sigma_{3}^{-1} \tau_{3}$ & $\sigma_{3}^{-1}$ & $\sigma_{3}^{-1} \tau_{3}^{-1}$ \\
c. 21 & 0 & 0 & 0 & 1 & $\sigma_{3}$ & $\tau_{3}$ & $\tau_{3}$ & $\tau_{3}^{-1}$ & $\tau_{3}$ & $\sigma_{3}^{-1} \tau_{3}$ & $\sigma_{3}^{-1} \tau_{3}^{2}$ & $\sigma_{3}^{-1}$ \\
\hline
\end{tabular}

In Table 3 we calculate the third powers $g_{i}^{3}$ of the generators $g_{1}=y, g_{2}=x, g_{3}=x y, g_{4}=x y^{-1}$ of the maximal normal subgroups $M_{i}=\left\langle g_{i}, \gamma_{2}(G)\right\rangle$ and the generators of the commutator subgroups $\gamma_{2}\left(M_{i}\right)$ with $1 \leq i \leq 4$ for each of the 7 isomorphism classes of groups $G$ with $m=4, n=5$, and parameters $\alpha, \beta, \gamma, \delta$ given by [37, pp. 1-3]. The principalization types [36, Satz 6.14, p. 208], [31, Tbl. 6-7, pp. 492-493] of these isomorphism classes are all different.

Generally, according to [36, Lem. 3.4.11, p. 105], the third powers of $g_{3}$ and $g_{4}$ are given by $(x y)^{3}=\sigma_{3}^{\alpha+\gamma} \tau_{3}^{\beta+\delta}$ and $\left(x y^{-1}\right)^{3}=\sigma_{3}^{\alpha-\gamma} \tau_{3}^{\beta-\delta}$.

The order of the coset of $g_{i} \in M_{i}$ with respect to $\gamma_{2}\left(M_{i}\right)$ is bounded from above by 3 , if and only if the third power $g_{i}^{3}$ is contained in $\gamma_{2}\left(M_{i}\right)$.

Theorem 4.2. (Transfer target type $\tau(G)$ of stem groups $G$ in isoclinism family $\Phi_{6}$ )

Let $K$ be a number field with 3 -class group $\mathrm{Cl}_{3}(K)$ of type $(3,3)$. Suppose that the second 3 -class group $G=\operatorname{Gal}\left(\mathrm{F}_{3}^{2}(K) \mid K\right)$ of $K$ is of order $|G|=3^{n}$ and of class $\operatorname{cl}(G)=m-1$, where $m=4$ and $n=5$, i. e., that $G$ is one of the seven top vertices of coclass graph $\mathcal{G}(3,2)$ in Figure 2 , with invariant $e=3$ and bicyclic centre $\zeta_{1}(G)$.

Then the structure of the 3 -class groups of the first Hilbert 3 -class field $\mathrm{F}_{3}^{1}(K)$ of $K$ and of the four unramified cyclic cubic extensions $N_{1}, \ldots, N_{4}$ of $K$ is given by Table 4 , in dependence on the principalization type $\varkappa$ of $K$. The invariant $\varepsilon=\varepsilon(K)$ denotes the number of elementary abelian 3 -class groups $\mathrm{Cl}_{3}\left(N_{i}\right)$ of type $(3,3,3)$, for each principalization type.

TABLE 4. 3 -class groups of type $(3,3,3)$ for $m=4, n=5, e=3, k=0$

\begin{tabular}{|lc|c|cccc|c|}
\hline Type & $\varkappa$ & $\mathrm{Cl}_{3}\left(\mathrm{~F}_{3}^{1}(K)\right)$ & $\mathrm{Cl}_{3}\left(N_{1}\right)$ & $\mathrm{Cl}_{3}\left(N_{2}\right)$ & $\mathrm{Cl}_{3}\left(N_{3}\right)$ & $\mathrm{Cl}_{3}\left(N_{4}\right)$ & $\varepsilon$ \\
\hline D.10 & $(2241)$ & $(3,3,3)$ & $(9,3)$ & $(9,3)$ & $(3,3,3)$ & $(9,3)$ & 1 \\
D.5 & $(4224)$ & $(3,3,3)$ & $(3,3,3)$ & $(9,3)$ & $(3,3,3)$ & $(9,3)$ & 2 \\
G.19 & $(2143)$ & $(3,3,3)$ & $(9,3)$ & $(9,3)$ & $(9,3)$ & $(9,3)$ & 0 \\
H.4 & $(4443)$ & $(3,3,3)$ & $(3,3,3)$ & $(3,3,3)$ & $(9,3)$ & $(3,3,3)$ & 3 \\
\hline b.10 & $(0043)$ & $(3,3,3)$ & $(9,3)$ & $(9,3)$ & $(3,3,3)$ & $(3,3,3)$ & 2 \\
c.18 & $(0313)$ & $(3,3,3)$ & $(9,3)$ & $(9,3)$ & $(3,3,3)$ & $(9,3)$ & 1 \\
c.21 & $(0231)$ & $(3,3,3)$ & $(9,3)$ & $(9,3)$ & $(9,3)$ & $(9,3)$ & 0 \\
\hline
\end{tabular}


Proof. The structure of the 3-class group $\mathrm{Cl}_{3}\left(\mathrm{~F}_{3}^{1}(K)\right)$ of the first Hilbert 3-class field of $K$ can be obtained from the parameters $m=4$ and $e=3$ by means of the following two isomorphisms from $\mathrm{Cl}_{3}\left(\mathrm{~F}_{3}^{1}(K)\right)$ to the commutator subgroup $\gamma_{2}(G)$ of $G=\operatorname{Gal}\left(\mathrm{F}_{3}^{2}(K) \mid K\right)$, according to [1] and [36. Satz 4.2 .4 , p. 131]

$$
\mathrm{Cl}_{3}\left(\mathrm{~F}_{3}^{1}(K)\right) \simeq \operatorname{Gal}\left(\mathrm{F}_{3}^{2}(K) \mid \mathrm{F}_{3}^{1}(K)\right) \simeq \gamma_{2}(G) \simeq \mathrm{A}(3, m-2) \times \mathrm{A}(3, e-2)=\mathrm{A}(3,2) \times \mathrm{A}(3,1)
$$

The structure of the 3 -class groups $\mathrm{Cl}_{3}\left(N_{i}\right)$ is a consequence of Table 3 , since we have the following isomorphism, according to [1]

$$
\mathrm{Cl}_{3}\left(N_{i}\right) \simeq \operatorname{Gal}\left(\mathrm{F}_{3}^{1}\left(N_{i}\right) \mid N_{i}\right) \simeq \operatorname{Gal}\left(\mathrm{F}_{3}^{2}(K) \mid N_{i}\right) / \operatorname{Gal}\left(\mathrm{F}_{3}^{2}(K) \mid \mathrm{F}_{3}^{1}\left(N_{i}\right)\right) \simeq M_{i} / \gamma_{2}\left(M_{i}\right) .
$$

Taking into consideration the preliminaries in $\S \S 4.2 .14 .2 .3$, resp. in Lemma 4.1, we use the equivalence of the following statements.

$$
\begin{aligned}
& \mathrm{Cl}_{3}\left(N_{1}\right) \simeq \mathrm{A}(3,3) \quad \Longleftrightarrow \quad g_{1}^{3}=y^{3} \notin\left\langle t_{3}\right\rangle=\gamma_{2}\left(M_{1}\right), \\
& \mathrm{Cl}_{3}\left(N_{2}\right) \simeq \mathrm{A}(3,3) \quad \Longleftrightarrow \quad g_{2}^{3}=x^{3} \notin\left\langle s_{3}\right\rangle=\gamma_{2}\left(M_{2}\right), \\
& \mathrm{Cl}_{3}\left(N_{3}\right) \simeq \mathrm{A}(3,3) \quad \Longleftrightarrow \quad g_{3}^{3}=(x y)^{3} \notin\left\langle s_{3} t_{3}\right\rangle=\gamma_{2}\left(M_{3}\right), \\
& \mathrm{Cl}_{3}\left(N_{4}\right) \simeq \mathrm{A}(3,3) \quad \Longleftrightarrow \quad g_{4}^{3}=\left(x y^{-1}\right)^{3} \notin\left\langle s_{3} t_{3}^{-1}\right\rangle=\gamma_{2}\left(M_{4}\right)
\end{aligned}
$$

Corollary 4.2.1. For each of these seven isomorphism classes of the second 3-class group G, a 3-class group $\mathrm{Cl}_{3}\left(N_{i}\right), 1 \leq i \leq 4$, is of type $(3,3,3)$ if and only if the norm class group $\operatorname{Norm}_{N_{i} \mid K}\left(\mathrm{Cl}_{3}\left(N_{i}\right)\right)$ becomes principal either in none or in three of the extensions $N_{\ell}, 1 \leq \ell \leq 4$. The extensions with 3 -class group of type $(3,3,3)$ always satisfy the condition (B) of Taussky 42 , i. e., they have a partial principalization without fixed point, as predicted by [22, Satz 7, p. 11].

Proof. This is an immediate consequence of the principalization types $\varkappa$ of these seven isomorphism classes. Let $\mathrm{j}_{N_{\ell} \mid K}: \mathrm{Cl}_{p}(K) \longrightarrow \mathrm{Cl}_{p}\left(N_{\ell}\right), 1 \leq \ell \leq 4$, denote the class extension homomorphisms [31, $\S 2.3$, p. 477]. We demonstrate two cases.

For type H.4, $\varkappa=(4443)$, we have $\operatorname{Norm}_{N_{i} \mid K}\left(\mathrm{Cl}_{3}\left(N_{i}\right)\right) \cap \operatorname{ker}\left(\mathrm{j}_{N_{\ell} \mid K}\right)=1$, for $i=1,2$ and any $1 \leq \ell \leq 4$, $\operatorname{Norm}_{N_{3} \mid K}\left(\mathrm{Cl}_{3}\left(N_{3}\right)\right)=\operatorname{ker}\left(\mathrm{j}_{N_{4} \mid K}\right)$, and $\operatorname{Norm}_{N_{4} \mid K}\left(\mathrm{Cl}_{3}\left(N_{4}\right)\right)=\operatorname{ker}\left(\mathrm{j}_{N_{\ell} \mid K}\right)$, for $1 \leq \ell \leq 3$.

For type b.10, $\varkappa=(0043)$, we have $\operatorname{Norm}_{N_{i} \mid K}\left(\mathrm{Cl}_{3}\left(N_{i}\right)\right)<\operatorname{ker}\left(\mathrm{j}_{N_{\ell} \mid K}\right)=\mathrm{Cl}_{3}(K)$, for any $1 \leq i \leq 4$ and $\ell=1,2$, and additionally $\operatorname{Norm}_{N_{3} \mid K}\left(\mathrm{Cl}_{3}\left(N_{3}\right)\right)=\operatorname{ker}\left(\mathrm{j}_{N_{4} \mid K}\right)$ and $\operatorname{Norm}_{N_{4} \mid K}\left(\mathrm{Cl}_{3}\left(N_{4}\right)\right)=\operatorname{ker}\left(\mathrm{j}_{N_{3} \mid K}\right)$.

Corollary 4.2.2. If $K$ is a quadratic base field with $G \in \Phi_{6}$, then the three total principalization types b.10, c.21, c.18 are impossible, due to class number relations, and the remaining four partial principalization types D.10, G.19, H.4, D.5 are characterized uniquely by the invariant $\varepsilon$.

Proof. For principalization types b.10, c.21, c.18 with singulet $\varkappa(1)=0[31, \S 2.2-2.3$, p. 475478], the entire 3-class group $\mathrm{Cl}_{3}(K)$ becomes principal in $N_{1}$. Hence, a quadratic base field $K$ must be real, and the unramified cyclic cubic extension $N_{1}$ must be an $S_{3}$-field of type $\alpha$ with even 3-exponent of the 3-class number $\mathrm{h}_{3}\left(N_{1}\right)$, in contradiction to $\mathrm{Cl}_{3}\left(N_{1}\right) \simeq \mathrm{A}(3,3)$ [30, Prop. $4.3-4.4$, p. 484-485].

Example 4.2. The first occurrences of second 3-class groups $G=\operatorname{Gal}\left(\mathrm{F}_{3}^{2}(K) \mid K\right)$ of $\operatorname{coclass} \operatorname{cc}(G)=$ 2 with invariants $m=4, n=5, e=3$ and bicyclic centre among quadratic fields $K=\mathbb{Q}(\sqrt{D})$ with discriminant $-10^{6}<D<0$, resp. $0<D<10^{7}$, and 3-class group of type $(3,3)$ turned out to be the following.

- The smallest value $|D|$ of the discriminant of a complex quadratic field $K$ with principalization type D.10, resp. D.5, is 4027 [40, pp. 22-25], resp. 12131 [22, Tbl. 3, p. $19]$.

- The smallest discriminant $D$ of a real quadratic field $K$ with principalization type D.10, resp. D.5, is 422573 , resp. 631769 . Both were unknown until 2006, resp. 2009. 
Conjecture 4.2. For quadratic base fields, the principalization types G.19 and H.4 cannot occur with invariants $m=4, n=5$, and $k=0$ of the corresponding second 3 -class groups $\langle 243,9\rangle$, $\langle 243,4\rangle$, since they have terminal metabelian descendants (Figure Q 2 ) of the same principalization type with invariants $m=5, n=6$, and bigger defect $k=1$. We call this the weak or restricted leaf conjecture. (See Thm. 1.4 and Cnj. 3.1 in [33.)

4.4. Groups $G$ of coclass $\operatorname{cc}(G)=2$ with cyclic centre and $m=5, n=6$. This section corresponds to the second row of Table 1 . Again, the abelianizations of all maximal subgroups $M_{i}, 1 \leq i \leq 4$, have to be analyzed. These groups form the stem of Easterfield's isoclinism families $\Phi_{40}, \Phi_{41}, \Phi_{42}, \Phi_{43}[13,[23,4.1$, p. 619 , and 4.6 (40)-(43), p. 636] and satisfy the following special relations, by (7):

$$
\begin{aligned}
n & =6=2 m-4, \\
s & =4=m-1, \\
e & =n-m+2=3=m-2, \\
{\left[\chi_{s}(G), \gamma_{e}(G)\right] } & =\left[G, \gamma_{3}(G)\right]=\gamma_{4}(G)=\gamma_{m-1}(G)>1, k=1, \rho= \pm 1, \\
\sigma_{5} & =1, \tau_{5}=1, \\
\sigma_{4}^{3} & =1, \tau_{4}^{3}=1, \\
\sigma_{3}^{3} & =\sigma_{4}^{-3}=1, \tau_{3}^{3}=\tau_{4}^{-3}=1, \\
s_{2}^{3} & =\sigma_{4}^{1-\rho(\beta-1)}, \\
s_{2}^{3} & =1 \Longleftrightarrow \beta=0, \rho=-1 \text { or } \beta=-1, \rho=1, \\
\tau_{4} & =\sigma_{4}^{-\rho},
\end{aligned}
$$

$$
\begin{aligned}
& \gamma_{2}(G)=\left\langle s_{2}, \sigma_{3}, \sigma_{4}, \tau_{3}\right\rangle \text { of type } \begin{cases}(9,3,3), & \text { if } s_{2}^{3} \neq 1, \\
(3,3,3,3), & \text { if } s_{2}^{3}=1,\end{cases} \\
& \gamma_{3}(G)=\left\langle\sigma_{3}, \sigma_{4}, \tau_{3}\right\rangle \text { of type }(3,3,3), \\
& \gamma_{4}(G)=\left\langle\sigma_{4}\right\rangle=\zeta_{1}(G) \text { of type }(3) .
\end{aligned}
$$

The commutator subgroups of the maximal normal subgroups are given by [30, Cor. 3.2, p. 480] and (7):

$$
\begin{aligned}
& \gamma_{2}\left(M_{1}\right)=\left\langle t_{3}, \sigma_{4}\right\rangle=\left\langle\sigma_{3}^{-\rho \delta} \sigma_{4}^{\rho-\alpha} \tau_{3}^{1-\beta}, \sigma_{4}\right\rangle=\left\langle\sigma_{3}^{-\rho \delta} \tau_{3}^{1-\beta}, \sigma_{4}\right\rangle, \\
& \gamma_{2}\left(M_{2}\right)=\left\langle s_{3}, \sigma_{4}\right\rangle=\left\langle\sigma_{3}^{\rho \beta-1} \sigma_{4}^{\gamma-1} \tau_{3}^{\delta}, \sigma_{4}\right\rangle=\left\langle\sigma_{3}^{\rho \beta-1} \tau_{3}^{\delta}, \sigma_{4}\right\rangle, \\
& \gamma_{2}\left(M_{3}\right)=\left\langle s_{3} t_{3}, \sigma_{4}\right\rangle=\left\langle\sigma_{3}^{\rho(\beta-\delta)-1} \tau_{3}^{\delta-\beta+1}, \sigma_{4}\right\rangle, \\
& \gamma_{2}\left(M_{4}\right)=\left\langle s_{3} t_{3}^{-1}, \sigma_{4}\right\rangle=\left\langle\sigma_{3}^{\rho(\beta+\delta)-1} \tau_{3}^{\beta+\delta-1}, \sigma_{4}\right\rangle .
\end{aligned}
$$

The dependencies on the parameters $\alpha, \gamma$ disappear, since they occur in the exponent of $\sigma_{4} \in$ $\gamma_{4}(G)$, but each $\gamma_{2}\left(M_{i}\right)$ with $1 \leq i \leq 4$ contains $\gamma_{4}(G)=\left\langle\sigma_{4}\right\rangle=\left\langle\tau_{4}\right\rangle$.

In Table 5 we calculate the third powers $g_{i}^{3}$ of the generators $g_{1}=y, g_{2}=x, g_{3}=x y, g_{4}=x y^{-1}$ of the maximal normal subgroups $M_{i}=\left\langle g_{i}, \gamma_{2}(G)\right\rangle$ and the generators of the commutator subgroups $\gamma_{2}\left(M_{i}\right), 1 \leq i \leq 4$, modulo $\sigma_{4}$ for each of the 12 isomorphism classes of groups $G$ with $m=5$, $n=6, \rho= \pm 1$ [37, pp. 4-7]. Several of these isomorphism classes have the same principalization type and the same parameters $\beta, \delta, \rho$, as indicated by the second column of Table 5 .

Generally, according to [36, Lem. 3.4.11, p. 105], the third powers of $g_{3}$ and $g_{4}$ modulo $\sigma_{4}$ are given by

$(x y)^{3}=\sigma_{3}^{\rho(\beta+\delta)} \sigma_{4}^{\alpha+\gamma+\rho(\beta+\delta)} \tau_{3}^{\beta+\delta} \equiv \sigma_{3}^{\rho(\beta+\delta)} \tau_{3}^{\beta+\delta}$ and $\left(x y^{-1}\right)^{3}=\sigma_{3}^{\rho(\delta-\beta)} \sigma_{4}^{\alpha-\gamma+\rho \beta} \tau_{3}^{\beta-\delta} \equiv \sigma_{3}^{\rho(\delta-\beta)} \tau_{3}^{\beta-\delta}$.

The order of the coset of $g_{i} \in M_{i}$ with respect to $\gamma_{2}\left(M_{i}\right)$ is bounded from above by 3 , if and only if the third power $g_{i}^{3}$ is contained in $\gamma_{2}\left(M_{i}\right)$. 
TABle 5. Parameters, third powers, and generators for $m=5, n=6, e=3, k=1$

\begin{tabular}{|l|c|rrr|cccc|cccc|}
\hline Type & isom. cl. & $\beta$ & $\delta$ & $\rho$ & $y^{3}$ & $x^{3}$ & $\overline{(x y)^{3}}$ & $\overline{\left(x y^{-1}\right)^{3}}$ & $\overline{t_{3}}$ & $\overline{s_{3}}$ & $\overline{s_{3} t_{3}}$ & $\overline{s_{3} t_{3}^{-1}}$ \\
\hline G.19 & 2 & -1 & 0 & 1 & $\sigma_{3}$ & $\tau_{3}$ & $\sigma_{3}^{-1} \tau_{3}^{-1}$ & $\sigma_{3} \tau_{3}^{-1}$ & $\tau_{3}^{2}$ & $\sigma_{3}^{-2}$ & $\sigma_{3}^{-2} \tau_{3}^{2}$ & $\sigma_{3}^{-2} \tau_{3}^{-2}$ \\
H.4 & 4 & 1 & 1 & 1 & $\sigma_{3}$ & $\tau_{3}$ & $\sigma_{3}^{2} \tau_{3}^{2}$ & 1 & $\sigma_{3}^{-1}$ & $\tau_{3}$ & $\sigma_{3}^{-1} \tau_{3}$ & $\sigma_{3} \tau_{3}$ \\
\hline b.10 & 3 & 0 & 0 & -1 & $\sigma_{3}$ & $\tau_{3}$ & 1 & 1 & $\tau_{3}$ & $\sigma_{3}^{-1}$ & $\sigma_{3}^{-1} \tau_{3}$ & $\sigma_{3}^{-1} \tau_{3}^{-1}$ \\
b.10 & 3 & 0 & 0 & 1 & $\sigma_{3}$ & $\tau_{3}$ & 1 & 1 & $\tau_{3}$ & $\sigma_{3}^{-1}$ & $\sigma_{3}^{-1} \tau_{3}$ & $\sigma_{3}^{-1} \tau_{3}^{-1}$ \\
\hline
\end{tabular}

Theorem 4.3. (TTT $\tau(G)$ of stem groups $G$ in isoclinism families $\left.\Phi_{40}, \Phi_{41}, \Phi_{42}, \Phi_{43}\right)$

Let $K$ be a number field with 3 -class group $\mathrm{Cl}_{3}(K)$ of type $(3,3)$. Suppose that the second 3 -class group $G=\operatorname{Gal}\left(\mathrm{F}_{3}^{2}(K) \mid K\right)$ is of order $|G|=3^{n}$ and class $\mathrm{cl}(G)=m-1$, where $n=6$ and $m=5$, such that $\left[\chi_{s}(G), \gamma_{e}(G)\right]=\gamma_{m-1}(G), k=1$, i.e., that $G$ is one of the twelve vertices with defect $k=1$ of coclass graph $\mathcal{G}(3,2)$ in Figure 2 , with invariant $e=3$ and cyclic centre $\zeta_{1}(G)$.

Then the structure of the 3-class groups of the first Hilbert 3 -class field $\mathrm{F}_{3}^{1}(K)$ of $K$ and of the four unramified cyclic cubic extensions $N_{1}, \ldots, N_{4}$ of $K$ is given by Table [6, in dependence on the principalization type $\varkappa$ of $K$ and on the relational parameters $\beta, \rho$ of $G$. The invariant $\varepsilon$ denotes the number of 3-class groups $\mathrm{Cl}_{3}\left(N_{i}\right)$ of type $(3,3,3)$, for each principalization type.

TABLE 6. 3-class groups of type $(3,3,3)$ for $m=5, n=6, e=3, k=1$

\begin{tabular}{|lc|rr|c|cccc|c|}
\hline Type & $\varkappa$ & $\beta$ & $\rho$ & $\mathrm{Cl}_{3}\left(\mathrm{~F}_{3}^{1}(K)\right)$ & $\mathrm{Cl}_{3}\left(N_{1}\right)$ & $\mathrm{Cl}_{3}\left(N_{2}\right)$ & $\mathrm{Cl}_{3}\left(N_{3}\right)$ & $\mathrm{Cl}_{3}\left(N_{4}\right)$ & $\varepsilon$ \\
\hline G.19 & $(2143)$ & -1 & 1 & $(3,3,3,3)$ & $(9,3)$ & $(9,3)$ & $(9,3)$ & $(9,3)$ & 0 \\
H.4 & $(4443)$ & 1 & 1 & $(9,3,3)$ & $(3,3,3)$ & $(3,3,3)$ & $(9,3)$ & $(3,3,3)$ & 3 \\
\hline b.10 & $(0043)$ & 0 & -1 & $(3,3,3,3)$ & $(9,3)$ & $(9,3)$ & $(3,3,3)$ & $(3,3,3)$ & 2 \\
b.10 & $(0043)$ & 0 & 1 & $(9,3,3)$ & $(9,3)$ & $(9,3)$ & $(3,3,3)$ & $(3,3,3)$ & 2 \\
\hline
\end{tabular}

Proof. Similarly as in the proof of Theorem 4.2, the structure of the 3-class group $\mathrm{Cl}_{3}\left(\mathrm{~F}_{3}^{1}(K)\right)$ of the first Hilbert 3-class field of $K$ is a consequence of $m=5, e=3$, and the isomorphisms in [1] and [36, Satz 4.2.4, p. 131]

$\mathrm{Cl}_{3}\left(\mathrm{~F}_{3}^{1}(K)\right) \simeq \gamma_{2}(G) \simeq\left\{\begin{array}{l}\mathrm{A}(3, m-2) \times \mathrm{A}(3, e-2)=\mathrm{A}(3,3) \times \mathrm{A}(3,1) \text { for } \rho \not \equiv \beta-1 \quad(\bmod 3), \\ \mathrm{A}(3, m-3) \times \mathrm{A}(3, e-1)=\mathrm{A}(3,2) \times \mathrm{A}(3,2) \text { for } \rho \equiv \beta-1 \quad(\bmod 3) .\end{array}\right.$

The structure of the 3 -class groups $\mathrm{Cl}_{3}\left(N_{i}\right) \simeq M_{i} / \gamma_{2}\left(M_{i}\right)$ follows from Table 5 , if we take into consideration the preparations in $\S \S 4.2 .14 .2 .3$, resp. in Lemma 4.1 .

Corollary 4.3.1. For each of these twelve isomorphism classes of the second 3-class group $G$, a 3-class group $\mathrm{Cl}_{3}\left(N_{i}\right), 1 \leq i \leq 4$, is of type $(3,3,3)$ if and only if the norm class group Norm $_{N_{i} \mid K}\left(\mathrm{Cl}_{3}\left(N_{i}\right)\right)$ becomes principal either in none or in three of the extensions $N_{\ell}, 1 \leq \ell \leq 4$. The extensions with 3 -class group of type $(3,3,3)$ always satisfy the condition (B) of Taussky [42], $i$. e., they have a partial principalization without fixed point.

Proof. This is an immediate consequence of the principalization types $\varkappa$ of these twelve isomorphism classes.

Corollary 4.3.2. If $K$ is a quadratic base field with $G \in \Phi_{s}$, for some $s \in\{40,41,42,43\}$, then the total principalization type b.10 is impossible, due to class number relations, and the remaining two partial principalization types G.19 and H.4 are characterised uniquely by the invariant $\varepsilon$. Furthermore, only the second 3-class groups $G=\langle 729,57\rangle$ and $G=\langle 729,45\rangle$ are possible.

Proof. For the principalization type b.10 with singulets $\varkappa(1)=\varkappa(2)=0$, the entire 3 -class group $\mathrm{Cl}_{3}(K)$ becomes principal in $N_{1}, N_{2}$. In the case of a quadratic base field $K$ this yields a similar contradiction to $\mathrm{Cl}_{3}\left(N_{1}\right) \simeq \mathrm{Cl}_{3}\left(N_{2}\right) \simeq \mathrm{A}(3,3)$ as in the proof of Corollary 4.2.2. For the last assertion, we refer to Thm. 3.14 in 33 . 
Example 4.3. The first occurrences of second 3-class groups $G=\operatorname{Gal}\left(\mathrm{F}_{3}^{2}(K) \mid K\right)$ of $\operatorname{coclass} \operatorname{cc}(G)=$ 2 with invariants $m=5, n=6, e=3, \rho= \pm 1$ and cyclic centre among quadratic fields $K=$ $\mathbb{Q}(\sqrt{D})$ with discriminant $-10^{6}<D<0$, resp. $0<D<10^{7}$, and 3 -class group of type $(3,3)$ turned out to be the following.

- The smallest value $|D|$ of the discriminant of a complex quadratic field $K$ with principalization type H.4, resp. G.19, is 3896, resp. 12067 [22, Tbl. 3, p. 19].

- The smallest discriminant $D$ of a real quadratic field $K$ with principalization type G.19, resp. H.4, is 214712, resp. 957 013. Both were unknown until 2006, resp. 2009.

4.5. All other groups $G$ of coclass $\operatorname{cc}(G)=2$ with $m \geq 5, n \geq 6$. This section corresponds to the third and fourth row of Table 1. Here we have to analyze the abelianizations of three maximal subgroups $M_{i}, 2 \leq i \leq 4$. These groups satisfy the following general relations. For $m \geq 6$, we have $\sigma_{m-2}, \sigma_{m-1} \in \gamma_{4}(G)$. For $m=5$, the case $\rho= \pm 1$ has been investigated in the preceding section already, and we only have to consider the remaining possibility $\rho=0$. Since $\sigma_{m-1} \in \gamma_{4}(G)$, also for $m=5$, the following congruences modulo $\gamma_{4}(G)$ are valid, generally. The apparent dependencies on parameters $\alpha, \gamma, \rho$ vanish. Here we use [36, Lem. 3.4.11, p. 105] and (7).

$$
\begin{aligned}
(x y)^{3} & =\sigma_{m-2}^{\rho(\beta+\delta)} \sigma_{m-1}^{\alpha+\gamma+\rho(\beta+\delta)} \tau_{3}^{\beta+\delta} \equiv \tau_{3}^{\beta+\delta} \quad\left(\bmod \gamma_{4}(G)\right) \\
\left(x y^{-1}\right)^{3} & =\sigma_{m-2}^{\rho(\delta-\beta)} \sigma_{m-1}^{\alpha-\gamma+\rho \beta} \tau_{3}^{\beta-\delta} \equiv \tau_{3}^{\beta-\delta} \quad\left(\bmod \gamma_{4}(G)\right) \\
t_{3} & =\tau_{3} \tau_{4} \tau_{3}^{-\beta} \sigma_{m-2}^{-\rho \delta} \sigma_{m-1}^{-\alpha} \equiv \tau_{3}^{1-\beta} \quad\left(\bmod \gamma_{4}(G)\right) \\
s_{3} & =\sigma_{3}^{-1} \sigma_{4}^{-1} \sigma_{m-2}^{\rho \beta} \sigma_{m-1}^{\gamma} \tau_{3}^{\delta} \equiv \sigma_{3}^{-1} \tau_{3}^{\delta} \quad\left(\bmod \gamma_{4}(G)\right) \\
s_{3} t_{3} & \equiv \sigma_{3}^{-1} \tau_{3}^{\delta-\beta+1} \quad\left(\bmod \gamma_{4}(G)\right) \\
s_{3} t_{3}^{-1} & \equiv \sigma_{3}^{-1} \tau_{3}^{\beta+\delta-1} \quad\left(\bmod \gamma_{4}(G)\right) .
\end{aligned}
$$

In Table 7 we calculate the third powers $g_{i}^{3}$ of the generators $g_{1}=y, g_{2}=x, g_{3}=x y, g_{4}=x y^{-1}$ of the maximal normal subgroups $M_{i}=\left\langle g_{i}, \gamma_{2}(G)\right\rangle$ and the generators of the commutator subgroups $\gamma_{2}\left(M_{i}\right), 1 \leq i \leq 4$, modulo $\gamma_{4}(G)$ for each of the isomorphism classes of groups $G$ with $m \geq 5$, $n \geq 6, e=3$. Several of these classes have the same principalization type and the same parameters $\beta, \delta$. The left number of isomorphism classes concerns the single case $m=5$ [37, pp. 8-9], the right number odd values of $m \geq 7$ [37, pp. 8-9 and pp. 16-19] and the middle number even values of $m \geq 6$ [37, pp. $10-12$ and pp. $13-15]$.

TABLE 7. Parameters, third powers, and generators for $m \geq 5, n \geq 6, e=3$

\begin{tabular}{|l|ccc|cc|cccc|cccc|}
\hline Type & isom. cl. & $\beta$ & $\delta$ & $y^{3}$ & $x^{3}$ & $\overline{(x y)^{3}}$ & $\overline{\left(x y^{-1}\right)^{3}}$ & $\overline{t_{3}}$ & $\overline{s_{3}}$ & $\overline{s_{3} t_{3}}$ & $s_{3} t_{3}^{-1}$ \\
\hline b.10 & 1 & 7 & 9 & 0 & 0 & $\sigma_{3}$ & $\tau_{3}$ & 1 & 1 & $\tau_{3}$ & $\sigma_{3}^{-1}$ & $\sigma_{3}^{-1} \tau_{3}$ & $\sigma_{3}^{-1} \tau_{3}^{-1}$ \\
d.19 & 1 & 2 & 1 & 0 & 0 & $\sigma_{3}$ & $\tau_{3}$ & 1 & 1 & $\tau_{3}$ & $\sigma_{3}^{-1}$ & $\sigma_{3}^{-1} \tau_{3}$ & $\sigma_{3}^{-1} \tau_{3}^{-1}$ \\
d.23 & 1 & 1 & 1 & 0 & 0 & $\sigma_{3}$ & $\tau_{3}$ & 1 & 1 & $\tau_{3}$ & $\sigma_{3}^{-1}$ & $\sigma_{3}^{-1} \tau_{3}$ & $\sigma_{3}^{-1} \tau_{3}^{-1}$ \\
d.25 & 1 & 2 & 1 & 0 & 0 & $\sigma_{3}$ & $\tau_{3}$ & 1 & 1 & $\tau_{3}$ & $\sigma_{3}^{-1}$ & $\sigma_{3}^{-1} \tau_{3}$ & $\sigma_{3}^{-1} \tau_{3}^{-1}$ \\
\hline c.18 & 1 & 1 & 1 & -1 & 1 & $\sigma_{3}$ & $\tau_{3}$ & 1 & $\tau_{3}^{-2}$ & $\tau_{3}^{2}$ & $\sigma_{3}^{-1} \tau_{3}$ & $\sigma_{3}^{-1}$ & $\sigma_{3}^{-1} \tau_{3}^{-1}$ \\
E.6 & 1 & 1 & 1 & -1 & 1 & $\sigma_{3}$ & $\tau_{3}$ & 1 & $\tau_{3}^{-2}$ & $\tau_{3}^{2}$ & $\sigma_{3}^{-1} \tau_{3}$ & $\sigma_{3}^{-1}$ & $\sigma_{3}^{-1} \tau_{3}^{-1}$ \\
E.14 & 1 & 2 & 1 & -1 & 1 & $\sigma_{3}$ & $\tau_{3}$ & 1 & $\tau_{3}^{-2}$ & $\tau_{3}^{2}$ & $\sigma_{3}^{-1} \tau_{3}$ & $\sigma_{3}^{-1}$ & $\sigma_{3}^{-1} \tau_{3}^{-1}$ \\
H.4 & 1 & 8 & 9 & -1 & 1 & $\sigma_{3}$ & $\tau_{3}$ & 1 & $\tau_{3}^{-2}$ & $\tau_{3}^{2}$ & $\sigma_{3}^{-1} \tau_{3}$ & $\sigma_{3}^{-1}$ & $\sigma_{3}^{-1} \tau_{3}^{-1}$ \\
\hline c.21 & 1 & 1 & 1 & 0 & 1 & $\sigma_{3}$ & $\tau_{3}$ & $\tau_{3}$ & $\tau_{3}^{-1}$ & $\tau_{3}$ & $\sigma_{3}^{-1} \tau_{3}$ & $\sigma_{3}^{-1} \tau_{3}^{2}$ & $\sigma_{3}^{-1}$ \\
E.8 & 1 & 1 & 1 & 0 & 1 & $\sigma_{3}$ & $\tau_{3}$ & $\tau_{3}$ & $\tau_{3}^{-1}$ & $\tau_{3}$ & $\sigma_{3}^{-1} \tau_{3}$ & $\sigma_{3}^{-1} \tau_{3}^{2}$ & $\sigma_{3}^{-1}$ \\
E.9 & 1 & 2 & 1 & 0 & 1 & $\sigma_{3}$ & $\tau_{3}$ & $\tau_{3}$ & $\tau_{3}^{-1}$ & $\tau_{3}$ & $\sigma_{3}^{-1} \tau_{3}$ & $\sigma_{3}^{-1} \tau_{3}^{2}$ & $\sigma_{3}^{-1}$ \\
G.16 & 1 & 8 & 9 & 0 & 1 & $\sigma_{3}$ & $\tau_{3}$ & $\tau_{3}$ & $\tau_{3}^{-1}$ & $\tau_{3}$ & $\sigma_{3}^{-1} \tau_{3}$ & $\sigma_{3}^{-1} \tau_{3}^{2}$ & $\sigma_{3}^{-1}$ \\
\hline
\end{tabular}

The order of the coset of $g_{i} \in M_{i}$ with respect to $\gamma_{2}\left(M_{i}\right)$ is bounded from above by 3 if and only if the third power $g_{i}^{3}$ is contained in $\gamma_{2}\left(M_{i}\right)$. 
Theorem 4.4. (TTT $\tau(G)$ of groups $G$ on coclass trees of $\mathcal{G}(3,2)$ )

Let $K$ be a number field with 3 -class group $\mathrm{Cl}_{3}(K)$ of type $(3,3)$. Suppose that the second 3 -class group $G=\operatorname{Gal}\left(\mathrm{F}_{3}^{2}(K) \mid K\right)$ of $K$ is of order $|G|=3^{n}$ and of class $\mathrm{cl}(G)=m-1$, where $n \geq 6$ and $m=n-1$, $i$. e., that $G$ is a vertex on one of the three coclass trees of coclass graph $\mathcal{G}(3,2)$ in Figure 2, with invariant $e=3$. In the case $m=5, n=6$ let $\left[\chi_{s}(G), \gamma_{e}(G)\right]=1$.

Then the structure of the 3-class groups $\mathrm{Cl}_{3}\left(N_{i}\right)$ of the four unramified cyclic cubic extensions $N_{i}$ of $K$ is given by Table 8 , in dependence on the principalization type $\varkappa$ of $K$. The invariant $\varepsilon$ denotes the number of 3-class groups $\mathrm{Cl}_{3}\left(N_{i}\right)$ of type $(3,3,3)$, for each principalization type. Generally, the first two 3-class groups $\mathrm{Cl}_{3}\left(N_{1}\right)$ and $\mathrm{Cl}_{3}\left(N_{2}\right)$ are nearly homocyclic.

TABLE 8. 3-class groups of type $(3,3,3)$ for $m \geq 5, n \geq 6, e=3$

\begin{tabular}{|lc|cccc|c|}
\hline Type & $\varkappa$ & $\mathrm{Cl}_{3}\left(N_{1}\right)$ & $\mathrm{Cl}_{3}\left(N_{2}\right)$ & $\mathrm{Cl}_{3}\left(N_{3}\right)$ & $\mathrm{Cl}_{3}\left(N_{4}\right)$ & $\varepsilon$ \\
\hline b.10 & $(0043)$ & $\mathrm{A}(3, m-1)$ or A(3, $m-2)$ & $(9,3)$ & $(3,3,3)$ & $(3,3,3)$ & 2 \\
d.19 & $(4043)$ & $\mathrm{A}(3, m-1)$ & $(9,3)$ & $(3,3,3)$ & $(3,3,3)$ & 2 \\
d.23 & $(1043)$ & $\mathrm{A}(3, m-1)$ & $(9,3)$ & $(3,3,3)$ & $(3,3,3)$ & 2 \\
d.25 & $(2043)$ & $\mathrm{A}(3, m-1)$ & $(9,3)$ & $(3,3,3)$ & $(3,3,3)$ & 2 \\
\hline c.18 & $(0313)$ & $\mathrm{A}(3, m-1)$ & $(9,3)$ & $(3,3,3)$ & $(9,3)$ & 1 \\
E.6 & $(1313)$ & $\mathrm{A}(3, m-1)$ & $(9,3)$ & $(3,3,3)$ & $(9,3)$ & 1 \\
E.14 & $(2313)$ & $\mathrm{A}(3, m-1)$ & $(9,3)$ & $(3,3,3)$ & $(9,3)$ & 1 \\
H.4 & $(3313)$ & $\mathrm{A}(3, m-1)$ or A(3,m-2) & $(9,3)$ & $(3,3,3)$ & $(9,3)$ & 1 \\
\hline c.21 & $(0231)$ & $\mathrm{A}(3, m-1)$ & $(9,3)$ & $(9,3)$ & $(9,3)$ & 0 \\
E.8 & $(1231)$ & $\mathrm{A}(3, m-1)$ & $(9,3)$ & $(9,3)$ & $(9,3)$ & 0 \\
E.9 & $(2231)$ & $\mathrm{A}(3, m-1)$ & $(9,3)$ & $(9,3)$ & $(9,3)$ & 0 \\
G.16 & $(4231)$ & $\mathrm{A}(3, m-1)$ or A $(3, m-2)$ & $(9,3)$ & $(9,3)$ & $(9,3)$ & 0 \\
\hline
\end{tabular}

Proof. The structure of the first 3-class group $\mathrm{Cl}_{3}\left(N_{1}\right)$ is given here only for the sake of completeness and is contained in the statement of Theorem 3.2 already.

Similarly as in the proof of Theorem 4.2, the structure of the other 3-class groups $\mathrm{Cl}_{3}\left(N_{i}\right) \simeq$ $M_{i} / \gamma_{2}\left(M_{i}\right)$ with $2 \leq i \leq 4$ is a consequence of table 7 , when the preparations in the $\S \S 4.2 .24 .2 .3$, resp. in Lemma 4.1, are taken into consideration.

Corollary 4.4.1. (E as a tree invariant)

All metabelian groups $G$ on the coclass tree $\mathcal{T}(\langle 729,40\rangle)$, resp. $\mathcal{T}(\langle 243,6\rangle)$, resp. $\mathcal{T}(\langle 243,8\rangle)$, of coclass graph $\mathcal{G}(3,2)$ in Figure 2 are characterized by the value $\varepsilon=2$, resp. $\varepsilon=1$, resp. $\varepsilon=0$.

Proof. This is a consequence of Theorem 4.4 and the diagram [36, p. 189 ff.]. See also [33, Thm. $3.16-3.17]$.

Corollary 4.4.2. As before, for these isomorphism classes of the second 3-class group $G$, the extensions with 3-class group of type $(3,3,3)$ satisfy the condition (B) of Taussky [42, $i . \quad e$. , they have a partial principalization without fixed point. However, here only the following weaker statement without admissible inversion is true: if a 3-class group $\mathrm{Cl}_{3}\left(N_{i}\right), 3 \leq i \leq 4$, is of type $(3,3,3)$, then the norm class group $\operatorname{Norm}_{N_{i} \mid K}\left(\mathrm{Cl}_{3}\left(N_{i}\right)\right)$ becomes principal in either two or three of the extensions $N_{\ell}, 1 \leq \ell \leq 4$.

Proof. This follows by evaluating the principalization type $\varkappa$ of the isomorphism classes.

Corollary 4.4.3. If $K$ is a quadratic base field with $G$ on a coclass tree of $\mathcal{G}(3,2)$, then the four total principalization types b.10, d.19, d.23, d.25 with $\varepsilon=2$, i. e., $G \in \mathcal{T}(\langle 729,40\rangle)$, are impossible, due to class number relations. The remaining eight principalization types cannot be determined uniquely by the invariant $\varepsilon$ alone.

(1) The total principalization types c.18 with $\varepsilon=1$ and c.21 with $\varepsilon=0$ are characterized by an even 3-exponent $u$ of the first 3 -class number $\mathrm{h}_{3}\left(N_{1}\right)=3^{u}$. 
(2) The partial principalization types E.6, E.14 with $\varepsilon=1$ and E.8, E.9 with $\varepsilon=0$ are determined by an odd 3-exponent $w$ of the 3 -class number $\mathrm{h}_{3}\left(\mathrm{~F}_{3}^{1}(K)\right)=3^{w}$.

(3) The partial principalization types $\mathrm{H} .4$ with $\varepsilon=1$ and G.16 with $\varepsilon=0$ are characterized by an even 3-exponent $w$ of the 3 -class number $\mathrm{h}_{3}\left(\mathrm{~F}_{3}^{1}(K)\right)=3^{w}$, provided the weak leaf conjecture 4.2 holds.

Here, as before, $\mathrm{F}_{3}^{1}(K)$ denotes the first Hilbert 3-class field of $K$.

Proof. For the principalization types b.10, d.19, d.23, and d.25 having $\varkappa(2)=0$, the entire 3 -class group $\mathrm{Cl}_{3}(K)$ becomes principal in $N_{2}$. Therefore a quadratic base field $K$ must be real and the unramified cyclic cubic extension $N_{2}$ must be an $S_{3}$-field of type $\alpha$ with even 3-exponent of the 3-class number $\mathrm{h}_{3}\left(N_{2}\right)$, in contradiction to $\mathrm{Cl}_{3}\left(N_{2}\right) \simeq \mathrm{A}(3,3)$. See [30, Prop. 4.3-4.4, p. 484-485].

The parity of the 3 -exponent of the first 3-class number $\mathrm{h}_{3}\left(N_{1}\right)=3^{u}$ turns out to be even, $u=m-1 \equiv 0(\bmod 2)$, for the total principalization types c.18 and c.21 with $\varkappa(1)=0$, to be odd, $u=m-1 \equiv 1(\bmod 2)$, for the partial principalization types E.6, E.14 and E.8, E.9 with $k=0$ and thus $w=n-2$ odd, and to be odd, $u=m-2 \equiv 1(\bmod 2)$, for the partial principalization types H.4 and G.16 with conjectural $k=1$ (see Conjecture 4.2) and thus $w=n-2$ even.

Here, we use the relation $3^{w}=\mathrm{h}_{3}\left(\mathrm{~F}_{3}^{1}(K)\right)=\left|\gamma_{2}(G)\right|=3^{n-2}$ and [30, Thm. 5.2-5.3, p. 492493].

Example 4.4. The first occurrences of second 3-class groups $G=\operatorname{Gal}\left(\mathrm{F}_{3}^{2}(K) \mid K\right)$ on the coclass trees $\mathcal{T}(\langle 243,6\rangle)$ and $\mathcal{T}(\langle 243,8\rangle)$ with invariants $m \geq 5, n \geq 6, e=3$ and bicyclic center, $k=0$, resp. cyclic center, $k=1$, over quadratic base fields $K=\mathbb{Q}(\sqrt{D})$ with 3-class group of type $(3,3)$ and discriminant $-10^{6}<D<0$, resp. $0<D<10^{7}$, are summarised in Table 9, Here, $|D|$ denotes the smallest absolute value of the discriminant of a complex quadratic field $K$, and $D$ the smallest discriminant of a real quadratic field $K$, of the corresponding principalization type. The earlier computations by Scholz and Taussky [40, Heider and Schmithals [22, and Brink [11, are confirmed. Cases without references, in particular all cases with real quadratic base fields, were unknown up to now.

TABLE 9. Examples for groups $G$ on coclass trees with $m \geq 5, n \geq 6, e=3$

\begin{tabular}{|c|c|c|c|c|c|c|c|}
\hline Type & $\varkappa$ & $m$ & $n$ & $k$ & $|D|$ & ref. & $\bar{D}$ \\
\hline $\begin{array}{ll}\text { c. } 18 \\
\end{array}$ & $(0313)$ & 5 & 6 & 0 & impossible & & 534824 \\
\hline E. 6 & (1313) & 6 & 7 & 0 & 15544 & 22 & 5264069 \\
\hline E. $6 \uparrow$ & (1313) & 8 & 9 & 0 & 268040 & & unknown \\
\hline E.14 & (2313) & 6 & 7 & 0 & 16627 & 22 & 3918837 \\
\hline E. $14 \uparrow$ & (2313) & 8 & 9 & 0 & 262744 & & unknown \\
\hline $\mathrm{H} .4 \uparrow$ & (3313) & 7 & 8 & 1 & 21668 & {$[11,28$} & 1162949 \\
\hline H. $4 \uparrow^{2}$ & (3313) & 9 & 10 & 1 & 446788 & & unknown \\
\hline c.21 & $\overline{(0231)}$ & 5 & 6 & 0 & impossible & & 540365 \\
\hline c. $21 \uparrow$ & (0231) & 7 & 8 & 0 & impossible & & 1001957 \\
\hline E.8 & (1231) & 6 & 7 & 0 & 34867 & & 6098360 \\
\hline E. $8 \uparrow$ & (1231) & 8 & 9 & 0 & 370740 & & unknown \\
\hline E.9 & (2231) & 6 & 7 & 0 & 9748 & 40] & 342664 \\
\hline E. $9 \uparrow$ & (2231) & 8 & 9 & 0 & 297079 & & unknown \\
\hline G.16 & $(4231)$ & 7 & 8 & 1 & 17131 & 22 & 8711453 \\
\hline G. $16 \uparrow$ & (4231) & 9 & 10 & 1 & 819743 & & unknown \\
\hline
\end{tabular}

Whereas the parameters $m=4, n=5$ for the principalization types D.10, D.5 in example 4.2 and the parameters $m=5, n=6$ for the ground state of principalization types G.19, H.4 in example 4.3, are determined uniquely, we now have infinite coclass families of second 3-class groups $G=\operatorname{Gal}\left(\mathrm{F}_{3}^{2}(K) \mid K\right)$ with strictly increasing nilpotency class $\operatorname{cl}(G)=m-1$, sharing the same principalization type. For this reason, we define excited states of principalization types, denoted by arrows $\uparrow, \uparrow^{2}$, and so on. The index $m$ of nilpotency can take all odd values $m \geq 5$ for 
the principalization types c.18, c.21, all even values $m \geq 6$ for the principalization types E.6, E.14, E.8, E.9, and all odd values $m \geq 7$ for principalization types H.4, G.16.

A special feature of the principalization types c.18 and c.21 is the location of their second 3-class groups $G=\operatorname{Gal}\left(\mathrm{F}_{3}^{2}(K) \mid K\right)$ as infinitely capable vertices on mainlines of coclass trees $\mathcal{T}(\langle 243,6\rangle)$ and $\mathcal{T}(\langle 243,8\rangle)$.

Concrete numerical realisations are known for the ground state with minimal index of nilpotency $m$, for each of these infinite coclass families. Realisations for excited states with higher values of $m$ are known for the principalization types E.6, E.14, E.8, E.9, H.4, and G.16 of complex quadratic fields and for the principalization type c.21 of real quadratic fields.

4.6. Groups $G$ of coclass $\operatorname{cc}(G) \geq 3$ with $m \geq 5, n \geq 7$. This section corresponds to the fifth and sixth row of Table 1. Here we must investigate the abelianizations of only two maximal subgroups $M_{i}, 3 \leq i \leq 4$. The third powers of the generators of these groups satisfy the following general relations, according to [36, Lem. 3.4.11, p. 105].

$$
\begin{aligned}
(x y)^{3} & =\sigma_{m-2}^{\rho(\beta+\delta)} \sigma_{m-1}^{\alpha+\gamma+\rho(\beta+\delta)} \tau_{e}^{\beta+\delta} \\
\left(x y^{-1}\right)^{3} & =\sigma_{m-2}^{\rho(\delta-\beta)} \sigma_{m-1}^{\alpha-\gamma+\rho \beta} \tau_{e}^{\beta-\delta} .
\end{aligned}
$$

Therefore the order of the coset of $x y \in M_{3}$ with respect to $\gamma_{2}\left(M_{3}\right)$ and of the coset of $x y^{-1} \in M_{4}$ with respect to $\gamma_{2}\left(M_{4}\right)$ is certainly bounded from above by 3 , when $m \geq 6$ and $e \geq 4$, and thus $\sigma_{m-2}, \sigma_{m-1}, \tau_{e} \in \gamma_{4}(G)<\gamma_{2}\left(M_{i}\right)$, for $3 \leq i \leq 4$.

It remains to investigate the 15 isomorphism classes of groups with $m=5, n=7, e=4$, for which $\left[\chi_{s}(G), \gamma_{e}(G)\right]=1$ and thus $\rho=0$ [37, pp. 34-35]. For these isomorphism classes, third powers of the generators are given by

$$
\begin{aligned}
(x y)^{3} & =\sigma_{4}^{\alpha+\gamma} \tau_{4}^{\beta+\delta}, \\
\left(x y^{-1}\right)^{3} & =\sigma_{4}^{\alpha-\gamma} \tau_{4}^{\beta-\delta}
\end{aligned}
$$

and therefore ord $(\overline{x y}) \leq 3$ and $\operatorname{ord}\left(\overline{x y^{-1}}\right) \leq 3$, independently from all parameters $\alpha, \beta, \gamma, \delta$, since $\sigma_{4}, \tau_{4} \in \gamma_{4}(G)<\gamma_{2}\left(M_{i}\right)$, for $3 \leq i \leq 4$.

Theorem 4.5. Let $K$ be a number field with 3-classgroup $\mathrm{Cl}_{3}(K)$ of type $(3,3)$ and with second 3 -class group $G=\operatorname{Gal}\left(\mathrm{F}_{3}^{2}(K) \mid K\right)$ of order $|G|=3^{n}, n \geq 7$, and of coclass $\operatorname{cc}(G) \geq 3, m \leq n-2$, $e \geq 4$.

Then the structure of the 3-class groups of the four unramified cyclic cubic extensions $N_{i}$ of $K$ is nearly homocyclic for $\mathrm{Cl}_{3}\left(N_{1}\right), \mathrm{Cl}_{3}\left(N_{2}\right)$, and elementary abelian of type $(3,3,3)$ for $\mathrm{Cl}_{3}\left(N_{3}\right)$, $\mathrm{Cl}_{3}\left(N_{4}\right)$, independently from the principalization type $\varkappa$ of $K$. The number of 3-class groups $\mathrm{Cl}_{3}\left(N_{i}\right)$ of type $(3,3,3)$ is always given by $\varepsilon=2$. The extensions with 3-class group of type $(3,3,3)$ satisfy Taussky's condition (B) [42, that is, they have a partial principalization without fixed point.

Proof. The nearly homocyclic structure of the first and second 3-class group, $\mathrm{Cl}_{3}\left(N_{1}\right), \mathrm{Cl}_{3}\left(N_{2}\right)$, is contained in the statement of Theorem 3.2 already.

Similarly as in the proof of Theorem 4.2, the elementary abelian structure of the third and fourth 3-class group, $\mathrm{Cl}_{3}\left(N_{i}\right) \simeq M_{i} / \gamma_{2}\left(M_{i}\right), 3 \leq i \leq 4$, is a consequence of the considerations at the begin of this section, if we take into account the preparation in $\S 4.2 .3$.

Example 4.5. The first occurrences of second 3-class groups $G=\operatorname{Gal}\left(\mathrm{F}_{3}^{2}(K) \mid K\right)$ of $\operatorname{coclass} \operatorname{cc}(G) \geq$ 3 with invariants $m \geq 6, n \geq 8, e \geq 4$, and bicyclic center, $k=0$, resp. cyclic center, $k=1$, over quadratic fields $K=\mathbb{Q}(\sqrt{D})$ with 3 -class group of type $(3,3)$ and discriminant $-10^{6}<D<0$, resp. $0<D<10^{7}$, are summarized in Table [10. Here, $|D|$ denotes the smallest absolute value of the discriminant of a complex quadratic field $K$ and $D$ the smallest discriminant of a real quadratic field $K$ of the corresponding principalization type. The earlier computations by Brink [11] are confirmed. 
TABLE 10. Examples for groups $G$ with $\operatorname{cc}(G) \geq 3, m \geq 6, n \geq 8, e \geq 4$

\begin{tabular}{|c|c|c|c|c|c|c|c|c|c|}
\hline Type & $\varkappa$ & $m$ & $n$ & $e$ & $k$ & $\mathrm{Cl}_{3}\left(\mathrm{~F}_{3}^{1}(K)\right)$ & $|D|$ & ref. & $D$ \\
\hline b.10 & $(0043)$ & 6 & 8 & 4 & 1 & $(9,9,3,3)$ & impossible & & 710652 \\
\hline $\mathrm{d} .19$ & $(4043)$ & 6 & 8 & 4 & 0 & $(9,9,3,3)$ & impossible & & 2328721 \\
\hline d. 23 & (1043) & 6 & 8 & 4 & 0 & $(9,9,3,3)$ & impossible & & 1535117 \\
\hline $\mathrm{d} .25^{*}$ & (0143) & 7 & 10 & 5 & 0 & $(27,9,9,3)$ & impossible & & 8491713 \\
\hline F.7 & $(3443)$ & 6 & 9 & 5 & 0 & $(9,9,9,3)$ & 124363 & & unknown \\
\hline F.7个 & $(3443)$ & 8 & 11 & 5 & 0 & $(27,27,9,3)$ & 469816 & & unknown \\
\hline F.11 & (1143) & 6 & 9 & 5 & 0 & $(9,9,9,3)$ & 27156 & [11, 28] & unknown \\
\hline F.11个 & (1143) & 8 & 11 & 5 & 0 & $(27,27,9,3)$ & 469787 & & unknown \\
\hline F.12 & (1343) & 6 & 9 & 5 & 0 & $(9,9,9,3)$ & 31908 & 11 & unknown \\
\hline F.12个 & $(1343)$ & 8 & 11 & 5 & 0 & $(27,27,9,3)$ & 249371 & & unknown \\
\hline F. $12 \uparrow^{2}$ & (1343) & 8 & 13 & 7 & 0 & $(27,27,27,9)$ & 423640 & & unknown \\
\hline F.13 & $(3143)$ & 6 & 9 & 5 & 0 & $(9,9,9,3)$ & 67480 & 11 & 8321505 \\
\hline F.13个 & $(3143)$ & 8 & 11 & 5 & 0 & $(27,27,9,3)$ & 159208 & & 8127208 \\
\hline G.16r & $(1243)$ & 7 & 10 & 5 & 1 & $(27,9,9,3)$ & 290703 & & unknown \\
\hline G.16i & (1243) & 7 & 10 & 5 & 1 & $(9,9,9,9)$ & 135059 & & unknown \\
\hline G.19r & $(2143)$ & 7 & 10 & 5 & 1 & $(27,9,9,3)$ & 96827 & & unknown \\
\hline G.19r $\uparrow$ & $(2143)$ & 9 & 12 & 5 & 1 & $(81,27,9,3)$ & 509160 & & unknown \\
\hline G.19i & $(2143)$ & 7 & 10 & 5 & 1 & $(9,9,9,9)$ & 199735 & & unknown \\
\hline H.4r & $(3343)$ & 7 & 10 & 5 & 1 & $(27,9,9,3)$ & 256935 & & unknown \\
\hline $\mathrm{H} .4 \mathrm{r} \uparrow$ & $(3343)$ & 9 & 12 & 5 & 1 & $(81,27,9,3)$ & 678804 & & unknown \\
\hline H. $4 \mathrm{i}$ & $(3343)$ & 7 & 10 & 5 & 1 & $(9,9,9,9)$ & 186483 & & unknown \\
\hline
\end{tabular}

Similarly as in example 4.4 these principalization types belong to infinite families of second 3-class groups $G=\operatorname{Gal}\left(\mathrm{F}_{3}^{2}(K) \mid K\right)$. Here, additionally to the nilpotency class $\operatorname{cl}(G)=m-1$, the coclass $\operatorname{cc}(G)=e-1=n-m+1$ can also take infinitely many values.

For the principalization types F.7, F.11, F.12, F.13 with defect $k=0$, all even values $m \geq 6$ and all odd values $5 \leq e \leq m-1$ are possible.

The very rare principalization types d.19, d.23, d.25, also having $k=0$, play a unique exceptional role [31, Thm. 3.4, p. 491], since their second 3-class groups $G=\operatorname{Gal}\left(\mathrm{F}_{3}^{2}(K) \mid K\right)$ can appear either as terminal metabelian vertices (leaves) with even values $m \geq 6$ and even values $4 \leq e \leq m-1$ or as infinitely capable vertices on mainlines of coclass trees with odd values $m \geq 7$ and odd values $5 \leq e \leq m-1$.

Assuming the weak leaf conjecture 4.2, the following types show up with defect $k=1$ only. For the principalization type b.10 all even values $m \geq 6$ and all even values $4 \leq e \leq m-2$ can occur, and for the principalization types G.16, G.19, and H.4 all odd values $m \geq 7$ and all odd values $5 \leq e \leq m-2$ are admissible.

Concrete numerical realizations by complex quadratic fields are known for the ground state of these principalization types with minimal index of nilpotency $m$, but only partially by real quadratic base fields.

\section{IMPLEMENTING THE PRINCIPALIZATION ALGORITHM FOR QUADRATIC FIELDS}

In this section we describe the computational techniques used to achieve the numerical results presented in $\S$ 6. The new principalization algorithm via class group structure has been implemented for quadratic fields, having a 3-class group of type $(3,3)$, with the aid of program scripts written for the number theoretical computer algebra system PARI/GP [7, 38. We refer to the relevant methods of this software package by printing their names in typewriter font with trailing parentheses.

\subsection{Generating polynomials for non-Galois cubic fields $L$.}


5.1.1. Simply real cubic fields. Generating polynomials $p(X)=X^{3}-b X^{2}+c X-d$ of third degree for simply real cubic fields $L$ of signature $(1,1)$ are obtained in the following manner. Suppose the intended upper bound for the absolute value of the field discriminant is $|\mathrm{d}(L)| \leq U$. Then candidates for the coefficients $b, c$, and $d$ run over three nested loops $1 \leq b \leq\lfloor 3+2 \sqrt[4]{U}\rfloor$, $1 \leq c \leq\left\lfloor\frac{b^{2}+\sqrt{U}}{3}\right\rfloor$, and $1 \leq d \leq\left\lfloor\frac{b^{2}-3+2 \sqrt{U}}{6}\right\rfloor$, with bounds due to Godwin and Angell, given by Fung and Williams [18, (2.5), p. 315]. For each triplet $(b, c, d)$ the following tests are performed.

(1) Reducible polynomials $p(X)$ are eliminated with the aid of polisirreducible().

(2) For irreducible polynomials the discriminant $\mathrm{d}(L)$ of the cubic field $L=\mathbb{Q}(\vartheta)$, generated by the real zero $\vartheta$ of $p(X)$, is calculated by means of $\operatorname{nfdisc}()$ and tested for $-U \leq \mathrm{d}(L)<0$.

(3) By poldisc() the discriminant $\mathrm{d}(p)$ of the polynomial is computed and its index $\mathrm{i}(p)$ is determined, using the formula $\mathrm{d}(p)=\mathrm{i}(p)^{2} \cdot \mathrm{d}(L)$. Polynomials with indices bigger than the bound

$$
\frac{\sqrt{124 b^{2}+432 b+4 \sqrt{U}+729}}{3 \sqrt{3}}
$$

are skipped, thus discouraging superfluous isomorphic fields [18, (2.6), p. 315].

(4) Field discriminants $\mathrm{d}(L)=f^{2} \cdot \mathrm{d}(K)$ [32, $\S 1$, p. 832] are restricted to fundamental discriminants with conductor $f=1$ by isfundamental().

(5) Finally, the 3-class group $\mathrm{Cl}_{3}(K)$ of the complex quadratic subfield $K$ of the Galois closure $N$ of $L$, which is unramified with conductor $f=1$ over $K$ and has discriminant $\mathrm{d}(N)=\mathrm{d}(K)^{3}$ [32, Abstract, p. 831], is restricted to the type $(3,3)$ with the aid of quadclassunit(), thereby eliminating the numerous cyclic 3-class groups.

5.1.2. Totally real cubic fields. Trace free generating polynomials $p(X)=X^{3}-c X-d$ for totally real cubic fields $L$ of signature $(3,0)$ are collected in the following way. If the desired upper bound for the field discriminant is $\mathrm{d}(L) \leq U$, then candidates for the coefficients $c$ and $d$ run over two nested loops $1 \leq c \leq\lfloor\sqrt{U}\rfloor$ and $1 \leq d \leq\left\lfloor\sqrt{\frac{4 c^{3}}{27}}\right\rfloor$, with bounds given by Llorente and Quer [27, $\S 3$, p. 586]. For each pair $(c, d)$ the following tests are performed.

(1) Reducible polynomials $p(X)$ are skipped with the aid of polisirreducible().

(2) For irreducible polynomials the discriminant $\mathrm{d}(L)$ of the cubic field $L=\mathbb{Q}(\xi)$, generated by a zero $\xi$ of $p(X)$, is calculated by means of nfdisc() and checked for $0<\mathrm{d}(L) \leq U$.

(3) A further bound [27, Thm. 3, p. 584] is imposed on the linear coefficient

$$
c \leq \begin{cases}\left\lfloor\frac{\sqrt{\mathrm{d}(L)}}{3}\right\rfloor, & \text { if } 27 \mid \mathrm{d}(L), \\ \lfloor\sqrt{\mathrm{d}(L)}\rfloor, & \text { otherwise. }\end{cases}
$$

(4) By poldisc () the discriminant $\mathrm{d}(p)$ of the polynomial is computed and its index $\mathrm{i}(p)$ is determined, using the formula $\mathrm{d}(p)=\mathrm{i}(p)^{2} \cdot \mathrm{d}(L)$. Polynomials with index bigger than the bound

$$
\begin{cases}\left\lfloor 2 \sqrt{\frac{c}{3}}\right\rfloor, & \text { if } 27 \mid \mathrm{d}(L), \\ \lfloor 2 \sqrt{c}\rfloor, & \text { otherwise, }\end{cases}
$$

are eliminated [27, Thm. 3, p. 584].

(5) Field discriminants $\mathrm{d}(L)$ are restricted to fundamental discriminants by isfundamental ().

(6) Finally, the 3-class group $\mathrm{Cl}_{3}(K)$ of the real quadratic subfield $K$ of the Galois closure $N$ of $L$ is restricted to the type $(3,3)$ with the aid of quadclassunit().

5.2. Structure of 3-class groups $\mathrm{Cl}_{3}(N)$ of $S_{3}$-fields $N$. The generating polynomials of $\S 5.1$ are stored as quadruplets $(D, b, c, d)$ for $D<0$, ordered by descending discriminants $D$, resp. as triplets $(D, c, d)$ for $D>0$, ordered by ascending discriminants $D$. The bounds in [18, resp. [27], provide a warranty that, for each discriminant $D=\mathrm{d}(K)=\mathrm{d}(L)$, generating polynomials for all four non-isomorphic cubic fields $L$ sharing the same discriminant $\mathrm{d}(L)=D$ [32, Cor. 3.1, p. 838] are contained in the list. Now the polynomials are iterated through the list in a single loop and for each of them the following steps are executed. 
(1) The regulator $\mathrm{R}(L)$ and the class number $\mathrm{h}(L)$ of $L$ are calculated with the aid of bnfinit() using the flag 1 , which ensures that a fundamental system of units of $L$ is determined. An indicator is stored, if the class number $\mathrm{h}(L)$ is divisible by 9 or 27 .

(2) By means of polcompositum(), applied to the cubic polynomial $p(X)=X^{3}-b X^{2}+c X-d$, resp. $p(X)=X^{3}-c X-d$, and the quadratic polynomial $q(X)=X^{2}-D$, a generating polynomial $s(X)$ of sixth degree for the Galois closure $N$ of $L$ is calculated.

(3) The polynomial $s(X)$ of sixth degree is used to determine the structure of the class group $\mathrm{Cl}(N)$ of the normal field $N$ with the aid of bnfinit(), where the flag is set to 1 . An indicator is stored, if the first three abelian type invariants $\left(n_{1}, n_{2}, n_{3}, \ldots\right)$ of the class group structure are all divisible by 3 , that is, if the 3 -class group $\mathrm{Cl}_{3}(N)$ of $N$ is elementary abelian of type $(3,3,3)$.

The results are evaluated in the following way. The structures of the 3-class groups $\mathrm{Cl}_{3}\left(N_{i}\right)$, $1 \leq i \leq 4$, of quadruplets $N_{1}, \ldots, N_{4}$ of $S_{3}$-fields sharing the same discriminant $D^{3}$, form the transfer target type $\tau(K)$ and determine the number $\varepsilon(K)$ of groups of type $(3,3,3)$. If some 3 -class group $\mathrm{Cl}_{3}\left(N_{i}\right)$ is of type $(3,3)$, that is, isomorphic to $\mathrm{A}(3,2)$, then the second 3-class group $G$ of $K$ is of coclass cc $(G)=1$, by Theorem 3.1, and the algorithm can be terminated. Otherwise $G$ is of coclass $\operatorname{cc}(G) \geq 2$ and the algorithm can be terminated only if no indicator of $9 \mid \mathrm{h}\left(L_{i}\right)$ has been stored, for any $1 \leq i \leq 4$. The transfer target type $\tau(K)$ and the invariant $\varepsilon(K)$ determine the principalization type (transfer kernel type) $\varkappa(K)$ and the structure of the second 3-class group $G=\mathrm{G}_{3}^{2}(K)=\operatorname{Gal}\left(\mathrm{F}_{3}^{2}(K) \mid K\right)$ of the quadratic field $K$ in two important special cases: uniquely for sporadic $G$ of coclass $\operatorname{cc}(G)=2$, according to Theorems 4.2 and 4.3, and up to separation of types a. 2 and a.3 for $\operatorname{cc}(G)=1$, according to Theorem 4.1 .

5.3. First Hilbert 3-class field $\mathrm{F}_{3}^{1}(K)$ of $K$. For the quadruplets $L_{1}, \ldots, L_{4}$ of cubic fields, sharing the same discriminant $D$, which have been marked by an indicator of $9 \mid \mathrm{h}\left(L_{i}\right)$, for some $1 \leq i \leq 4$, a further step must be appended, when the second 3-class group $G=\operatorname{Gal}\left(\mathrm{F}_{3}^{2}(K) \mid K\right)$ of the quadratic field $K$ is of coclass $\operatorname{cc}(G) \geq 2$. A generating polynomial $f(X)$ of eighteenth degree for the first Hilbert 3-class field $\mathrm{F}_{3}^{1}(K)=N_{i} \cdot L_{j}$ of $K$ is calculated by means of polcompositum(), applied to the generating polynomial $s(X)$ of sixth degree of $N_{i}$ and the cubic generating polynomial $p(X)$ of $L_{j}$, for some $1 \leq i \neq j \leq 4$. The polynomial $f(X)$ of eighteenth degree is used to determine the structure of the class group $\mathrm{Cl}\left(\mathrm{F}_{3}^{1}(K)\right)$ of the first Hilbert 3-class field $\mathrm{F}_{3}^{1}(K)$ of the quadratic field $K$ with the aid of bnfinit(), where the flag is set to 1 . Whereas computations for fields of third and sixth degree are usually a matter of less than a second in PARI/GP, the CPU time for a field of degree 18 may reach a few minutes, occasionally.

Together with the transfer target type $\tau(K)$ and the number $\varepsilon(K)$ of groups of type $(3,3,3)$, the structure of the 3 -class group $\mathrm{Cl}_{3}\left(\mathrm{~F}_{3}^{1}(K)\right)$ determines the principalization type $\varkappa(K)$ and the structure of the second 3-class group $G=\operatorname{Gal}\left(\mathrm{F}_{3}^{2}(K) \mid K\right)$ of the quadratic field $K$ in the following manner: up to separation of types E.6, E.14, resp. E.8, E.9, for $\operatorname{cc}(G)=2$ by means of Theorem 4.4 and up to separation of types F.7, F.11, F.12, F.13, resp. G.16, G.19,H.4, resp. d.19, d.23,d.25, for $\operatorname{cc}(G) \geq 3$ by means of Theorem 4.5 and [30, Thm. 5.1-5.3, pp. 491-494].

In our paper [30, we have indicated another computational technique, trying to avoid the use of the highly sophisticated system PARI/GP. The invariants of the second 3-class group $G=$ $\operatorname{Gal}\left(\mathrm{F}_{3}^{2}(K) \mid K\right)$, namely the class $\operatorname{cl}(G)=m-1$, coclass $\operatorname{cc}(G)=e-1$, and the order $|G|=3^{n}, n=$ $\operatorname{cl}(G)+\operatorname{cc}(G)$, are determined with the aid of 3 -class numbers $\mathrm{h}\left(L_{i}\right), 1 \leq i \leq 4$, of cubic fields, using [30, Thm. 5.1-5.3]. These class numbers can be computed by means of our own implementation of the classical Voronoi algorithms for calculating integral bases [43] and fundamental systems of units 44 for simply or totally real cubic fields and subsequent application of the analytic class number formula and Euler product method. The transfer kernel type $\varkappa$ of $G$ is assumed as an experimental input data in [30], produced by some unspecified principalization algorithm, for instance the classical algorithm in [40, 22, 11, 28. Consequently, the difficult determination of the defect $k$ with the aid of the Hilbert 3-class field $\mathrm{F}_{3}^{1}(K)$ of $K$ is circumvented, when the weak leaf conjecture 4.2 is assumed to hold. We have successfully applied this cumbersome classical procedure to the restricted range $-10^{5}<D<10^{6}$ of quadratic discriminants, for which we needed 7 years from 2003 to 2009 . However, the extension to $-10^{6}<D<10^{7}$ definitely requires the high 
speed performance of PARI/GP or MAGMA and the automatizability of our new principalization algorithm. Including the manual evaluation it was done within 5 months in 2010. CPU time added up to a total of a few weeks.

\section{NumericAl RESUlts ON SECOND 3-ClASS GROUPS OF 4596 QUADRATIC FIELDS}

By means of the principalization algorithm, implemented in the PARI/GP programs of $\S$ 罒 the principalization type $\varkappa(K)$ [31, Tbl. 6-7, pp. 492-493] and the structure of the second 3-class group $G=\mathrm{G}_{3}^{2}(K)=\operatorname{Gal}\left(\mathrm{F}_{3}^{2}(K) \mid K\right)$ [30 has been determined for the 2020 complex quadratic fields $K=\mathbb{Q}(\sqrt{D})$ with discriminant $-10^{6}<D<0$ and for the 2576 real quadratic fields $K=\mathbb{Q}(\sqrt{D})$ with discriminant $0<D<10^{7}$, having a 3-class group $\mathrm{Cl}_{3}(K)$ of type $(3,3)$. The results of these extensive computations reveal reliable statistical tendencies concerning the distribution of the groups $G$ on the sporadic part and various coclass trees of the coclass graphs $\mathcal{G}(3, r), 1 \leq r \leq 6$, [26, 25, 15] for a total of 4596 quadratic base fields.

Each of the following tables gives the characterizing transfer target type (TTT) $\tau$, as a novelty which was unknown up to now, and the minimal discriminant and absolute frequency of various transfer kernel types (TKTs) $\varkappa$. Here, 11 tables are arranged according to the sign of the discriminant $D$ and the graph theoretic location of the second 3-class group $G$, either on sporadic parts or on branches of coclass trees, which constitute the coclass graphs, thus providing necessary information for drawing graphical diagrams of coclass graphs in the subsequent paper [33. In contrast, the four Tables $2-5$ in our previous paper [30, $\S 6$, pp. 496-499] were arranged according to the number $\nu$ of total principalizations.

TABle 11. Principalization types with $G \in \mathcal{G}(3,1)$ for $D>0$

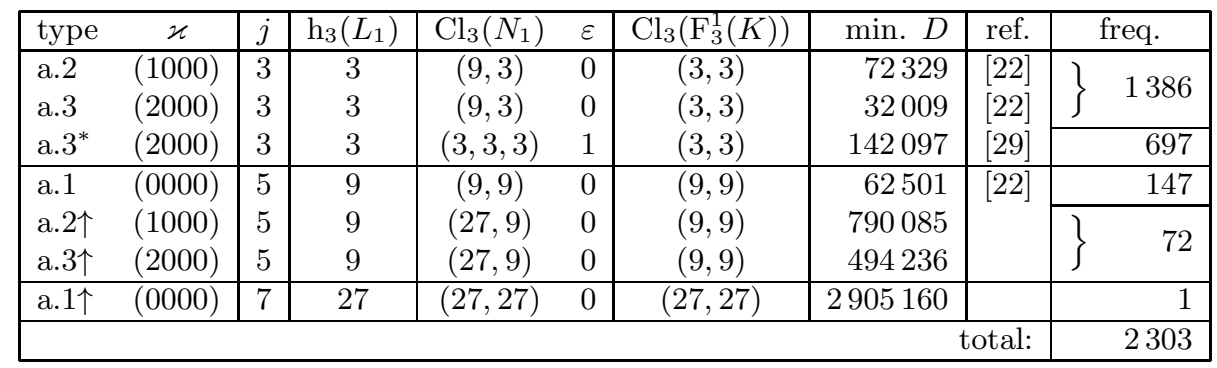

Table 11 characterizes the most frequent types $\varkappa$ of real quadratic fields with $G$ of coclass 1 by means of $\mathrm{h}_{3}\left(L_{1}\right)$ and $\mathrm{Cl}_{3}\left(N_{1}\right)$. For the other fields with $2 \leq i \leq 4$, we always have $\mathrm{h}_{3}\left(L_{i}\right)=3$ and $\mathrm{Cl}_{3}\left(N_{i}\right)$ of type $(3,3)$. The second 3-class group $G$ is a vertex of depth 1 on an odd branch $\mathcal{B}(j), j \in\{3,5,7\}$, of the unique coclass tree $\mathcal{T}\left(\mathrm{C}_{3} \times \mathrm{C}_{3}\right)$ of $\mathcal{G}(3,1)$ in Figure 1 Mainline groups of depth 0 do not occur and are probably impossible for quadratic fields, as stated in our Conjecture

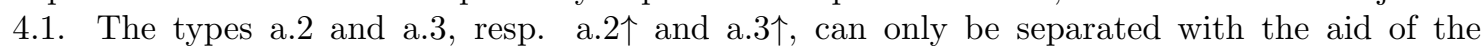
classical principalization algorithm 40,22 . Among the 2576 real quadratic fields $K=\mathbb{Q}(\sqrt{D})$ with discriminant $0<D<10^{7}$ and 3 -class group $\mathrm{Cl}_{3}(K)$ of type $(3,3)$, the dominating part of 2303 fields, that is $89.4 \%$, has a second 3 -class group $G$ of coclass $\operatorname{cc}(G)=1$. Branch $\mathcal{B}(3)$ is populated most densely by $\frac{697}{2303}=30.3 \%$ groups of type a. $3^{*}$ and $\frac{1386}{2303}=60.2 \%$ groups of types a.2 and a.3.

TABLE 12. Principalization types with $G \in \mathcal{T}(\langle 2187,64\rangle) \subset \mathcal{G}(3,3)$ for $D>0$

\begin{tabular}{|rc|cc|ccccc|c|r|r|}
\hline type & $\varkappa$ & $\mathrm{h}_{3}\left(L_{1}\right)$ & $\mathrm{h}_{3}\left(L_{2}\right)$ & $\mathrm{Cl}_{3}\left(N_{1}\right)$ & $\mathrm{Cl}_{3}\left(N_{2}\right)$ & $\mathrm{Cl}_{3}\left(N_{3}\right)$ & $\mathrm{Cl}_{3}\left(N_{4}\right)$ & $\varepsilon$ & $\mathrm{Cl}_{3}\left(\mathrm{~F}_{3}^{1}(K)\right)$ & min. $D$ & fr. \\
\hline b.10 & $(0043)$ & 9 & 9 & $(9,9)$ & $(9,9)$ & $(3,3,3)$ & $(3,3,3)$ & 2 & $(9,9,3,3)$ & 710652 & 8 \\
d.19 & $(4043)$ & 9 & 9 & $(27,9)$ & $(9,9)$ & $(3,3,3)$ & $(3,3,3)$ & 2 & $(9,9,3,3)$ & 2328721 & 1 \\
d.23 & $(1043)$ & 9 & 9 & $(27,9)$ & $(9,9)$ & $(3,3,3)$ & $(3,3,3)$ & 2 & $(9,9,3,3)$ & 1535117 & 1 \\
\hline \multicolumn{10}{|c}{ total: } & 10 \\
\hline
\end{tabular}


Table 12 characterizes the rare types $\varkappa$ of real quadratic fields with $G$ of coclass 3 by means of $\mathrm{h}_{3}\left(L_{i}\right), 1 \leq i \leq 2$, and $\mathrm{Cl}_{3}\left(N_{i}\right), 1 \leq i \leq 4$. For these cases we have $\mathrm{h}_{3}\left(L_{i}\right)=3$, for $3 \leq i \leq 4$, and the second 3 -class group $G$ is a vertex of depth 1 on the odd branch $\mathcal{B}(7)$ of the tree with $\operatorname{root}\langle 2187,64\rangle \simeq G_{0}^{5,7}(0,0,0,0)$ [36, p. $189 \mathrm{ff}$.] of $\mathcal{G}(3,3)$. The types d.19, d.23, d.25 [31, Thm. 3.4 , p. 491] can only be separated by the classical principalization algorithm [40, 22. Among the 2576 real quadratic fields under investigation, only a negligible part of 10 fields, that is $0.4 \%$, has a second 3-class group $G$ of coclass $\operatorname{cc}(G)=3$.

Whereas groups on $\mathcal{G}(3,1), \mathcal{G}(3,3)$, and generally groups with odd coclass, are impossible for complex quadratic fields [30, Thm. 4.2, p. 489], we now proceed to coclass graphs which are populated by second 3-class groups of quadratic fields with either sign of the discriminant.

TABle 13. Principalization types with sporadic $G \in \mathcal{G}(3,2)$ for $D>0$

\begin{tabular}{|c|c|c|c|c|c|c|c|c|c|c|c|}
\hline type & $\varkappa$ & $\mathrm{h}_{3}\left(L_{1}\right)$ & $\mathrm{h}_{3}\left(L_{2}\right)$ & $\mathrm{Cl}_{3}\left(N_{1}\right)$ & $\mathrm{Cl}_{3}\left(N_{2}\right)$ & $\mathrm{Cl}_{3}\left(N_{3}\right)$ & $\mathrm{Cl}_{3}\left(N_{4}\right)$ & $\varepsilon$ & $\mathrm{Cl}_{3}\left(\mathrm{~F}_{3}^{1}(K)\right)$ & min. $D$ & freq. \\
\hline G.19 & $(2143)$ & 3 & 3 & $(9,3)$ & $(9,3)$ & $(9,3)$ & $(9,3)$ & 0 & $(3,3,3,3)$ & 214712 & 11 \\
\hline D.10 & (2241) & 3 & 3 & $(9,3)$ & $(9,3)$ & $(3,3,3)$ & $(9,3)$ & 1 & $(3,3,3)$ & 422573 & 93 \\
\hline D. 5 & $(4224)$ & 3 & 3 & $(3,3,3)$ & $(9,3)$ & $(3,3,3)$ & $(9,3)$ & 2 & $(3,3,3)$ & 631769 & 47 \\
\hline \multirow[t]{2}{*}{ H.4 } & (4443) & 3 & 3 & $(3,3,3)$ & $(3,3,3)$ & $(9,3)$ & $(3,3,3)$ & 3 & $(9,3,3)$ & 957013 & 27 \\
\hline & & & & & & & & & & total: & 178 \\
\hline
\end{tabular}

The second 3 -class groups $G$ for types $\varkappa$ in Table 13 are sporadic vertices on $\mathcal{G}(3,2)$ in Figure 2. They are determined uniquely by $\varepsilon$ (Cor. 4.2.2). Among the 263 groups $G$ of even coclass for real quadratic fields, a contribution of 178 groups, that is $67.7 \%$, is sporadic of coclass $\operatorname{cc}(G)=2$. This is the adequate kind of relative frequencies for comparison with complex quadratic fields.

TABLE 14. Principalization types with sporadic $G \in \mathcal{G}(3,2)$ for $D<0$

\begin{tabular}{|c|c|c|c|c|c|c|c|c|c|c|c|}
\hline type & $x$ & $\mathrm{~h}\left(L_{1}\right)$ & $\mathrm{h}\left(L_{2}\right)$ & $\mathrm{Cl}_{3}\left(N_{1}\right)$ & $\mathrm{Cl}_{3}\left(N_{2}\right)$ & $\mathrm{Cl}_{3}\left(N_{3}\right)$ & $\mathrm{Cl}_{3}\left(N_{4}\right)$ & $\varepsilon$ & $\mathrm{Cl}_{3}\left(\mathrm{~F}_{3}^{1}(K)\right)$ & $\min .|D|$ & freq. \\
\hline G.19 & $(2143)$ & 3 & 3 & $(9,3)$ & $(9,3)$ & $(9,3)$ & $(9,3)$ & $\overline{0}$ & $(3,3,3,3)$ & 12067 & 94 \\
\hline D.10 & (2241) & 3 & 3 & $(9,3)$ & $(9,3)$ & $(3,3,3)$ & $(9,3)$ & 1 & $(3,3,3)$ & 4027 & 667 \\
\hline D.5 & $(4224)$ & 3 & 3 & $(3,3,3)$ & $(9,3)$ & $(3,3,3)$ & $(9,3)$ & 2 & $(3,3,3)$ & 12131 & 269 \\
\hline & (4443) & 3 & 3 & $(3,3,3)$ & $(3,3,3)$ & $(9,3)$ & $(3,3,3)$ & 3 & $(9,3,3)$ & 3896 & 297 \\
\hline H.4 & & & & & & & & & & total: & 1327 \\
\hline
\end{tabular}

Table 14 is the complex counterpart of Table13. Among the 2020 complex quadratic fields $K=$ $\mathbb{Q}(\sqrt{D})$ with discriminant $-10^{6}<D<0$ and 3-class group $\mathrm{Cl}_{3}(K)$ of type $(3,3)$ a considerable part of 1327 fields, that is $65.7 \%$, has a sporadic second 3-class group $G$ of $\operatorname{coclass} \operatorname{cc}(G)=2$. Type D.10 with a relative frequency of $\frac{667}{2020}=33.0 \%$ is the absolute high-champ for complex quadratic fields.

TABLE 15. Principalization types with $G \in \mathcal{T}(\langle 243,6\rangle) \subset \mathcal{G}(3,2)$ for $D>0$

\begin{tabular}{|c|c|c|c|c|c|c|c|c|c|c|c|c|}
\hline type & $\varkappa$ & $j$ & $\mathrm{~h}_{3}\left(L_{1}\right)$ & $\mathrm{h}_{3}\left(L_{2}\right)$ & $\overline{\mathrm{Cl}_{3}\left(N_{1}\right)}$ & $\overline{\mathrm{Cl}_{3}\left(N_{2}\right)}$ & $\mathrm{Cl}_{3}\left(N_{3}\right)$ & $\mathrm{Cl}_{3}\left(N_{4}\right)$ & $\varepsilon$ & $\mathrm{Cl}_{3}\left(\mathrm{~F}_{3}^{1}(K)\right)$ & $\min . D$ & freq. \\
\hline $\begin{array}{ll}\text { c. } 18 \\
\end{array}$ & (0313) & 6 & 9 & 3 & $(9,9)$ & $(9,3)$ & $(3,3,3)$ & $(9,3)$ & 1 & $(9,3,3)$ & 534824 & 29 \\
\hline E. 6 & (1313) & 6 & 9 & 3 & $(27,9)$ & $(9,3)$ & $(3,3,3)$ & $(9,3)$ & 1 & $(9,9,3)$ & 5264069 & 7 \\
\hline E.14 & (2313) & 6 & 9 & 3 & $(27,9)$ & $(9,3)$ & $(3,3,3)$ & $(9,3)$ & 1 & $(9,9,3)$ & 3918837 & 7 \\
\hline $\mathrm{H} .4 \uparrow$ & (3313) & 6 & 9 & 3 & $(27,9)$ & $(9,3)$ & $(3,3,3)$ & $(9,3)$ & 1 & $(27,9,3)$ & 1162949 & 3 \\
\hline & & & & & & & & & & & total: & 39 \\
\hline
\end{tabular}

The second 3-class groups $G$ for types $\varkappa$ in Table 15 are vertices on the coclass tree with root $\langle 243,6\rangle \simeq G_{0}^{4,5}(0,-1,0,1)$ of $\mathcal{G}(3,2)$. $G$ is a vertex of depth 0 for type c.18, of depth 1 for types E.6, E.14, and of depth 2 for type H.4, on the even branch $\mathcal{B}(6)$, [3, Tbl. 2, p. 266], [4, Fig. 4.8, 
p. 76], [36, p. 189 ff.]. The types E.6, E.14 can only be separated by the classical principalization algorithm [40, 22. Type H.4 can be identified either by the first Hilbert 3-class field or by the classical principalization algorithm. Among the 263 groups of even coclass for real quadratic fields, a fraction of 39 groups, that is $14.8 \%$, populates this tree.

TABLE 16. Principalization types with $G \in \mathcal{T}(\langle 243,6\rangle) \subset \mathcal{G}(3,2)$ for $D<0$

\begin{tabular}{|c|c|c|c|c|c|c|c|c|c|c|c|c|}
\hline type & $\varkappa$ & $j$ & $\mathrm{~h}\left(L_{1}\right)$ & $\mathrm{h}\left(L_{2}\right)$ & $\mathrm{Cl}_{3}\left(N_{1}\right)$ & $\mathrm{Cl}_{3}\left(N_{2}\right)$ & $\mathrm{Cl}_{3}\left(N_{3}\right)$ & $\mathrm{Cl}_{3}\left(N_{4}\right)$ & $\varepsilon$ & $\mathrm{Cl}_{3}\left(\mathrm{~F}_{3}^{1}(K)\right)$ & $\min .|D|$ & \multirow{3}{*}{$\begin{array}{c}\text { freq. } \\
\} 186\end{array}$} \\
\hline E.6 & (1313) & 6 & 9 & 3 & $(27,9)$ & $(9,3)$ & $(3,3,3)$ & $(9,3)$ & 1 & $(9,9,3)$ & 15544 & \\
\hline E.14 & (2313) & 6 & 9 & 3 & $(27,9)$ & $(9,3)$ & $(3,3,3)$ & $(9,3)$ & 1 & $(9,9,3)$ & 16627 & \\
\hline H. $4 \uparrow$ & (3313) & 6 & 9 & 3 & $(27,9)$ & $(9,3)$ & $(3,3,3)$ & $(9,3)$ & 1 & $(27,9,3)$ & 21668 & 63 \\
\hline$\overline{\mathrm{E} .6 \uparrow}$ & $(1313)$ & $\overline{8}$ & 27 & $\overline{3}$ & $(81,27)$ & $\overline{(9,3)}$ & $(3,3,3)$ & $\overline{(9,3)}$ & 1 & $(27,27,3)$ & 268040 & \multirow{2}{*}{\}$\quad 15$} \\
\hline E. $14 \uparrow$ & (2313) & 8 & 27 & 3 & $(81,27)$ & $(9,3)$ & $(3,3,3)$ & $(9,3)$ & 1 & $(27,27,3)$ & 262744 & \\
\hline $\mathrm{H} .4 \uparrow^{2}$ & (3313) & 8 & 27 & 3 & $(81,27)$ & $(9,3)$ & $(3,3,3)$ & $(9,3)$ & 1 & $(81,27,3)$ & 446788 & 6 \\
\hline & & & & & & & & & & & total: & 270 \\
\hline
\end{tabular}

Again, we continue opposing the complex analog to Table 15 in Table 16, Among the 2020 complex quadratic fields under investigation, a considerable fraction of 270 fields, that is $13.4 \%$, has a second 3-class group $G$ on the coclass tree $\mathcal{T}(\langle 243,6\rangle)$ of $\mathcal{G}(3,2)$. However, the groups now populate two even branches $\mathcal{B}(j), j \in\{6,8\}$, of this tree.

TABle 17. Principalization types with $G \in \mathcal{T}(\langle 243,8\rangle) \subset \mathcal{G}(3,2)$ for $D>0$

\begin{tabular}{|c|c|c|c|c|c|c|c|c|c|c|c|c|}
\hline type & $\varkappa$ & $j$ & $\mathrm{~h}_{3}\left(L_{1}\right)$ & $\mathrm{h}_{3}\left(L_{2}\right)$ & $\mathrm{Cl}_{3}\left(N_{1}\right)$ & $\mathrm{Cl}_{3}\left(N_{2}\right)$ & $\mathrm{Cl}_{3}\left(N_{3}\right)$ & $\mathrm{Cl}_{3}\left(N_{4}\right)$ & $\varepsilon$ & $\mathrm{Cl}_{3}\left(\mathrm{~F}_{3}^{1}(K)\right)$ & min. $D$ & freq. \\
\hline $\begin{array}{ll}\text { c. } 21 \\
\end{array}$ & $(0231)$ & 6 & 9 & 3 & $(9,9)$ & $(9,3)$ & $(9,3)$ & $(9,3)$ & 0 & \multirow{2}{*}{$\begin{array}{l}(9,3,3) \\
(9,9,3)\end{array}$} & \multirow{2}{*}{$\begin{array}{r}540365 \\
6098360\end{array}$} & 25 \\
\hline E. 8 & (1231) & 6 & 9 & 3 & $(27,9)$ & $(9,3)$ & $(9,3)$ & $(9,3)$ & 0 & & & \\
\hline E.9 & (2231) & 6 & 9 & 3 & $(27,9)$ & $(9,3)$ & $(9,3)$ & $(9,3)$ & 0 & $(9,9,3)$ & 342664 & \} \\
\hline G.16 & (4231) & 6 & 9 & 3 & $(27,9)$ & $(9,3)$ & $(9,3)$ & $(9,3)$ & 0 & $(27,9,3)$ & 8711453 & 2 \\
\hline c. $21 \uparrow$ & $(0231)$ & 8 & 27 & 3 & $(27,27)$ & $(9,3)$ & $(9,3)$ & $(9,3)$ & 0 & $(27,9,3)$ & 1001957 & 2 \\
\hline & & & & & & & & & & & total: & $\overline{43}$ \\
\hline
\end{tabular}

The second 3 -class groups $G$ for types $\varkappa$ in Table 17 are vertices on the coclass tree with root $\langle 243,8\rangle \simeq G_{0}^{4,5}(0,0,0,1)$ of $\mathcal{G}(3,2)$. $G$ is a vertex of depth 0 for type c.21, of depth 1 for types E.8, E.9, and of depth 2 for type G.16, on the even branches $\mathcal{B}(j), j \in\{6,8\}$, [3, Tbl. 2, p. 266], [4, Fig. 4.8, p. 76], [36, p. 189 ff.]. The types E.8, E.9 can only be separated by the classical principalization algorithm [40, 22. Type G.16 can be identified either by the first Hilbert 3 -class field or by the classical principalization algorithm. Among the 263 groups of even coclass, a fraction of 43 groups, that is $16.3 \%$, populates this tree.

TABle 18. Principalization types with $G \in \mathcal{T}(\langle 243,8\rangle) \subset \mathcal{G}(3,2)$ for $D<0$

\begin{tabular}{|c|c|c|c|c|c|c|c|c|c|c|c|c|}
\hline type & $\varkappa$ & $j$ & $\mathrm{~h}\left(L_{1}\right)$ & $\mathrm{h}\left(L_{2}\right)$ & $\mathrm{Cl}_{3}\left(N_{1}\right)$ & $\mathrm{Cl}_{3}\left(N_{2}\right)$ & $\mathrm{Cl}_{3}\left(N_{3}\right)$ & $\mathrm{Cl}_{3}\left(N_{4}\right)$ & $\varepsilon$ & $\mathrm{Cl}_{3}\left(\mathrm{~F}_{3}^{1}(K)\right)$ & $\min .|D|$ & freq. \\
\hline E. 8 & $\overline{(1231)}$ & 6 & 9 & 3 & $(27,9)$ & $(9,3)$ & $(9,3)$ & $(9,3)$ & $\overline{0}$ & $(9,9,3)$ & 34867 & \multirow{3}{*}{197} \\
\hline E.9 & (2231) & 6 & 9 & 3 & $(27,9)$ & $(9,3)$ & $(9,3)$ & $(9,3)$ & 0 & $(9,9,3)$ & 9748 & \\
\hline G.16 & (4231) & 6 & 9 & 3 & $(27,9)$ & $(9,3)$ & $(9,3)$ & $(9,3)$ & 0 & $(27,9,3)$ & 17131 & \\
\hline E. $8 \uparrow$ & (1231) & 8 & 27 & 3 & $(81,27)$ & $(9,3)$ & $(9,3)$ & $(9,3)$ & $\overline{0}$ & $(27,27,3)$ & 370740 & \multirow{2}{*}{\}$\quad 13$} \\
\hline E. $9 \uparrow$ & (2231) & 8 & 27 & 3 & $(81,27)$ & $(9,3)$ & $(9,3)$ & $(9,3)$ & 0 & $(27,27,3)$ & 297079 & \\
\hline G.16个 & (4231) & 8 & 27 & 3 & $(81,27)$ & $(9,3)$ & $(9,3)$ & $(9,3)$ & 0 & $(81,27,3)$ & 819743 & 2 \\
\hline & & & & & & & & & & & total: & 291 \\
\hline
\end{tabular}

Table 18 is the complex analog of Table 17. Among the 2020 complex quadratic fields under investigation, a considerable fraction of 291 fields, that is $14.4 \%$, has a second 3 -class group $G$ on the coclass tree $\mathcal{T}(\langle 243,8\rangle)$ of $\mathcal{G}(3,2)$. 
TABLE 19. Principalization types with $G \in \mathcal{G}(3,4)$ for $D>0$

\begin{tabular}{|lc|cc|ccccc|c|r|r|}
\hline type & $\varkappa$ & $\mathrm{h}_{3}\left(L_{1}\right)$ & $\mathrm{h}_{3}\left(L_{2}\right)$ & $\mathrm{Cl}_{3}\left(N_{1}\right)$ & $\mathrm{Cl}_{3}\left(N_{2}\right)$ & $\mathrm{Cl}_{3}\left(N_{3}\right)$ & $\mathrm{Cl}_{3}\left(N_{4}\right)$ & $\varepsilon$ & $\mathrm{Cl}_{3}\left(\mathrm{~F}_{3}^{1}(K)\right)$ & $\min . D$ & fr. \\
\hline $\mathrm{F} .13$ & $(3143)$ & 9 & 9 & $(27,9)$ & $(27,9)$ & $(3,3,3)$ & $(3,3,3)$ & 2 & $(9,9,9,3)$ & 8321505 & 1 \\
$\mathrm{~F} .13 \uparrow$ & $(3143)$ & 27 & 9 & $(81,27)$ & $(27,9)$ & $(3,3,3)$ & $(3,3,3)$ & 2 & $(27,27,9,3)$ & 8127208 & 1 \\
\hline d.25* & $(0143)$ & 27 & 9 & $(27,27)$ & $(27,9)$ & $(3,3,3)$ & $(3,3,3)$ & 2 & $(27,9,9,3)$ & 8491713 & 1 \\
\hline \multicolumn{10}{|c}{ total: } & 3 \\
\hline
\end{tabular}

The extremely rare second 3-class groups $G$ for types $\varkappa$ in Table 19 are vertices on the coclass graph $\mathcal{G}(3,4)$. The group $G$ is either a sporadic vertex outside of coclass trees for type F.13, or a vertex on the even branch $\mathcal{B}(10)$ of one of the five metabelian coclass trees of $\mathcal{G}(3,4)$, a vertex of depth 1 for type F.13个, and a main line vertex of depth 0 for type d.25* [36, p. 189 ff.], [31, Thm. 3.4, p. 491]. The types F.13, F.13个, and d.25* must be identified by the classical principalization algorithm [40, 22. Among the 263 groups of even coclass for real quadratic fields, a very exotic fraction of only 3 groups, that is $1.1 \%$, populates $\mathcal{G}(3,4)$. The population sets in with remarkable delay at $D>8 \cdot 10^{6}$.

TABle 20. Principalization types with $G \in \mathcal{G}(3,4)$ for $D<0$

\begin{tabular}{|c|c|c|c|c|c|c|c|c|c|c|c|}
\hline type & $\varkappa$ & $\overline{\mathrm{h}\left(L_{1}\right)}$ & $\overline{\mathrm{h}\left(L_{2}\right)}$ & $\mathrm{Cl}_{3}\left(N_{1}\right)$ & $\overline{\mathrm{Cl}_{3}\left(N_{2}\right)}$ & $\overline{\mathrm{Cl}_{3}\left(N_{3}\right)}$ & $\mathrm{Cl}_{3}\left(N_{4}\right)$ & $\varepsilon$ & $\mathrm{Cl}_{3}\left(\mathrm{~F}_{3}^{1}(K)\right)$ & $\min .|D|$ & freq. \\
\hline F.7 & $(3443)$ & 9 & 9 & $(27,9)$ & $(27,9)$ & $(3,3,3)$ & $(3,3,3)$ & 2 & $(9,9,9,3)$ & 124363 & \multirow{4}{*}{78} \\
\hline F.11 & (1143) & 9 & 9 & $(27,9)$ & $(27,9)$ & $(3,3,3)$ & $(3,3,3)$ & 2 & $(9,9,9,3)$ & 27156 & \\
\hline F.12 & (1343) & 9 & 9 & $(27,9)$ & $(27,9)$ & $(3,3,3)$ & $(3,3,3)$ & 2 & $(9,9,9,3)$ & 31908 & \\
\hline F.13 & (3143) & 9 & 9 & $(27,9)$ & $(27,9)$ & $(3,3,3)$ & $(3,3,3)$ & 2 & $(9,9,9,3)$ & 67480 & \\
\hline F. $7 \uparrow$ & (3443) & 27 & 9 & $(81,27)$ & $(27,9)$ & $(3,3,3)$ & $(3,3,3)$ & 2 & $(27,27,9,3)$ & 469816 & \multirow{4}{*}{14} \\
\hline F.11个 & (1143) & 27 & 9 & $(81,27)$ & $(27,9)$ & $(3,3,3)$ & $(3,3,3)$ & 2 & $(27,27,9,3)$ & 469787 & \\
\hline F. $12 \uparrow$ & (1343) & 27 & 9 & $(81,27)$ & $(27,9)$ & $(3,3,3)$ & $(3,3,3)$ & 2 & $(27,27,9,3)$ & 249371 & \\
\hline F. $13 \uparrow$ & (3143) & 27 & 9 & $(81,27)$ & $(27,9)$ & $(3,3,3)$ & $(3,3,3)$ & 2 & $(27,27,9,3)$ & 159208 & \\
\hline G.16r & $(1243)$ & 9 & 9 & $(27,9)$ & $(27,9)$ & $(3,3,3)$ & $(3,3,3)$ & 2 & $(27,9,9,3)$ & 290703 & \multirow{3}{*}{19} \\
\hline G.19r & (2143) & 9 & 9 & $(27,9)$ & $(27,9)$ & $(3,3,3)$ & $(3,3,3)$ & 2 & $(27,9,9,3)$ & 96827 & \\
\hline H. $4 \mathrm{r}$ & (3343) & 9 & 9 & $(27,9)$ & $(27,9)$ & $(3,3,3)$ & $(3,3,3)$ & 2 & $(27,9,9,3)$ & 256935 & \\
\hline G.16i & (1243) & 9 & 9 & $(27,9)$ & $(27,9)$ & $(3,3,3)$ & $(3,3,3)$ & 2 & $(9,9,9,9)$ & 135059 & \multirow{3}{*}{15} \\
\hline G.19i & $(2143)$ & 9 & 9 & $(27,9)$ & $(27,9)$ & $(3,3,3)$ & $(3,3,3)$ & 2 & $(9,9,9,9)$ & 199735 & \\
\hline $\mathrm{H} .4 \mathrm{i}$ & $(3343)$ & 9 & 9 & $(27,9)$ & $(27,9)$ & $(3,3,3)$ & $(3,3,3)$ & 2 & $(9,9,9,9)$ & 186483 & \\
\hline$\overline{\mathrm{G} .19 \mathrm{r} \uparrow}$ & $(2143)$ & 27 & 9 & $(81,27)$ & $(27,9)$ & $(3,3,3)$ & $(3,3,3)$ & 2 & $(81,27,9,3)$ & 509160 & \multirow{2}{*}{$\begin{array}{l}2 \\
3\end{array}$} \\
\hline $\mathrm{H} .4 \mathrm{r} \uparrow$ & (3343) & 27 & 9 & $(81,27)$ & $(27,9)$ & $(3,3,3)$ & $(3,3,3)$ & 2 & $(81,27,9,3)$ & 678804 & \\
\hline & & & & & & & & & & total: & 131 \\
\hline
\end{tabular}

In contrast, Table 20 shows that the coclass graph $G \in \mathcal{G}(3,4)$ accommodates the second 3class groups $G$ of quite a notable portion of 131 , that is $6.5 \%$, among the 2020 complex quadratic fields under investigation. The group $G$ is either a sporadic vertex outside of coclass trees, namely an isolated metabelian top vertex of $\mathcal{G}(3,4)$ for the types F.7, F.11, F.12, F.13 and a terminal metabelian vertex of depth 1 on a finite tree for the types G.16r, G.19r, H.4r, G.16i, G.19i, H.4i, or a vertex on the even branch $\mathcal{B}(10)$ of one of the five metabelian coclass trees of $\mathcal{G}(3,4)$, namely

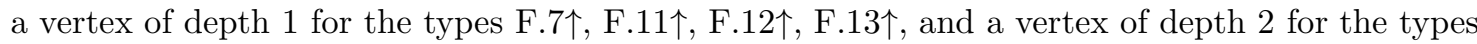
G.19r $\uparrow$, H.4r $\uparrow$. We point out that the classical principalization algorithm [40, 22, which must be used to separate the types F.7, F.11, F.12, F.13, resp. G.16, G.19, H.4, is not able to reveal that the types G.16, G.19, and H.4 with odd index of nilpotency $m=7$ and order $3^{n}, n=10=2 m-4$, can appear in a regular variant $(\mathrm{r})$ with $\mathrm{Cl}_{3}\left(\mathrm{~F}_{3}^{1}(K)\right)$ of type $(27,9,9,3)$ and an irregular variant (i) with $\mathrm{Cl}_{3}\left(\mathrm{~F}_{3}^{1}(K)\right)$ of type $(9,9,9,9)$ [36, Satz 4.2.4, p. 131].

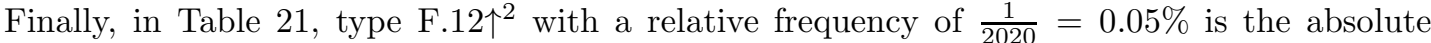
low-champ for complex quadratic fields. The corresponding second 3-class group $G$ is of the biggest order $3^{13}$, known until now. It is the unique sporadic vertex outside of coclass trees 
TABLE 21. Principalization type with $G \in \mathcal{G}(3,6)$ for $D<0$

\begin{tabular}{|c|c|c|c|c|c|c|c|c|c|c|c|}
\hline type & $\varkappa$ & $\mathrm{h}\left(L_{1}\right)$ & $\mathrm{h}\left(L_{2}\right)$ & $\mathrm{Cl}_{3}\left(N_{1}\right)$ & $\mathrm{Cl}_{3}\left(N_{2}\right)$ & $\mathrm{Cl}_{3}\left(N_{3}\right)$ & $\mathrm{Cl}_{3}\left(N_{4}\right)$ & $\varepsilon$ & $\mathrm{Cl}_{3}\left(\mathrm{~F}_{3}^{1}(K)\right)$ & $\min .|D|$ & freq. \\
\hline$\overline{\mathrm{F} .12 \uparrow^{2}}$ & $(1343)$ & 27 & 27 & $(81,27)$ & $(81,27)$ & $(3,3,3)$ & $(3,3,3)$ & 2 & $(27,27,27,9)$ & 423640 & 1 \\
\hline
\end{tabular}

which appeared on the coclass graph $\mathcal{G}(3,6)$. Type F.12 $\uparrow^{2}$ must be identified by the classical principalization algorithm [40, 22].

Remark 6.1. Based on the statistical evaluation of all numerical results, we are able to conclude that the new principalization algorithm is significantly more efficient for real quadratic fields. The time consuming third step, $\S 5.3$, of the algorithm can be avoided for $\frac{2548}{2576}=98.9 \%$ of the real quadratic fields (Tables 11, 12, 13 entirely and additionally the 29 cases of type c.18 in Table 15, the 27 cases of type c.21 in Table 17, and the single case of type d.25* in Table 19), but only for $\frac{1327}{2020}=65.7 \%$ of the complex quadratic fields (Table 14).

\section{AcKnowledgements}

The author expresses his gratitude to Mike F. Newman, ANU, Canberra, for precious aid in unifying the various approaches [3, 4, 5, 6, 9, 13, 20, 21, 23, 36, 41] to the classification of finite metabelian 3-groups and in discovering the last statement of Corollary 4.3.2. Further he would like to thank Karim Belabas, Univ. Bordeaux, and Claus Fieker, Univ. Kaiserslautern, for illuminating discussions concerning the computation of arithmetical invariants of Hilbert 3-class fields over quadratic fields [7, 17, using the computer algebra systems PARI/GP and MAGMA. Finally, the author is endebted to the anonymous referee for valuable suggestions to improve the exposition.

\section{REFERENCES}

[1] E. Artin, Beweis des allgemeinen Reziprozitätsgesetzes, Abh. Math. Sem. Univ. Hamburg 5 (1927), 353-363.

[2] E. Artin, Idealklassen in Oberkörpern und allgemeines Reziprozitätsgesetz, Abh. Math. Sem. Univ. Hamburg 7 (1929), 46-51.

[3] J. Ascione, G. Havas, and C. R. Leedham-Green, A computer aided classification of certain groups of prime power order, Bull. Austral. Math. Soc. 17 (1977), 257-274, Corrigendum 317-319, Microfiche Supplement p.320.

[4] J. Ascione, On 3-groups of second maximal class (Ph.D. Thesis, Australian National University, Canberra, 1979).

[5] J. Ascione, On 3-groups of second maximal class, Bull. Austral. Math. Soc. 21 (1980), 473-474.

[6] G. Bagnera, La composizione dei gruppi finiti il cui grado è la quinta potenza di un numero primo, Ann. di Mat. (Ser. 3) 1 (1898), 137-228.

[7] K. Belabas, Topics in computational algebraic number theory, J. Théor. Nombres Bordeaux 16 (2004), 19-63.

[8] H. U. Besche, B. Eick, and E. A. O'Brien, The SmallGroups Library - a Library of Groups of Small Order, 2005, an accepted and refereed GAP 4 package, available also in MAGMA.

[9] N. Blackburn, On a special class of p-groups, Acta Math. 100 (1958), 45-92.

[10] N. Blackburn, On prime-power groups in which the derived group has two generators, Proc. Camb. Phil. Soc. 53 (1957), 19-27.

[11] J. R. Brink, The class field tower for imaginary quadratic number fields of type $(3,3)$ (Dissertation, Ohio State Univ., 1984).

[12] H. Dietrich, B. Eick, and D. Feichtenschlager, Investigating $p$-groups by coclass with GAP, Computational group theory and the theory of groups, 45-61 (Contemp. Math. 470, AMS, Providence, RI, 2008).

[13] T. E. Easterfield, A classification of groups of order $p^{6}$ (Ph. D. Thesis, Univ. of Cambridge, 1940).

[14] B. Eick and D. Feichtenschlager, Infinite sequences of p-groups with fixed coclass (arXiv: 1006.0961 v1 [math.GR], 4 Jun 2010).

[15] B. Eick and C. Leedham-Green, On the classification of prime-power groups by coclass, Bull. London Math. Soc. 40 (2008), 274-288.

[16] B. Eick, C.R. Leedham-Green, M.F. Newman, and E.A. O'Brien, On the classification of groups of primepower order by coclass: The 3-groups of coclass 2 (preprint, 2011).

[17] C. Fieker, Computing class fields via the Artin map, Math. Comp. 70 (2001), no.235, 1293-1303.

[18] G. W. Fung and H. C. Williams, On the computation of a table of complex cubic fields with discriminant $D>-10^{6}$, Math. Comp. 55 (1990), nr. 191, 313-325. 
[19] Ph. Furtwängler, Beweis des Hauptidealsatzes für die Klassenkörper algebraischer Zahlkörper, Abh. Math. Sem. Univ. Hamburg 7 (1929), 14-36.

[20] The GAP Group, GAP - Groups, Algorithms, and Programming — a System for Computational Discrete Algebra, Version 4.4.12, Aachen, Braunschweig, Fort Collins, St. Andrews, 2008, (\protect\vrule widthOpt \protect \href\{http://www.gap-system.org\}\{http://www.gap-system.org\}).

[21] P. Hall, The classification of prime-power groups, J. Reine Angew. Math. 182 (1940), 130-141.

[22] F.-P. Heider und B. Schmithals, Zur Kapitulation der Idealklassen in unverzweigten primzyklischen Erweiterungen, J. Reine Angew. Math. 336 (1982), 1-25.

[23] R. James, The groups of order $p^{6}$ ( $p$ an odd prime), Math. Comp. 34 (1980), nr. 150, 613-637.

[24] H. Kisilevsky, Some results related to Hilbert's theorem 94, J. Number Theory 2 (1970), 199-206.

[25] C. R. Leedham-Green and S. McKay, The structure of groups of prime power order, London Math. Soc. Monographs, New Series, 27, Oxford Univ. Press, 2002.

[26] C. R. Leedham-Green and M. F. Newman, Space groups and groups of prime power order I, Arch. Math. 35 (1980), 193-203.

[27] P. Llorente and J. Quer, On totally real cubic fields with discriminant $D<10^{7}$, Math. Comp. 50 (1988), nr. 182, 581-594.

[28] D. C. Mayer, Principalization in complex $S_{3}$-fields, Congressus Numerantium 80 (1991), $73-87$ (Proceedings of the Twentieth Manitoba Conference on Numerical Mathematics and Computing, Winnipeg, Manitoba, Canada, 1990).

[29] D. C. Mayer, List of discriminants $d_{L}<200000$ of totally real cubic fields L, arranged according to their multiplicities $m$ and conductors $f$ (Computer Centre, Department of Computer Science, University of Manitoba, Winnipeg, Canada, 1991).

[30] D. C. Mayer, The second p-class group of a number field, Int. J. Number Theory 8 (2012), no. 2, 471-505, DOI 10.1142/S179304211250025X.

[31] D. C. Mayer, Transfers of metabelian p-groups, Monatsh. Math. 166 (2012), no. 3-4, 467-495, DOI 10.1007/s00605-010-0277-x.

[32] D. C. Mayer, Multiplicities of dihedral discriminants, Math. Comp. 58 (1992), no.198, 831-847 and S55-S58.

[33] D. C. Mayer, The distribution of second p-class groups on coclass graphs, to appear in J. Théor. Nombres Bordeaux (2013).

[34] D. C. Mayer, Metabelian 3-groups with abelianization of type (9,3), (Preprint 2011).

[35] R. J. Miech, Metabelian p-groups of maximal class, Trans. Amer. Math. Soc. 152 (1970), 331-373.

[36] B. Nebelung, Klassifikation metabelscher 3-Gruppen mit Faktorkommutatorgruppe vom Typ (3,3) und Anwendung auf das Kapitulationsproblem (Inauguraldissertation, Band 1, Universität zu Köln, 1989).

[37] B. Nebelung, Anhang zu Klassifikation metabelscher 3-Gruppen mit Faktorkommutatorgruppe vom Typ $(3,3)$ und Anwendung auf das Kapitulationsproblem (Inauguraldissertation, Band 2, Universität zu Köln, 1989).

[38] The PARI Group, PARI/GP, Version 2.3.4, Bordeaux, 2008, (\protect \vrule width0pt \protect \href $\{$ http://pari.math.u-bordeaux.

[39] A. Scholz, Idealklassen und Einheiten in kubischen Körpern, Monatsh. Math. Phys. 40 (1933), 211-222.

[40] A. Scholz und O. Taussky, Die Hauptideale der kubischen Klassenkörper imaginär quadratischer Zahlkörper: ihre rechnerische Bestimmung und ihr Einfluß auf den Klassenkörperturm, J. Reine Angew. Math. 171 (1934), $19-41$.

[41] O. Schreier, Über die Erweiterung von Gruppen II, Abh. Math. Sem. Univ. Hamburg 4 (1926), 321-346.

[42] O. Taussky, A remark concerning Hilbert's Theorem 94, J. Reine Angew. Math. 239/240 (1970), 435-438.

[43] Г. Ф. Вороной, О целых алгебраических числах зависящих от корня уравнения третьей степени, Санктпетербург, Master's Thesis (Russian), 1894. Engl. transl. of title: Concerning algebraic integers derivable from a root of an equation of the third degree.

[44] Г. Ф. Вороной, Об одном обобщении алгорифма непрерывных дробей, Варшава, Doctoral Dissertation (Russian), 1896. Engl. transl. of title: On a generalization of the algorithm of continued fractions. Summary (by Wassilieff): Jahrb. Fortschr. Math. 27 (1896), 170-174.

\section{ApPENDix}

For the convenience of the reader, we literally cite some original results of Blackburn [9], and as a service to the mathematical community, we give a succinct survey of Nebelung's thesis [36, 37. Both of these works are used essentially in our article.

8.1. A theorem by Blackburn. Let $p$ be an arbitrary prime and observe that Blackburn denotes our two-step centralizer $\chi_{2}(G)$ of a $p$-group $G$ (see $\S$ 3.1) by $\gamma_{1}(G)$, whereas his usage of $\gamma_{i}(G)$ for $i \geq 2$ coincides with ours, denoting the members of the lower central series. The following theorem shows that all subgroups $\gamma_{i}(G), 1 \leq i \leq m-p+1$, of a $p$-group $G$ of $\operatorname{coclass} \operatorname{cc}(G)=1$ and class $\operatorname{cl}(G)=m-1>p$ are regular and have the same invariants as the nearly homocyclic 
abelian $p$-group $\mathrm{A}(p, m-i)$ of type $(\overbrace{p^{q_{i}+1}, \ldots, p^{q_{i}+1}}^{r_{i} \text { times }}, \overbrace{p^{q_{i}}, \ldots, p^{q_{i}}}^{p-1-r_{i} \text { times }})$, where $m-i=q_{i}(p-1)+r_{i}$ by Euclidean division with quotient $q_{i}>0$ and remainder $0 \leq r_{i}<p-1$. In particular, if $G$ is metabelian, then $\gamma_{i}(G) \simeq \mathrm{A}(p, m-i)$, for $2 \leq i \leq m-p+1$, and if $G$ is metabelian with defect $k(G)=0$, then also $\gamma_{1}(G) \simeq \mathrm{A}(p, m-1)$.

Theorem 8.1. (see [9, Thm. 3.4, p. 68]) If $G$ is a group of order $p^{m}$ and class $m-1$, where $m>3$, then $\gamma_{1}(G)$ is a regular $p$-group. If $m>p+1$, and for each $i=1,2, \ldots, m-p+1$, we write $m-i=(p-1) q_{i}+r_{i}\left(0 \leq r_{i}<p-1\right)$, then $\gamma_{i}(G)$ has $r_{i}$ invariants equal to $q_{i}+1$ and $p-r_{i}-1$ invariants equal to $q_{i}$.

8.2. Main theorems of Nebelung's thesis. Brigitte Nebelung completed her thesis [36, 37] in 1989 under supervision of Wolfram Jehne at Cologne. She had been introduced to computational group theory by Charles R. Leedham-Green and Joachim Neubüser. Furthermore, she had studied the details concerning 3-groups of coclass 2 with two generators in Judith A. Ascione's thesis 4, written under supervision of Mike F. Newman.

Nebelung determined explicit parametrized presentations for all isomorphism classes of metabelian 3 -groups with abelianization of type $(3,3)$ and arbitrary coclass greater than or equal to 2 , thereby extending Blackburn's results for coclass 1 in 9 . The lower and upper central series of all these groups were shown to have 3-elementary cyclic or bicyclic factors, the number of the latter being equal to the coclass. For all members of the lower central series, in particular for the abelian derived subgroup, the structure was given by abelian type invariants. Based on a computer calculation of a complete and irredundant set of isomorphism classes, listed in volume 2 [37. of her thesis, Nebelung proved that the metabelian coclass trees and their branches are arranged in periodic patterns. Finally, the number theoretic capitulation problem was solved for arbitrary number fields with 3 -class group of type $(3,3)$ by calculating the transfer kernels of any metabelian 3-group having abelianization of type $(3,3)$ with respect to its maximal subgroups.

Throughout the sequel, let $4 \leq m \leq n$ be integers and $G$ be a metabelian 3-group of order $|G|=3^{n}$ and class $\operatorname{cl}(G)=m-1$ with derived quotient $G / G^{\prime} \simeq(3,3)$.

Nebelung's starting point for analyzing the structure of the lower and upper central series of $G$ was the following inconspicuous result which proves to be very powerful. Observe that Nebelung denotes the members $\gamma_{j}(G)$ of the lower central series by $G_{j}$, for $j \geq 1$.

Theorem 8.2. (see [36, Satz 3.1.11, p. 57]) There exist generators $x, y$ of $G=\langle x, y\rangle$ such that $\gamma_{3}(G)=\left\langle x^{3}, y^{3}, \gamma_{4}(G)\right\rangle$.

Among the generators of maximal subgroups $M_{i}<G$ modulo $G^{\prime}, x$ and $y$ are indeed distinguished, in comparison to $x y$ and $x y^{-1}$, provided the class of $G$ is not too small. In the lattice of normal subgroups of $G$, the third powers $x^{3}$ and $y^{3}$ are lying near the top, namely in $\gamma_{3}(G) \backslash \gamma_{4}(G)$, whereas $(x y)^{3}$ and $\left(x y^{-1}\right)^{3}$ are lying near the bottom, namely in the second centre $\zeta_{2}(G)$, and for groups with defect $k(G)=0$ even in the centre $\zeta_{1}(G)$ of $G$, as we shall see in Theorem 8.7 .

In the following results concerning the central series of a metabelian 3-group $G$ with $G / G^{\prime}$ of type $(3,3)$, Nebelung's usage of the invariants $e$ and $s$ coincides with ours in $\S$ [3.2, but she denotes our two-step centralizers $\chi_{j}(G)$ by $C_{j}$, for $j \geq 1$. We recall that the coclass of $G$ in dependence on the invariant $e \geq 3$ is given by $\operatorname{cc}(G)=e-1 \geq 2$.

Theorem 8.3. (see [36, Satz 3.3.7, p. 70])

(1) The single-step factors of the lower central series of $G$ are given by

$$
\gamma_{j}(G) / \gamma_{j+1}(G) \simeq \begin{cases}(3,3) & \text { for } j=1 \text { and } 3 \leq j \leq e \\ (3) & \text { for } j=2 \text { and } e+1 \leq j \leq m-1 .\end{cases}
$$

(2) Consequently, the exponent $n$ of the order $|G|=3^{n}$ is related to the index of nilpotency $m$ by the inequalities $m \leq n \leq 2 m-3$ and the invariant $e$ is given by $e=n+2-m$.

(3) The two-step factors $\gamma_{j}(G) / \gamma_{j+2}(G)$ of the lower central series of $G$ are 3-elementary abelian, since $\gamma_{j}(G)^{3} \leq \gamma_{j+2}(G)$, for $j \geq 1$. 
There are two extreme cases of the relations $4 \leq m \leq n \leq 2 m-3$. For the smallest possible exponent of the order, $n=m$, with given class $m-1$, we have $e=2$ and $G$ is a CF-group of coclass $\operatorname{cc}(G)=1$ with cyclic factors $\gamma_{j}(G) / \gamma_{j+1}(G)$, except $G / G^{\prime}$. For the biggest possible exponent of the order, $n=2 m-3$, with given class $m-1$, we have $e=m-1$ and $G$ is a BF-group of coclass $\operatorname{cc}(G)=m-2 \geq 2$ with bicyclic factors $\gamma_{j}(G) / \gamma_{j+1}$, except $G^{\prime} / \gamma_{3}(G)$. BF-groups play the role of interface groups at the border between different coclass graphs in [34, $\S 3.3 .5$, Dfn. 3.3 and Thm. 3.11]. All groups, including the extreme cases, are BCF-groups with bicyclic or cyclic factors. (Nebelung uses the terminology ZEF-groups, which means Rang Zwei oder Eins Faktoren.)

From now on, we exclude groups of coclass $\operatorname{cc}(G)=1$, and we permanently rely on the following assumptions without repeating them explicitly. Let $G$ be a metabelian 3-group with commutator factor group $G / G^{\prime}$ of type $(3,3)$. Assume that $G$ has order $|G|=3^{n}$, class $\operatorname{cl}(G)=m-1$, and invariant $e=n+2-m \geq 3$, where $4 \leq m<n \leq 2 m-3$. Let generators $x, y$ of $G=\langle x, y\rangle$ be selected such that $\gamma_{3}(G)=\left\langle x^{3}, y^{3}, \gamma_{4}(G)\right\rangle, x \in G \backslash \chi_{s}(G)$ if $s<m-1$, and $y \in \chi_{s}(G) \backslash G^{\prime}$. (Nebelung calls such a couple $(x, y)$ an admissible pair of normal generators.) Let commutators of $G$ be declared by $s_{2}=t_{2}=[y, x] \in \gamma_{2}(G)$ and recursively by $s_{j}=\left[s_{j-1}, x\right], t_{j}=\left[t_{j-1}, y\right] \in \gamma_{j}(G)$ for $j \geq 3$. Starting with the powers $\sigma_{3}=y^{3}, \tau_{3}=x^{3} \in \gamma_{3}(G)$, let $\sigma_{j}=\left[\sigma_{j-1}, x\right], \tau_{j}=\left[\tau_{j-1}, y\right] \in \gamma_{j}(G)$ for $j \geq 4$.

Theorem 8.4. (see [36, Satz 3.3.7, p. 70])

(1) The two-step centralizers of the lower central series of $G$ are usually given by

$$
\chi_{j}(G)= \begin{cases}G^{\prime} & \text { for } 1 \leq j \leq e-1, \\ \left\langle y, G^{\prime}\right\rangle & \text { for } e \leq j \leq m-2, \\ G & \text { for } j \geq m-1\end{cases}
$$

except in the special case that $e=m-2$ and $\left[\chi_{s}(G), \gamma_{e}(G)\right]=\gamma_{m-1}(G)$, where

$$
\chi_{j}(G)= \begin{cases}G^{\prime} & \text { for } 1 \leq j \leq m-2, \\ G & \text { for } j \geq m-1 .\end{cases}
$$

(2) For the invariants $s$ and $e$ it follows that usually $s=e$, except in the case $e=m-2$, $\left[\chi_{s}(G), \gamma_{e}(G)\right]=\gamma_{m-1}(G)$, where $s=m-1>e$.

Let the upper central series of $G$ be defined recursively by $\zeta_{0}(G)=1$ and $\zeta_{j}(G) / \zeta_{j-1}(G)=$ Centre $\left(G / \zeta_{j-1}(G)\right)$, for $j \geq 1$. In particular, $\zeta_{1}(G)$ is the usual centre of $G$. Nebelung denotes $\zeta_{j}(G)$ by $Z_{j}$. The following theorem shows that the bicyclic factors of the upper central series of $G$ are located near the bottom of $G$, whereas the bicyclic factors of the lower central series of $G$ appear in a position near the top of $G$, by Theorem 8.3 .

Theorem 8.5. (see [36, Satz 3.3.18, p. 82])

(1) If $\left[\chi_{s}(G), \gamma_{e}(G)\right]=1$, then the factors of the upper central series of $G$ are given by

$$
\zeta_{j}(G) / \zeta_{j-1}(G) \simeq \begin{cases}(3,3) & \text { for } 1 \leq j \leq e-2 \text { and } j=m-1 \\ (3) & \text { for } e-1 \leq j \leq m-2 .\end{cases}
$$

In particular, the centre $\zeta_{1}(G)=\left\langle\sigma_{m-1}\right\rangle \times\left\langle\tau_{e}\right\rangle$ of $G$ is bicyclic of type $(3,3)$.

(2) If $\left[\chi_{s}(G), \gamma_{e}(G)\right]=\gamma_{m-1}(G)$, then the factors of the upper central series of $G$ are given by

$$
\zeta_{j}(G) / \zeta_{j-1}(G) \simeq \begin{cases}(3,3) & \text { for } 2 \leq j \leq e-1 \text { and } j=m-1 \\ (3) & \text { for } j=1 \text { and } e \leq j \leq m-2 .\end{cases}
$$

In particular, the centre $\zeta_{1}(G)=\left\langle\sigma_{m-1}\right\rangle$ of $G$ is cyclic of order 3 .

The following main theorem gives parametrized presentations for all metabelian 3-groups $G$ of coclass $\operatorname{cc}(G) \geq 2$ having abelianization $G / G^{\prime}$ of type $(3,3)$. 
Theorem 8.6. (see [36, Thm. 3.4.5, p. 94]) There exists a unique matrix $\left(\begin{array}{ll}\alpha & \beta \\ \gamma & \delta\end{array}\right)$ with entries in the finite field $\mathbb{F}_{3}$, and a unique element $\rho \in \mathbb{F}_{3}$, such that the following relations are satisfied

$$
\begin{aligned}
t_{3}^{-1} \tau_{3} \tau_{4} & =\sigma_{m-1}^{\alpha} \sigma_{m-2}^{\rho \delta} \tau_{e}^{\beta}, \\
s_{3} \sigma_{3} \sigma_{4} & =\sigma_{m-1}^{\gamma} \sigma_{m-2}^{\rho \beta} \tau_{e}^{\delta}, \\
\tau_{e+1} & =\sigma_{m-1}^{-\rho}, \\
{\left[s_{3}, y\right]=\left[t_{3}, x\right] } & =\sigma_{m-1}^{-\rho \delta}, \\
s_{2}^{3}\left(s_{3} t_{3}\right)^{3} s_{4} t_{4} & =\sigma_{m-1}^{\rho \beta}, \\
s_{2}^{3} & =\sigma_{4} \sigma_{m-1}^{-\rho \beta} \tau_{4}^{-1} .
\end{aligned}
$$

In the first two relations, put $\sigma_{m-2}=1$ in the case of $m=4$.

Concerning the parameter $\rho$, let $\left[\chi_{s}(G), \gamma_{e}(G)\right]=\gamma_{m-k}(G)$ with $0 \leq k \leq 1$. Then the defect of $G$ is $k=k(G)=0$ if and only if $\rho=0$. In particular, a group $G$ with $e=m-1$ must have $\rho=0$. The parameters of a group $G$ of coclass $\operatorname{cc}(G)=2$ are subject to various constraints:

(1) If $m=4, n=5$, then $\rho=0$ and $\left(\begin{array}{cc}\alpha & \beta-1 \\ \gamma-1 & \delta\end{array}\right) \in \mathrm{GL}_{2}\left(\mathbb{F}_{3}\right)$.

(2) If $m=5, n=6$, then $\left(\begin{array}{cc}\rho \delta & \beta-1 \\ \rho \beta-1 & \delta\end{array}\right) \in \mathrm{GL}_{2}\left(\mathbb{F}_{3}\right)$.

(3) If $5<m=n-1$, then $\beta-1 \in \mathbb{F}_{3} \backslash\{0\}$.

Consequently, a group $G$ which satisfies the assumptions of the preceding theorem is exactly the representative $G_{\rho}^{m, n}(\alpha, \beta, \gamma, \delta)$ of an isomorphism class of metabelian 3-groups $G$ with $G / G^{\prime}$ of type $(3,3)$, which satisfies the relations (13) with a fixed system of parameters $-1 \leq \alpha, \beta, \gamma, \delta, \rho \leq 1$.

By means of a computer search for a complete and irredundant set of isomorphism classes of metabelian 3-groups $G$ with $G / G^{\prime}$ of type $(3,3)$, Nebelung successively determined all admissible families $(\alpha, \beta, \gamma, \delta, \rho)$ of parameters in the relations for groups of fixed coclass $e-1$ and class $m-1$, letting the invariant $e \geq 3$ increase independently and incrementing the index of nilpotency $m \geq e+1$ in dependence on each fixed value of $e$. This revealed a double periodicity with respect to both, coclass and class, of primitive length 6 resp. 2. Consequently, it was possible to list the representatives $G_{\rho}^{m, n}(\alpha, \beta, \gamma, \delta)$ of isomorphism classes in volume 2 [37] of Nebelung's thesis in the form of finitely many parametrized presentations of infinite periodic coclass sequences where only $m$ and $n=e+m-2$ vary indefinitely for fixed coclass $e-1$. Using the periodicity, Nebelung proved that the metabelian coclass trees and their branches are arranged in periodic patterns.

Theorem 8.7. (see [36, Lem. 3.4.11, p. 105]) The third powers of $x y$ and $x y^{-1}$ are given by

$$
(x y)^{3}=\sigma_{m-2}^{\rho(\beta+\delta)} \sigma_{m-1}^{\alpha+\gamma+\rho(\beta+\delta)} \tau_{e}^{\beta+\delta}
$$

and

$$
\left(x y^{-1}\right)^{3}=\sigma_{m-2}^{\rho(\delta-\beta)} \sigma_{m-1}^{\alpha-\gamma+\rho \beta} \tau_{e}^{\beta-\delta} .
$$

In contrast to the powers $y^{3}=\sigma_{3}$ and $x^{3}=\tau_{3}$, which are elements of $\gamma_{3}(G) \backslash \gamma_{4}(G)$, the powers $(x y)^{3}$ and $\left(x y^{-1}\right)^{3}$ are contained in the second centre $\left\langle\sigma_{m-1}\right\rangle \times\left\langle\sigma_{m-2}\right\rangle \times\left\langle\tau_{e}\right\rangle=\zeta_{2}(G)$ and for $\rho=0$ even in the centre $\left\langle\sigma_{m-1}\right\rangle \times\left\langle\tau_{e}\right\rangle=\zeta_{1}(G)$. However, a uniform warranty that $(x y)^{3},\left(x y^{-1}\right)^{3} \in$ $\gamma_{4}(G)$ can only be given for a group $G$ of coclass $\operatorname{cc}(G) \geq 3$.

Theorem 8.8. (see [36, Satz 4.2.4, p. 131]) The structure of the abelian commutator subgroup $G^{\prime}$ of $G$ is given by the following direct product of nearly homocyclic abelian 3-groups.

$$
G^{\prime}= \begin{cases}\mathrm{A}(3, m-3) \times \mathrm{A}(3, m-3) & \text { in the irregular case, } \\ \mathrm{A}(3, m-2) \times \mathrm{A}(3, e-2) & \text { otherwise }\end{cases}
$$

The irregular case is characterized by $n=2 m-4, e=m-2, m \equiv 1 \bmod 2$, and

$$
\begin{cases}0 \neq \rho=\beta-1 & \text { for } m=5 \\ \rho=-1 & \text { for } m \geq 7\end{cases}
$$


Naglergasse 53, 8010 Graz, Austria

E-mail address: algebraic.number. theory@algebra.at

$U R L$ : http: //www . algebra.at 